\title{
Estimação de efeitos variantes no tempo em modelos tipo Cox via bases de Fourier e ondaletas Haar
}

\author{
Vinicius Fernando Calsavara
}

TESE APRESENTADA

AO

InSTITUTO DE MATEMÁTICA E EsTATÍSTICA

DA

UNIVERSIDADE DE SÃo PAULO

PARA

OBTENÇÃO DO TÍTULO

DE

DOUTOR EM CIÊNCIAS

\author{
Programa: Estatística \\ Orientador: Prof. Dr. Antonio Carlos Pedroso de Lima \\ Coorientadora: Prof $^{\mathrm{a}}$. Dr ${ }^{\mathrm{a}}$. Airlane Pereira Alencar
}

Durante o desenvolvimento deste trabalho o autor recebeu auxílio financeiro da CAPES

São Paulo, maio de 2015 


\title{
Estimação de efeitos variantes no tempo em modelos tipo Cox via bases de Fourier e ondaletas Haar
}

\author{
Este exemplar corresponde à redação final \\ da tese devidamente corrigida e \\ defendida por Vinicius Fernando Calsavara, \\ e aprovada pela Comissão Julgadora.
}

Comissão Julgadora:

- Prof. Dr. Antonio Carlo Pedroso de Lima (orientador) - IME-USP

- Prof $^{\mathrm{a}}$. Dr ${ }^{\mathrm{a}}$. Airlane Pereira Alencar (coorientadora) - IME-USP

- Prof. Dr. Mário de Castro Andrade Filho - ICMC-USP

- Prof. Dr. Antônio Eduardo Gomes - UNB

- Prof. Dr. João Ricardo Sato - UFABC 
"The mind, once expanded to the dimensions of larger ideas, never returns to its original size." (Oliver Wendell Holmes) 
Aos meus pais, Olga e Adauto e à minha irmã Vanessa. 


\section{Agradecimentos}

Agradeço meus orientadores, Antonio Carlos Pedroso de Lima e Airlane Pereira Alencar, pelos ensinamentos, atenção e dedicação durante todo esse período no IME-USP. Sou imensamente grato por todas as oportunidades de crescimento profissional proporcionados por eles.

Ao Prof. Mário de Castro, do ICMC-USP, pelas valiosas sugestões em minha qualificação.

Aos membros da banca examinadora, pelas contribuições e sugestões durante a defesa.

Aos amigos que fiz no IME-USP, pelas discussões acadêmcias e pelos momentos de descontração durante o desenvolvimento deste trabalho.

À Karen Rosa, pelo companheirismo e cumplicidade ao longo de todos estes anos.

Agradeço a CAPES e IME-USP pelo suporte financeiro para a realização deste trabalho.

Um agradecimento especial ao meus pais, Olga e Adauto, por terem me dado todo o apoio para que eu pudesse concluir mais uma etapa de minha formação. A vocês que, muitas vezes, renunciaram aos seus sonhos para que eu pudesse realizar o meu, partilho a alegria deste momento.

Vinicius Fernando Calsavara São Paulo, Julho de 2015. 


\section{Resumo}

O modelo semiparamétrico de Cox é frequentemente utilizado na modelagem de dados de sobrevivência, pois é um modelo muito flexível e permite avaliar o efeito das covariáveis sobre a taxa de falha. Uma das principais vantagens é a fácil interpretação, de modo que a razão de riscos de dois indivíduos não varia ao longo do tempo. No entanto, em algumas situações a proporcionalidade dos riscos para uma dada covariável pode não ser válida e, neste caso, uma abordagem que não dependa de tal suposição é necessária. Nesta tese, propomos um modelo tipo Cox em que o efeito da covariável e a função de risco basal são representadas via bases de Fourier e ondaletas de Haar clássicas e deformadas. Propomos também um procedimento de predição da função de sobrevivência para um paciente específico. Estudos de simulações e aplicações a dados reais sugerem que nosso método pode ser uma ferramenta valiosa em situações práticas em que o efeito da covariável é dependente do tempo. Por meio destes estudos, fazemos comparações entre as duas abordagens propostas, e comparações com outra já conhecida na literatura, onde verificamos resultados satisfatórios.

Palavras-chave: Modelo de regressão de Cox, riscos proporcionais, verossimilhança parcial, ondaletas de Haar, séries de Fourier. 


\section{Abstract}

The semiparametric Cox model is often considered when modeling survival data. It is very flexible, allowing for the evaluation of covariates effects. One of its main advantages is the easy of interpretation, as long as the rate of the hazards for two individuals does not vary over time. However, this proportionality of the hazards may not be true in some practical situations and, in this case, an approach not relying on such assumption is needed. In this thesis we propose a Cox-type model that allows for time-varying covariate effects, for which the baseline hazard is based on Fourier series and wavelets on a time-frequency representation. We derive a prediction method for the survival of future patients with any specific set of covariates. Simulations and an application to a real data set suggest that our method may be a valuable tool to model data in practical situations where covariate effects vary over time. Through these studies, we make comparisons between the two approaches proposed here and comparisons with other already known in the literature, where we verify satisfactory results.

Keywords: Cox regression model, proportional hazards assumption, partial likelihood, Haar wavelets, Fourier series. 


\section{Sumário}

Abreviaturas e notações $\quad$ ix

Lista de Figuras $\quad$ xi

$\begin{array}{ll}\text { Lista de Tabelas } & \text { xiii }\end{array}$

1 Introdução $\quad 1$

1.1 Organização do Trabalho . . . . . . . . . . . . . . . . . . . 3

2 Ondaletas

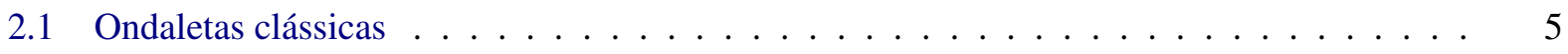

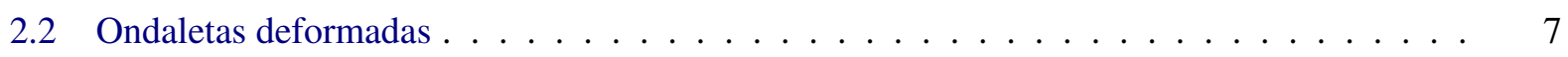

3 Modelo de regressão tipo Cox via séries de Fourier 9

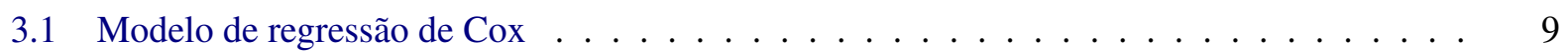

3.1.1 Estimação dos parâmetros do modelo de Cox . . . . . . . . . . . . . . . 10

3.2 Modelo tipo Cox com coeficiente dependente do tempo . . . . . . . . . . . . . . . . 11

3.3 Coeficiente dependente do tempo via séries de Fourier f . . . . . . . . . . . . . . 13

3.3.1 Estimação do efeito da covariável via séries de Fourier . . . . . . . . . . . . . . . . 14

3.3.2 Teste de hipóteses: riscos proporcionais . . . . . . . . . . . . . . . . 15

3.3 .3 Predição da função de sobrevivência . . . . . . . . . . . . . . . . . . . . 16

3.4 Estimação da função de sobrevivência via séries de Fourier . . . . . . . . . . . . . . . . . . 17

3.4 .1 Estimação dos parâmetros . . . . . . . . . . . . . . . . . . . 18

3.4.2 Testes de hipóteses: riscos proporcionais e/ou risco basal constante . . . . . . . 21

3.4 .3 Predição da função de sobrevivência . . . . . . . . . . . . . . . . . . . . 21

4 Modelo de regressão tipo Cox via ondaletas de Haar $\quad 23$

4.1 Coeficiente dependente do tempo via ondaletas de Haar . . . . . . . . . . . . . . . 23

4.1.1 Estimação do efeito da covariável via ondaletas Haar . . . . . . . . . . . . . . 25

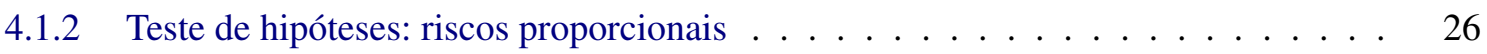

4.1 .3 Predição da função de sobrevivência . . . . . . . . . . . . . . . . . . . 26

4.2 Estimação da função de sobrevivência via ondaletas de Haar . . . . . . . . . . . . . . . 29

4.2 .1 Estimação dos parâmetros . . . . . . . . . . . . . . . . . . . . . . . . . . 29

4.2.2 Testes de hipóteses: riscos proporcionais e/ou risco basal constante . . . . . . . 32

4.2 .3 Predição da função de sobrevivência . . . . . . . . . . . . . . . . . . . . . . . 32 
5 Simulações $\quad 35$

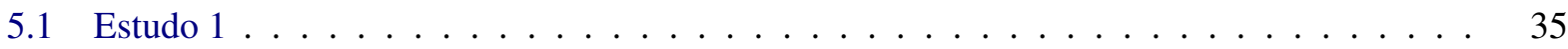

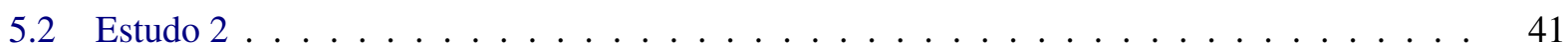

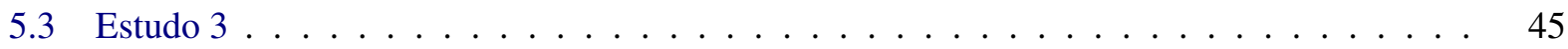

6 Aplicações $\quad 49$

6.1 Dados de cirrose biliar primária . . . . . . . . . . . . . . . . . . . . 49

6.2 Dados de câncer em estágio avançado . . . . . . . . . . . . . . . . 56

7 Considerações finais $\quad \mathbf{6 5}$

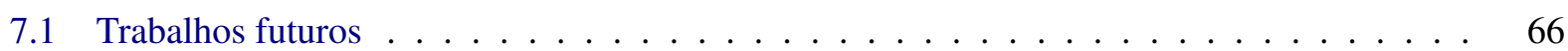

A Geração de amostras

A.1 Expansão em bases de ondaletas de Haar . . . . . . . . . . . . . . . . . . . . . . . . 69

A.2 Função contínua e crescente . . . . . . . . . . . . . . . . . . 70

B Matriz de informação observada $\quad \mathbf{7 3}$

B.1 Coeficiente dependente do tempo via séries de Fourier $\ldots \ldots \ldots$. . . . . . . . . 73

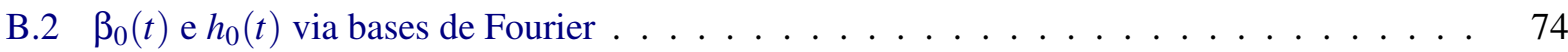

B.3 Coeficiente dependente do tempo via bases de ondaletas Haar . . . . . . . . . . . . . . 75

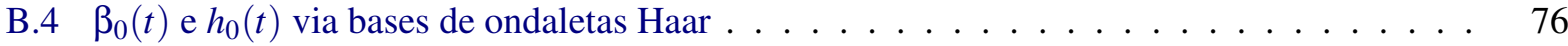

$\begin{array}{ll}\text { Referências Bibliográficas } & \mathbf{7 9}\end{array}$ 


\section{Abreviaturas e notações}

\section{Lista de Abreviaturas}

AIC Critério de informação de Akaike

BIC Critério de informação de Bayes

REQM Raiz quadrada do erro quadrático médio

\section{Lista de Símbolos}

$L_{2} \quad$ Espaço de funções mensuráveis com norma $L_{2}$ finita

$\|\cdot\|_{2} \quad$ Norma $L_{2}$

$\oplus \quad$ Soma direta entre dois espaços

$\perp \quad$ Ortogonalidade entre duas funções ou dois espaços

II Função indicadora

$\mathbb{Z} \quad$ Conjunto dos números inteiros

$\mathbb{R} \quad$ Conjunto dos números reais

$X^{\mathrm{T}} \quad$ Transposta do vetor (matriz) $X$ 


\section{Lista de Figuras}

2.1 Ondaletas de Haar. . . . . . . . . . . . . . . . . . . . . . . . . .

4.1 Estimativa do efeito da covariável ao longo do tempo (linha sólida) obtido via bases de ondaletas Haar com nível de resolução $J=2 \ldots \ldots \ldots \ldots$

5.1 Estimativa da função densidade das estimativas do coeficiente da regressão (linha contínua) e a curva teórica normal (linha pontilhada) para $n=256$ e $0 \%$ de censura. . . . . . . . .

5.2 Estimativa da função densidade das estimativas do coeficiente da regressão (linha contínua) e a curva teórica normal (linha pontilhada) para $n=256$ e $30 \%$ de censura. . . . . . . .

5.3 Estimativa da função densidade das estimativas do coeficiente da regressão (linha contínua) e a curva teórica normal (linha pontilhada) para $n=1024$ e $0 \%$ de censura. . . . . . . . .

5.4 Estimativa da função densidade das estimativas do coeficiente da regressão (linha contínua) e a curva teórica normal (linha pontilhada) para $n=1024$ e $30 \%$ de censura. . . . . . . 38

5.5 QQ plot das estimativas dos parâmetros para $n=256$ e $0 \%$ de censura. . . . . . . . . . 39

5.6 QQ plot das estimativas dos parâmetros $n=256$ e $30 \%$ de censura. . . . . . . . . . . . 39

5.7 QQ plot das estimativas dos parâmetros para $n=1024$ e $0 \%$ de censura. . . . . . . . . 40

5.8 QQ plot das estimativas dos parâmetros para $n=1024$ e $30 \%$ de censura. . . . . . . . . 40

5.9 Séries de Fourier. Estimativas médias (linha contínua) de $\beta_{0}(t)$ juntamente com a verdadeira função (linha tracejada) para $n=400$ e $30 \%$ de censura e tempos deformados. . . . . . . .

5.10 Ondaletas clássicas. Estimativas médias (linha contínua) de $\beta_{0}(t)$ juntamente com a verdadeira função (linha tracejada) para $n=400$ e $30 \%$ de censura. . . . . . . . . . . . . . .

5.11 Ondaletas deformadas. Estimativas médias (linha contínua) de $\beta_{0}(t)$ juntamente com a verdadeira função (linha tracejada) para $n=400$ e $30 \%$ de censura. . . . . . . . . . . . . .

6.1 Curva de Kaplan-Meier e intervalo de confiança pontual de $95 \%$. . . . . . . . . . . . . . 50

6.2 Estimativa de $\beta_{0}(t)$ baseado nos resíduos padronizados de Schoenfeld. . . . . . . . . . . 51

6.3 Séries de Fourier. Estimativa pontual (linha contínua) e intervalo de confiança pontual de 95\% (linha tracejada) para o efeito da variável $\log$ (protrombina) ao longo do tempo para $J=1,2$ e 3, respectivamente considerando os tempos clássicos. . . . . . . . . . . . . . .

6.4 Séries de Fourier. Estimativa pontual (linha contínua) e intervalo de confiança pontual de $95 \%$ (linha tracejada) para o efeito da variável $\log$ (protrombina) ao longo do tempo para $J=1,2$ e 3 , respectivamente considerando os tempos deformados. . . . . . . . . . .

6.5 Ondaletas clássicas. Estimativa pontual (linha contínua) e intervalo de confiança pontual de 95\% (linha tracejada) para o efeito da variável $\log$ (protrombina) ao longo do tempo para $J=0,1$ e 2 , respectivamente . . . . . . . . . . . . . . . . . . . . 
6.6 Ondaletas deformadas. Estimativa pontual (linha contínua) e intervalo de confiança pontual de $95 \%$ (linha tracejada) para o efeito da variável $\log$ (protrombina) ao longo do tempo para

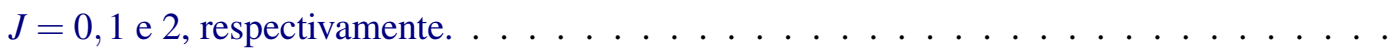

6.7 Séries de Fourier. Estimativa pontual (linha contínua) e intervalo de confiança pontual de 95\% (linha tracejada) obtido via bootstrap da função de sobrevivência para um paciente com $\log$ (protrombina $)=2,361$ estimado utilizando os tempos clássicos e deformados, res-

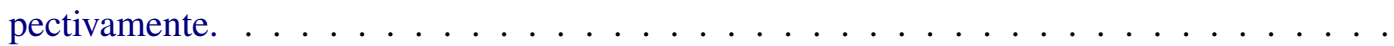

6.8 Ondaletas Haar. Estimativa pontual (linha contínua) e intervalo de confiança pontual de 95\% (linha tracejada) obtido via bootstrap da função de sobrevivência para um paciente com $\log ($ protrombina $)=2,361$ estimado utilizando as ondaletas clássicas e deformadas,

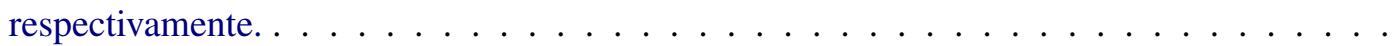

6.9 Curva de Kaplan-Meier estratificada pela covariável cirurgia juntamente com o intervalo de confiança pontual de $95 \%$. . . . . . . . . . . . . . . . . . . . 56

6.10 Estimativa de $\beta_{0}(t)$ basado nos resíduos padronizados de Schoenfeld. . . . . . . . . . . 57

6.11 Séries de Fourier via transformação clássica. Estimativa pontual (linha contínua) e intervalo de confiança pontual de $95 \%$ (linha tracejada) para o efeito da cirurgia ao longo do tempo e função de risco basal, respectivamente. . . . . . . . . . . . . . . . . . . .

6.12 Séries de Fourier via transformação deformada. Estimativa pontual (linha contínua) e intervalo de confiança pontual de $95 \%$ (linha tracejada) para o efeito da cirurgia ao longo do tempo e função de risco basal, respectivamente. . . . . . . . . . . . . . . . .

6.13 Ondaletas clássicas. Estimativa pontual (linha contínua) e intervalo de confiança pontual de 95\% (linha tracejada) para o efeito da cirurgia ao longo do tempo e função de risco basal, respectivamente. . . . . . . . . . . . . . . . . . .

6.14 Ondaletas deformadas. Estimativa pontual (linha contínua) e intervalo de confiança pontual de $95 \%$ (linha tracejada) para o efeito da cirurgia ao longo do tempo e função de risco basal, respectivamente. . . . . . . . . . . . . . . . . . .

6.15 Séries de Fourier. Estimativa pontual e intervalo de confiança pontual de 95\% (tracejada) obtido via bootstrap da função de sobrevivência condicional, clássica e deformada, respectivamente. . . . . . . . . . . . . . . . . . . . .

6.16 Ondaletas Haar. Estimativa pontual e intervalo de confiança pontual de 95\% (linha tracejada) obtido via bootstrap da função de sobrevivência condicional, clássica e deformada, respectivamente. . . . . . . . . . . . . . . . . . . . . . 


\section{Lista de Tabelas}

5.1 Resultados das simulações como função do tamanho amostral e da proporção de censura na

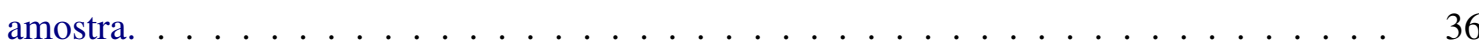

5.2 Taxas de rejeição da hipótese nula para diversos tamanhos de amostras e percentuais de

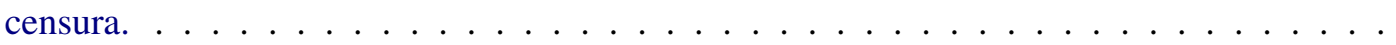

5.3 Bases de Fourier: Média amostral e desvio padrão das REQM obtidas em 100 réplicas para

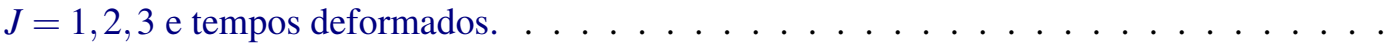

5.4 Bases de ondaletas clássicas/deformadas: Média amostral e desvio padrão das REQM obtidas em 100 réplicas para $J=0,1,2 \ldots \ldots \ldots \ldots \ldots$

5.5 Percentual do valor ótimo de $J$ selecionado pelos critérios AIC e BIC considerando as ondaletas clássicas/deformadas, respectivamente e as bases de Fourier e tempos deformados. . .

5.6 Valores médios e (desvios padrão) das 100 repetições de AIC e BIC considerando os tempos deformados. . . . . . . . . . . . . . . . . . . .

5.7 Percentual em que cada abordagem de estimação foi selecionado pelos critérios AIC e BIC considerando os tempos deformados. . . . . . . . . . . . . . . . .

5.8 Média amostral e desvio padrão das REQM obtidas pelos critérios AIC e BIC para a seleção do valor ótimo $J$ via ondaletas clássicas, deformadas e bases de Fourier, respectivamente. . $\quad 48$

6.1 Resultados do ajuste do modelo de riscos proporcionais de Cox para os dados. . . . . . . . 50

6.2 Teste da suposição de riscos proporcionais $\operatorname{com} g(t)=t \ldots \ldots \ldots \ldots$

6.3 Séries de Fourier. Estimativa dos parâmetros (erros padrão) e o critério AIC. . . . . . . . . 51

6.4 Ondaletas Haar. Estimativa dos parâmetros (erros padrão) e o critério AIC. . . . . . . . . . . 52

6.5 Testes da suposição de riscos proporcionais. . . . . . . . . . . . . . . . . . . 52

6.6 Resultados do ajuste do modelo de riscos proporcionais de Cox para os dados. . . . . . . . 57

6.7 Teste da suposição de riscos proporcionais com $g(t)=t \ldots \ldots \ldots \ldots \ldots$

6.8 Séries de Fourier. Estimativa dos parâmetros (erros padrão) e o critério AIC obtidos em duas

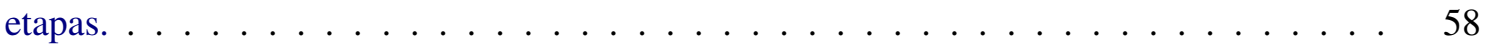

6.9 Ondaletas Haar. Estimativa dos parâmetros (erros padrão) e o critério AIC obtidos em uma

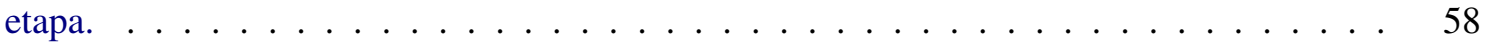

6.10 Testes de hipótese da suposição de riscos proporcionais. . . . . . . . . . . . . . . . . . . 59 


\section{Capítulo 1}

\section{Introdução}

Em estudos médicos, uma abordagem padrão para analisar dados de sobrevivência é considerar o modelo semiparamétrico de riscos proporcionais de Cox (1972), em que os coeficientes da regressão são invariantes ao longo do tempo. No entanto, há situações em que os efeitos das covariáveis podem mudar ao longo do tempo e o modelo tradicional de Cox pode ser inadequado. Na prática, usualmente ajusta-se o modelo de riscos proporcionais de Cox e avalia-se a suposição de proporcionalidade com base nos resíduos de Schoenfeld (Grambsch e Therneau, 1994; Pettitt e Bin Daud, 1990; Schoenfeld, 1982). Se forem detectados desvios da proporcionalidade dos riscos, há uma série de soluções possíveis, como a estratificação ou redefinição das covariáveis. Porém, certas aplicações práticas podem se beneficiar em assumir efeitos variantes ao longo do tempo. Por exemplo, alguns tipos de câncer podem apresentar uma boa resposta à quimioterapia em um primeiro momento, mas, devido a mecanismos genéticos, as células cancerígenas podem desenvolver alguma tolerância ao tratamento, o qual perderá o efeito ao longo do tempo. Neste caso, a estimativa direta de um parâmetro dependente do tempo pode esclarecer o processo de uma forma mais apropriada.

A fim de estimar o efeito temporal da covariável sobre a taxa de falha, diversos autores têm estudado procedimentos inferenciais em um cenário semiparamétrico, usualmente considerando o modelo de regressão de Cox com coeficientes dependentes do tempo. Zucker e Karr (1990) e Hastie e Tibshirani (1993) estudaram o problema utilizando a função de verossimilhança parcial penalizada. Gray (1992), Hess (1994), Abrahamowicz et al. (1996), Lin et al. (2006) e Saegusa et al. (2014) consideraram modelos de splines, enquanto Murphy e Sen (1991) propuseram um processo de estimação por crivo (sieve) assumindo que o coeficiente da regressão é constante por partes. Uma generalização deste método para o caso bivariado foi desenvolvida por Pedroso-de Lima e Sen (1999). Fundamentado no estimador por crivo, Murphy (1993) e Marzec e Marzec (1997) discutiram um teste para qualidade do ajuste do modelo. Martinussen et al. (2002) consideraram um procedimento de estimação iterativo para a função de coeficiente acumulado $B(t)=\int_{0}^{t} \beta_{0}(s) d s$ baseado em uma abordagem modificada da função de verossimilhança e do algoritmo iterativo de Newton-Raphson. Winnett e Sasieni (2003) propuseram estimativas não paramétricas para os coeficientes variantes no tempo com base nos resíduos de Schoenfeld derivados do modelo de riscos proporcionais (Schoenfeld, 1982). Cai e Sun (2003) consideraram uma abordagem de verossimilhança parcial local, e Tian et al. (2005) discutem um método simples em que os coeficientes dependentes do tempo são estimados via função de verossimilhança parcial, ponderado por uma função núcleo (kernel). Métodos de estimação bayesianos no modelo de Cox com efeitos dinâmicos foram desenvolvidos por Gamerman (1991) e Sargent (1997). 
Em situações práticas, quando se utiliza o modelo semiparamétrico de Cox, geralmente o maior interesse é conhecer o efeito das covariáveis sobre a taxa de falha; porém, funções relacionadas com o risco basal, $h_{0}(t)$, tais como o risco basal acumulado, $H_{0}(t)$, sobrevivência basal, $S_{0}(t)$, e principalmente, predições da função de sobrevivência para um específico indivíduo são também importantes. Na literatura há propostas para estimar a função de risco basal após o ajuste do tradicional modelo de Cox. Breslow (1972), Cox (1972) e Kalbfleisch e Prentice (1973) apresentam diferentes métodos de estimação da função $h_{0}(t)$. Cox (1972) utiliza argumentos da função de verossimilhança condicional para estimar $\beta_{0}$ e propõe estimar $h_{0}(t)$ apenas nos tempos em que ocorre a falha. Kalbfleisch e Prentice (1973) usam argumentos da função de verossimilhança marginal para estimar $\beta_{0} \mathrm{e}$, consequentemente, propõem como estimador para o risco basal uma função constante por partes, ou seja, $h_{0}(t)$ é assumida como sendo uma constante entre convenientes (mas arbitrárias) subdivisões da escala de tempo. Breslow (1972) utiliza o mesmo raciocínio, porém a subdivisão dos tempos é definida nos pontos em que ocorrem as falhas.

Em decorrência dos avanços tecnológicos, métodos não paramétricos têm ganhado cada vez mais adeptos, sendo úteis para sugerir novos modelos paramétricos e para validar outros já existentes. Por meio de métodos não paramétricos, pode-se pensar em estimar funções em vez de apenas coeficientes. Transformadas de Fourier, função núcleo e splines são algumas ferramentas matemáticas frequentemente utilizadas na estimação de funções.

Dentre as ferramentas que vêm ganhando bastante notoriedade nos últimos anos, podemos destacar as ondaletas. As bases de ondaletas costumam levar vantagem quando comparadas às bases de Fourier, visto que as ondaletas são localizadas em tempo e frequência, ao contrário das funções de Fourier, que são localizadas apenas em frequência.

Diversos trabalhos relacionados com as ondaletas vêm sendo produzidos nas mais diversas áreas. Por exemplo, as ondaletas são utilizadas em estimação de densidades (Chesneau et al., 2013; Cutillo et al. , 2013; Donoho, 1993; Hall e Patil, 1995a,b), na estimação de densidade e taxa de falha para dados censurados (Antoniadis et al., 1994, 1999), em análise de regressão não paramétrica (Donoho e Johnstone, 1995; Donoho et al., 1995, 1998; Hall e Patil, 1996; Nason e Silverman, 1994).

Embora existam alguns trabalhos envolvendo o modelo semiparamétrico de Cox com o coeficiente da regressão variando ao longo do tempo, ainda não há procedimentos de estimação de $\beta_{0}(t)$ e $h_{0}(t)$ via uma representação em bases de Fourier e ondaletas de Haar. Nesta tese, propomos estimar o efeito da covariável sobre a taxa de falha por meio de séries de Fourier e ondaletas de Haar, em um cenário semiparamétrico e, posteriormente, em um cenário totalmente paramétrico, abordamos conjuntamente a estimação da função de risco basal e do coeficiente da regressão via bases de Fourier e ondaletas Haar. Nas duas abordagens, propomos um teste para avaliar a proporcionalidade dos riscos para uma dada covariável, assim como o risco basal constante ao longo do tempo, o que é de grande interesse na prática. Além disso, um estimador da função de sobrevivência para um indivíduo específico é proposto.

Apesar de o foco principal deste trabalho ser voltado à estimação do coeficiente dependente do tempo e da função de risco basal via ondaletas de Haar, é importante mencionar que temos também o objetivo de estudar a estimação dessas funções por meio de séries de Fourier. 


\subsection{Organização do Trabalho}

Uma vez que as ondaletas possuem um papel importante neste trabalho, apresentamos resumidamente os principais conceitos de ondaletas no Capítulo 2.

No Capítulo 3, apresentamos o modelo semiparamétrico de Cox, bem como a estimação dos parâmetros do modelo. Discutimos o caso em que a suposição de riscos proporcionais é violada e o efeito variante ao longo do tempo é considerado. Expomos brevemente os principais resultados de séries de Fourier para, em seguida, apresentar o método de estimação do coeficiente dependente do tempo e também da função de risco basal. Discorremos a respeito de testes de hipóteses com o objetivo de avaliar a proporcionalidade dos riscos para uma dada covariável, assim como para a função de risco basal constante ao longo do tempo. Além disso, um método de predição da função de sobrevivência para um indivíduo específico é derivado.

Considerando as bases de ondaletas de Haar, propomos no Capítulo 4, um modelo tipo Cox em que o coeficiente da regressão e o risco basal são representados por séries de ondaletas de Haar. Por meio de testes de hipóteses, propomos um método de verificação de riscos proporcionais para uma dada covariável, bem como risco basal constante ao longo do tempo. Para um indivíduo específico, propomos ainda um método de predição da função de sobrevivência.

Finalizando o trabalho, apresentamos no Capítulo 7 algumas conclusões e comentários sobre pontos que não foram mencionados no decorrer do texto. Além disso, no Apêndice A apresentamos o procedimento de geração de amostras utilizados nos estudos de simulação. Os elementos da matriz de informação observada são apresentados no Apêndice B. 


\section{Capítulo 2}

\section{Ondaletas}

Resumimos neste capítulo os conceitos fundamentais de ondaletas que serão necessários nas seções posteriores. Mais detalhes da teoria de ondaletas podem ser encontrados em Daubechies (1992), Härdle et al. (1998), Mallat (2008), Morettin (1999), Nason (2008) e Vidakovic (1999).

\subsection{Ondaletas clássicas}

A análise de Fourier, a análise utilizando splines e ondaletas consistem na expansão ou na aproximação de certas funções usando, respectivamente, senos e cossenos, polinômios e ondaletas. Séries de ondaletas costumam levar vantagem quando comparadas às séries de Fourier, visto que elas são, simultaneamente, localizadas em tempo e frequência, ao contrário das séries de Fourier, que são localizadas apenas em frequência.

$\mathrm{Na}$ literatura, diversos trabalhos estatísticos relacionados com as ondaletas vêm sendo produzidos, nas mais diversas áreas. Por exemplo, elas são utilizadas na estimação de densidades (Chesneau et al. , 2013; Cutillo et al., 2013; Donoho, 1993; Hall e Patil, 1995a,b), na estimação de densidade e taxa de falha para dados censurados (Antoniadis et al., 1994, 1999) e na análise de regressão não paramétrica (Donoho e Johnstone, 1995; Donoho et al., 1995, 1998; Hall e Patil, 1996; Nason e Silverman, 1994), entre outras áreas. Devido à sua analogia com as séries de Fourier, considere o espaço $L_{2}(\mathbb{R})$ de todas as funções mensuráveis quadrado integrável sobre $\mathbb{R}$. Assim, se $\beta_{0} \in L_{2}(\mathbb{R})$, então

$$
\left\|\beta_{0}\right\|_{2}=\left(\int_{\mathbb{R}}\left|\beta_{0}(t)\right|^{2} d t\right)^{1 / 2}<\infty
$$

Uma base $\left\{\phi_{j, k}(t) \cup \psi_{j, k}(t), j, k \in \mathbb{Z}\right\}$ do espaço $L_{2}(\mathbb{R})$ pode ser gerada por meio de uma dilatação binária e de uma translação diádica via uma ondaleta pai $\phi$ e uma ondaleta mãe $\psi$, em que

$$
\phi_{j, k}(t)=2^{j / 2} \phi\left(2^{j} t-k\right) \quad \text { e } \quad \psi_{j, k}(t)=2^{j / 2} \psi\left(2^{j} t-k\right), \quad j, k \in \mathbb{Z} .
$$

A ortonormalidade dessa base depende da escolha das ondaletas $\phi$ e $\psi$. Se a ondaleta tiver suporte compacto no intervalo $[0, N]$, então $\phi_{j, k}\left(\right.$ ou $\left.\psi_{j, k}\right)$ terá suporte em $\left[2^{-j} k, 2^{-j}(N+k)\right]$.

A função $\phi$ deve satisfazer $\int_{\mathbb{R}} \phi=1$, enquanto a função $\psi$ deve ser tal que $\int_{\mathbb{R}} \psi=0$ (admissível) e $\int_{\mathbb{R}}|\psi|<\infty$. Uma ondaleta é dita $r$-regular se possuir $r$ momentos não nulos e $r$ derivadas contínuas.

Uma base de ondaletas tem associada uma análise de multirresolução em $L_{2}(\mathbb{R})$, o que permite a análise dos dados por distintos níveis de resolução, adicionando ou desprezando detalhes. Uma análise 
de multirresolução corresponde a uma sequência de espaços fechados, os quais são encaixados, e crescem para $L_{2}(\mathbb{R})$, quando $j \rightarrow \infty$. Sejam $V_{j}$ e $W_{j}$ os subespaços fechados gerados por $\left\{\phi_{j, k}, k=1, \ldots, 2^{j}\right\}$ e $\left\{\psi_{j, k}, k=1, \ldots, 2^{j}\right\}$, respectivamente. Então, a análise de multirresolução associada satisfaz, para todo $j \in \mathbb{Z}:$

1) $V_{j} \subset V_{j+1}$,

2) $\bigcup_{j} V_{j}$ é denso em $L_{2}(\mathbb{R})$

3) $\bigcap_{j} V_{j}=\emptyset \mathrm{e}$

4) $V_{j+1}=V_{j} \oplus W_{j}, \quad W_{j} \perp V_{j}$.

Assim, a partir de um certo espaço de multirresolução de nível $j_{0}$, qualquer função $\beta_{0} \in L_{2}(\mathbb{R})$ pode ser expandida em uma série de ondaletas de tal modo que

$$
\beta_{0}(t)=\sum_{k} c_{j_{0}, k} \phi_{j_{0}, k}(t)+\sum_{j \geq j_{0}} \sum_{k} d_{j, k} \psi_{j, k}(t)
$$

Essa transformada de ondaletas decompõe uma função em componentes com diferentes resoluções. Os coeficientes do nível mais grosso representados pelos termos $c_{j_{0}, k}$, captam a estrutura grossa da função (parte suave da função), enquanto os coeficientes $d_{j, k}$, representam as estruturas mais finas (detalhes da função) à medida em que o nível de resolução $j$ aumenta.

O exemplo mais simples e antigo de ondaleta é a função de Haar (Haar, 1910; Shumway e Stoffer, 2010), em que

$$
\phi(t)=\left\{\begin{array}{lc}
1, & \text { se } 0 \leq t<1, \\
0, & \text { caso contrário, }
\end{array} \quad \text { e } \psi(t)=\left\{\begin{array}{rrr}
1, & \text { se } 0 \leq t<0,5, \\
-1, & \text { se } 0,5 \leq t<1, \\
0, & \text { caso contrário. }
\end{array}\right.\right.
$$

A partir dessas funções é possível obter as ondaletas $\phi_{j, k}(\cdot)$ e $\psi_{j, k}(\cdot)$, que dependem do nível de resolução $j$ e da escala $k$. Vale destacar que as funções $\phi_{0,0}(\cdot)$ e $\psi_{0,0}(\cdot)$ são equivalentes às ondaletas pai e mãe, respectivamente, as quais podem ser visualizadas na Figura 2.1. Apresentamos ainda nessa figura as ondaletas $\psi_{1,0}(\cdot)$ e $\psi_{1,1}(\cdot)$.

Alguns exemplos de ondaletas bastante conhecidas são daublets (ou Daubechies), symmlets, coiflets, ondaletas de Meyer e ondaletas de Shannon. Vale destacar que as ondaletas de Haar têm suporte compacto no intervalo unitário, pertencem à classe das daublets e possuem forma analítica, o que as torna bem interessantes.

Daqui em diante, consideramos apenas a base de ondaletas de Haar, em que o nível de escala mais baixo é fixado em $j_{0}=0$, de tal modo que a função (2.1) é reescrita como

$$
\beta_{0}(t)=c \phi_{0,0}(t)+\sum_{j=0}^{J} \sum_{k=0}^{2^{j}-1} d_{j, k} \psi_{j, k}(t)
$$

em que $J$ representa o nível de resolução, enquanto $c, d_{0,0}, \ldots, d_{J, 2^{J}-1}$ são os coeficientes a serem estimados. Vale destacar que o número de parâmetros dobra com o incremento em uma unidade no nível de resolução $J$. Além disso, as ondaletas $\phi_{0,0}(\cdot)$ e $\left\{\psi_{j, k}(\cdot), j=0, \ldots, J ; k=0, \ldots, 2^{J}-1\right\}$ são não nulas apenas no intervalo $[0,1)$, tornando necessário redimensionar os tempos observados para esse intervalo quando utilizamos as ondaletas clássicas. 

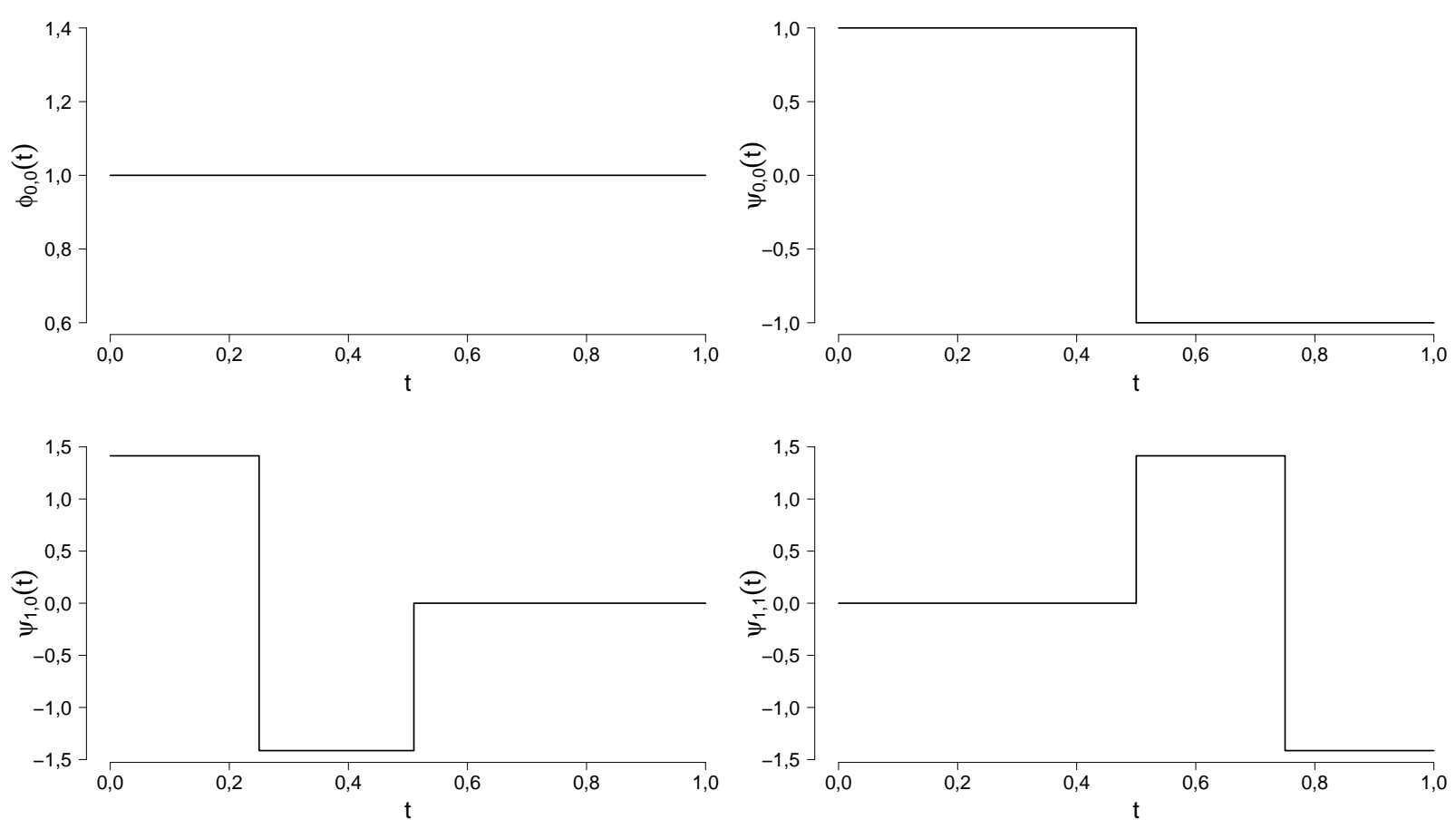

Figura 2.1: Ondaletas de Haar.

A principal vantagem em adotar apenas a base de ondaleta de Haar está associada ao fato de a função $\phi_{0,0}(t)$ ser constante no intervalo $[0,1)$, o que possibilita a função $\beta_{0}(t)$ ser também constante, caso os coeficientes de detalhes $d_{j, k}$ sejam todos nulos, o que é um dos principais objetivos deste trabalho.

É importante ainda ressaltar que adotar outras bases de ondaletas inviabiliza, por meio de testes de hipóteses, avaliar se a função $\beta_{0}(t)$ é constante ao longo do tempo. Isso ocorre, pois a função de Haar é a única base de ondaletas a apresentar $\phi(t)$ constante.

\subsection{Ondaletas deformadas}

Apesar de suas vantagens consideráveis, as ondaletas clássicas podem apresentar limitações no contexto de análise de sobrevivência. $\mathrm{O}$ uso das ondaletas tradicionais costuma ser baseado em suposições como delineamento fixo e uniforme dos dados. Tal suposição se faz necessária devido ao uso frequente das transformadas discretas de ondaletas, pois estas requerem que as funções a serem analisadas sejam avaliadas em instantes igualmente espaçados (Daubechies, 1992). Por exemplo, em regressão não paramétrica os procedimentos de estimação são restritos a amostras com observações equidistantes (Cai e Brown, 1998). No entanto, em situações práticas, como em dados de sobrevivência, é comum os tempos de vida serem desigualmente espaçados e usualmente com poucas observações completas no final do experimento, acarretando, em geral, maiores amplitudes dos intervalos de confiança pontuais de $\beta_{0}(t)$ nos tempos finais. Como alternativa às ondaletas clássicas, apresentamos os conceitos de ondaletas deformadas (Cai e Brown, 1998; Kerkyacharian e Picard, 2004).

A fim de descrever brevemente sobre ondaletas deformadas, sejam $T_{1}, \ldots, T_{n}$ variáveis aleatórias independentes e identicamente distribuídas representando os tempos de vida e considere $F$ alguma função distribuição no intervalo $[0,1]$. Sejam $T_{(1)}<\cdots<T_{(n)}$ os tempos ordenados. Para uma certa função $F$ inver- 
sível (com função inversa $F^{-1}$ ), considere

$$
T_{(i)}=F^{-1}(i / n), \quad i=1, \ldots, n
$$

Utilizando o fato de que

$$
\beta_{0}\left(T_{(i)}\right)=\beta_{0}\left(F^{-1}(i / n)\right)
$$

a expansão (2.3) é utilizada para estimar (2.4) via ondaletas de Haar.

Neste trabalho, a função $F$ é dada pela função distribuição empírica, $\widehat{F}$, a qual é escrita na forma

$$
\widehat{F}(t)=\frac{1}{n} \sum_{i=1}^{n} \mathbb{I}_{\left\{T_{i} \leq t\right\}}
$$

em que II corresponde à função indicadora, sendo que esta é igual a 1 , se $T_{i} \leq t$, e 0 , caso contrário.

Denotemos $\beta_{0} \circ F^{-1}$ por $\dot{g}$. A função $\dot{g}$ pode ser representada por uma base de ondaletas como em (2.3), de modo que

$$
\dot{g}(y)=c \phi_{0,0}(y)+\sum_{j=0}^{J} \sum_{k=0}^{2^{j}-1} d_{j, k} \psi_{j, k}(y),
$$

em que $y \in[0,1]$. Considere $y=F(t)$, para qualquer $t \geq 0$, logo

$$
\beta_{0}(t)=c \phi_{0,0}(F(t))+\sum_{j=0}^{J} \sum_{k=0}^{2^{j}-1} d_{j, k} \psi_{j, k}(F(t)) .
$$

A base utilizada na análise de $\beta_{0}$ não é mais ortonormal, sendo que as ondaletas utilizadas passam a ser “deformadas" pela função $F$, de onde surge o nome que define essa classe de ondaletas. Por outro lado, há algumas vantagens em utilizar as ondaletas deformadas, dentre elas, destacamos o fato de o método tratar os tempos em instantes igualmente espaçados no intervalo unitário. 


\section{Capítulo 3}

\section{Modelo de regressão tipo Cox via séries de Fourier}

Neste capítulo, discutimos o modelo de regressão de riscos proporcionais proposto por Cox (1972), a fim de modelar uma possível relação entre o tempo de sobrevivência e um vetor de covariáveis. Essa abordagem permite estimar os parâmetros da regressão associados aos efeitos destas covariáveis, de modo que a razão entre as taxas de riscos não varia ao longo do tempo. Porém, em algumas aplicações práticas considerar o efeito da covariável ao longo do tempo pode ser importante. Deste modo, uma abordagem que não dependa da suposição de riscos proporcionais é necessária. Neste capítulo, abordamos inicialmente o problema em um cenário semiparamétrico, em que o coeficiente dependente do tempo é representado por meio das séries de Fourier. Posteriormente, consideramos as bases de Fourier na estimação conjunta do risco basal e no efeito da covariável sobre a taxa de falha.

Organizamos este capítulo da forma como segue. Na Seção 3.1, apresentamos brevemente o modelo de regressão de Cox. Na Seção 3.2, discutimos o caso em que a suposição de riscos proporcionais é violada e o efeito dependente do tempo é considerado. Abordamos na Seção 3.3 a estimação do coeficiente dependente do tempo, a qual é baseada no método de máxima verossimilhança parcial. Discutimos também, por meio de testes de hipóteses, uma forma de avaliar o pressuposto de proporcionalidade dos riscos para uma dada covariável. Além disso, propomos um estimador para a função de sobrevivência condicional. Na Seção 3.4, discutimos a estimação conjunta do risco basal e do coeficiente variante ao longo do tempo. Neste cenário, a função de risco basal é também representada em bases de Fourier e a estimação dos parâmetros é feita utilizando o método de máxima verossimilhança. Finalizando o capítulo, propomos um método de predição da função de sobrevivência para um indivíduo específico.

\subsection{Modelo de regressão de Cox}

O modelo de regressão de Cox (1972) é frequentemente utilizado para modelar uma possível relação entre o tempo de sobrevivência $T$ e um vetor de covariáveis $\mathbf{X}=\left(X_{1}, \ldots, X_{s}\right)^{\mathrm{T}} \in \mathbb{R}^{s}$. A suposição básica para o seu uso é que a razão das taxas de falhas de dois indivíduos quaisquer não varia ao longo do tempo. Considere $T^{*}$ o tempo de falha e $C$ a variável correspondente ao tempo de censura. Condicional a $\mathbf{X}$, assumimos que $T^{*}$ e $C$ são independentes. Os dados disponíveis são da forma $\left(T_{1}, \delta_{1}, \mathbf{X}_{1}\right), \ldots,\left(T_{n}, \delta_{n}, \mathbf{X}_{n}\right)$, em que $T_{i}=\min \left(T_{i}^{*}, C_{i}\right)$, e $\delta_{i}$ é igual a 1 se $T_{i}=T_{i}^{*}$, e 0 caso contrário. Seja $h(t \mid \mathbf{x})=\lim _{\Delta t \rightarrow 0}(\Delta t)^{-1} P(t \leq$ $T<t+\Delta t \mid T \geq t, \mathbf{X}=\mathbf{x})$ a função taxa de falha ou risco condicional de $T$ dado $\mathbf{X}=\mathbf{x}$. O modelo de riscos 
proporcionais de Cox assume a seguinte forma (Cox, 1972, 1975):

$$
h(t \mid \mathbf{x})=h_{0}(t) g\left(\mathbf{x}^{\mathrm{T}} \beta_{0}\right),
$$

em que $h_{0}(\cdot)$ representa a função de risco basal, supostamente arbitrária mas não negativa, para a unidade quando $\mathbf{x}=\mathbf{0}, \beta_{0}=\left(\beta_{0_{1}}, \ldots, \beta_{0_{s}}\right)^{\mathrm{T}} \in \mathbb{R}^{s}$ é um vetor de coeficientes a serem estimados e $g\left(\mathbf{x}^{\mathrm{T}} \boldsymbol{\beta}_{0}\right)$ é uma função não negativa dependente de $\mathbf{x}$.

Neste modelo, em geral, assume-se que as covariáveis têm um efeito multiplicativo sobre a taxa de falha, suposição que parece ser razoável em muitas situações. Cox e Oakes (1984) apresentam três formulações para $g\left(\mathbf{x}^{\mathrm{T}} \beta_{0}\right)$. A que se tornou comumente a mais utilizada é a forma $\exp \left(\mathbf{x}^{\mathrm{T}} \beta_{0}\right)$ garantindo valores não negativos para a taxa de falha para qualquer combinação de $\mathbf{x}$ e $\beta_{0}$.

O modelo em questão pode ser considerado semiparamétrico, pois é composto de dois componentes, um paramétrico $\left[\exp \left(\mathbf{x}^{\mathrm{T}} \beta_{0}\right)\right]$ e o outro $\left[h_{0}(\cdot)\right]$ não paramétrico. Notamos que a estrutura (3.1) também ocorre em um ambiente paramétrico, como é o caso das distribuições exponencial e Weibull, entre outras (neste caso não é referenciado como modelo de Cox, mas sim uma modificação).

\subsubsection{Estimação dos parâmetros do modelo de Cox}

Para estimar os parâmetros do modelo de riscos proporcionais de Cox, o método de máxima verossimilhança costuma ser utilizado, considerando a informação de todos os tempos observados, censurados ou não, produzindo sob certas condições de regularidade, estimativas consistentes e assintoticamente normais.

Em geral, para estimar os parâmetros do modelo de Cox, é utilizada a função de verossimilhança parcial proposta por Cox (1975) que, para estimar $\beta_{0}$, condicionou a verossimilhança, eliminando a componente $h_{0}(\cdot)$.

Considere a situação em que ocorrem $D \leq n$ falhas nos distintos tempos $t_{1}<\cdots<t_{D}$. Na ausência de empates, a função de verossimilhança parcial (Cox, 1972, 1975) sob censura não informativa é expressa por

$$
\mathcal{L}\left(\beta_{0}\right)=\prod_{i=1}^{n}\left\{\frac{\exp \left(\mathbf{x}_{i}^{\mathrm{T}} \beta_{0}\right)}{\sum_{q \in R\left(t_{i}\right)} \exp \left(\mathbf{x}_{q}^{\mathrm{T}} \beta_{0}\right)}\right\}^{\delta_{i}},
$$

em que $R\left(t_{i}\right)$ representa o conjunto de índices de todos os indivíduos da amostra que estão em risco no instante de falha $t_{i}^{-}$, ou seja, o conjunto discrimina todos os sujeitos que ainda não falharam e não foram censurados até o momento $t_{i}^{-}$.

A correspondente função logverossimilhança parcial, $\ell\left(\beta_{0}\right)=\log \mathcal{L}\left(\beta_{0}\right)$, é dada por

$$
\ell\left(\beta_{0}\right)=\sum_{i=1}^{n} \delta_{i}\left\{\mathbf{x}_{i}^{\mathrm{T}} \beta_{0}-\log \sum_{q \in R\left(t_{i}\right)} \exp \left(\mathbf{x}_{q}^{\mathrm{T}} \beta_{0}\right)\right\} .
$$

Os tempos de censura não são incorporados diretamente na função de verossimilhança parcial, mas os indivíduos censurados contribuem para $\mathcal{L}\left(\beta_{0}\right)$ através do conjunto de índices $R\left(t_{i}\right)$ somente enquanto permanecem em risco.

Os valores de $\beta_{0}$ que maximizam a função de verossimilhança parcial são obtidos resolvendo o sistema de equações

$$
\mathbf{U}\left(\beta_{0}\right)=\frac{\partial \ell\left(\beta_{0}\right)}{\partial \beta_{0}}=\sum_{i=1}^{n} \delta_{i}\left\{\mathbf{x}_{i}^{\mathrm{T}}-\frac{\sum_{q \in R\left(t_{i}\right)} x_{q} \exp \left(\mathbf{x}_{q}^{\mathrm{T}} \beta_{0}\right)}{\sum_{q \in R\left(t_{i}\right)} \exp \left(\mathbf{x}_{q}^{\mathrm{T}} \beta_{0}\right)}\right\}=\mathbf{0}
$$


em que $\mathbf{U}\left(\beta_{0}\right)$ é o vetor escore de derivadas de primeira ordem da função $\ell\left(\beta_{0}\right)$ em relação a $\beta_{0}$. A solução de $\mathbf{U}\left(\beta_{0}\right)$ pode ser encontrada numericamente por meio de métodos iterativos e geralmente, envolve o método numérico de Newton-Raphson. Vale ressaltar que (3.2) não depende do risco basal, tanto que inferências com relação aos efeitos das covariáveis sobre a taxa de falha podem ser feitas sem conhecer a forma da função $h_{0}(\cdot)$.

A função de verossimilhança parcial não pressupõe a possibilidade de empates nos valores observados, porém na prática, devido à escala de medida, empates nos tempos de falha e/ou de censura podem ocorrer. Propostas de modificação da função de verossimilhança parcial para incorporar empates foram desenvolvidas por Breslow (1972), Peto (1972), Efron (1977), Farewel e Prentice (1980), entre outros.

Motivado heuristicamente pela função de verossimilhança parcial, Cox (1972) usou sem demonstrar que a solução para as equações de estimação obtidas a partir da verossimilhança parcial fornecem estimativas com as mesmas propriedades dos estimadores baseados na verossimilhança completa, aplicando a teoria de máxima verossimilhança à verossimilhança parcial. Posteriormente, Cox (1975) e Tsiatis (1981), sob suposições específicas, mostraram que os estimadores de máxima verossimilhança parcial são consistentes e assintoticamente normais. Uma justificativa rigorosa e mais geral foi desenvolvida por Andersen e Gill (1982).

\subsection{Modelo tipo Cox com coeficiente dependente do tempo}

Muitas vezes em situações práticas, a suposição de riscos proporcionais é violada e o modelo tradicional de Cox pode ser inadequado. Usualmente, ajusta-se o modelo de riscos proporcionais de Cox e avalia-se a suposição de proporcionalidade por meio dos resíduos de Schoenfeld (Schoenfeld, 1982) e se tal violação for detectada, estratificação ou redefinição da covariável são soluções possíveis. Porém assumir que o efeito varia ao longo do tempo pode ser uma boa alternativa. Nesta situação, a estimativa direta de um parâmetro dependente do tempo pode esclarecer o processo de maneira apropriada.

A fim de obter estimativas dos coeficientes variantes ao longo do tempo, diversos autores têm estudado procedimentos inferenciais em um cenário semiparamétrico, usualmente considerando o modelo de Cox com o coeficiente da regressão dependente do tempo.

Por simplicidade, daqui em diante, consideraremos apenas uma covariável observada $(s=1)$ para cada indivíduo assumindo valor $x$, de modo que o correspondente coeficiente da regressão é assumido ser dependente do tempo. Assim, a função de risco condicional (3.1) é reescrita como

$$
h(t \mid x)=h_{0}(t) \exp \left(x \beta_{0}(t)\right)
$$

Observe que nesta formulação, este modelo leva a riscos não proporcionais, a menos que $\beta_{0}(t)$ seja constante e igual a $\beta_{0}$, para todo $t \geq 0$. No caso especial de uma variável binária, o modelo (3.4) reduz-se a

$$
h(t \mid 0)=h_{0}(t) \quad \text { e } \quad h(t \mid 1)=h_{0}(t) \exp \left(\beta_{0}(t)\right)
$$

Neste caso, $\beta_{0}(t)$ mede o logarítmo do risco relativo entre os dois grupos.

Pode-se propor, em um primeiro momento, representar o coeficiente dependente do tempo por meio de funções-núcleo, splines, bases de Fourier e ondaletas, entre outras. De um modo geral, para uma representação qualquer do efeito da covariável sobre a taxa de falha, $\beta_{0}(t)$, a função logverossimilhança parcial (3.3) 
é reescrita como

$$
\ell(\theta)=\sum_{i=1}^{n} \delta_{i}\left[x_{i} \beta_{0}\left(t_{i}\right)-\log \sum_{q \in R\left(t_{i}\right)} \exp \left(x_{q} \beta_{0}\left(t_{i}\right)\right)\right] .
$$

em que $\theta$ representa o vetor de parâmetros a ser estimado. No caso específico de uma representação em bases de ondaletas de Haar, o vetor $\theta$ será composto pelos coeficientes $c, d_{0,0}, \ldots, d_{J, 2^{J}-1}$, em que $J$ é o nível de resolução.

Por outro lado, se o risco basal é especificado, as estimativas do efeito da covariável sobre a taxa de falha e da função de risco basal são obtidas pelo método de máxima verossimilhança. Neste cenário, o vetor de parâmetros $\theta$ será composto pelos parâmetros/coeficientes da função de risco basal e do coeficiente dependente do tempo. Assim, sob censura não informativa, a função de verossimilhança (ou verossimilhança completa) de $\theta$ para estimar $\beta_{0}(t)$ e $h_{0}(t)$ (Klein e Moeschberger, 2003) é

$$
\mathcal{L}(\theta)=\prod_{i=1}^{n} h\left(t_{i} \mid x_{i}\right)^{\delta_{i}} \exp \left(-\int_{0}^{t_{i}} h\left(s \mid x_{i}\right) d s\right),
$$

e, consequentemente, a função logverossimilhança é dada por

$$
\ell(\theta)=-\sum_{i=1}^{n} \int_{0}^{t_{i}} h\left(s \mid x_{i}\right) d s+\sum_{i=1}^{n} \delta_{i} \log h_{0}\left(t_{i}\right)+\sum_{i=1}^{n} \delta_{i} x_{i} \beta_{0}\left(t_{i}\right) .
$$

É importante mencionar que dependendo da estrutura funcional da função de risco condicional, o primeiro termo do lado direito em (3.7) pode não apresentar primitiva em forma analítica, necessitando assim de métodos para integração numérica: as fórmulas de Newton-Cotes e quadratura de Gauss-Legendre (Campos Filho, 2007), método de Monte Carlo (Gamerman e Lopes, 2006), bem como outras técnicas podem ser aplicadas.

Os estimadores de máxima verossimilhança parcial e de máxima verossimilhança, $\widehat{\theta}$, são obtidos resolvendo os sistemas de equações

$$
\mathbf{U}(\theta)=\frac{\partial \ell(\theta)}{\partial \theta}=\mathbf{0}
$$

em que $\mathbf{U}(\theta)$ é o vetor escore de derivadas de primeira ordem da função $\ell(\theta)$ apresentadas em (3.5) e (3.7), respectivamente.

Em situações práticas, uma vez ajustado o modelo de regressão pode surgir o interesse em verificar a proporcionalidade dos riscos para uma dada covariável, $\beta_{0}(t)=\beta_{0}$, para todo $t \geq 0$, bem como avaliar se a função de risco basal é constante ao longo do tempo. Dependendo da estrutura funcional de $\beta_{0}(t)$ pode-se pensar em aplicar testes de hipóteses para responder a tais interesses.

Quando se utiliza o modelo de regressão de Cox ou tipo Cox, o principal objetivo está associado ao efeito da covariável sobre a taxa de falha. Porém, funções relacionadas com o risco basal, $h_{0}(\cdot)$, e consequentemente, predições da função de sobrevivência para um indivíduo específico também são importantes.

Como alternativa aos procedimentos já conhecidos na literatura, na próxima seção, propomos inicialmente expandir o coeficiente dependente do tempo por meio de séries de Fourier considerando um cenário semiparamétrico. A fim de verificar se a proporcionalidade dos riscos para uma dada covariável se sustenta, um procedimento de avaliação também é proposto. Além disso, apresentamos um método de predição da função de sobrevivência para um indivíduo específico. 


\subsection{Coeficiente dependente do tempo via séries de Fourier}

Antes de discutirmos a respeito da estimação do coeficiente dependente do tempo por meio de séries de Fourier, discorremos brevemente sobre essa importante ferramenta matemática. Mais detalhes da teoria de Fourier podem ser encontrados em Bachmann et al. (2000), Morettin (1999), Morettin e Toloi (2006) e Vidakovic (1999).

A ideia central da teoria das séries de Fourier é representar a função $\beta_{0}(t)$ dada em (3.4) por uma série infinita de funções trigonométricas, periódica e de período fundamental $L$ na forma (Vidakovic, 1999),

$$
\beta_{0}(t)=\frac{c_{0}}{2}+\sum_{k=1}^{\infty}\left\{c_{k} \cos \left(k \omega_{0} t\right)+d_{k} \sin \left(k \omega_{0} t\right)\right\}
$$

em que $\omega_{0}$ é a frequência angular fundamental de $\beta_{0}$ (e também da série de Fourier), dada por $\omega_{0}=2 \pi / L$, enquanto $c_{0}, c_{k}$ e $d_{k},\left(k \in \mathbb{Z}_{*}^{+}\right)$são chamados de coeficientes de Fourier.

De um modo geral, esta é uma das ideias básicas da análise, decompor funções arbitrárias em termos de outras mais simples (senos e cossenos), em que cada parcela da série é uma combinação linear de funções trigonométricas. Vale destacar que se a função $\beta_{0}(\cdot)$ é conhecida e devido à ortogonalidade das funções seno e cosseno, os coeficientes de Fourier são facilmente obtidos utilizando a transformada discreta de Fourier. Entretanto, em muitas situações a forma funcional de $\beta_{0}(\cdot)$ é desconhecida, o que impossibilita determinar, de forma tradicional, os coeficientes de Fourier. Nessa situação, obter estimativas dos coeficientes pode ser uma solução plausível.

Neste trabalho, supomos que o coeficiente dependente do tempo é uma função definida no intervalo $[0,1)$, periódica, com período fundamental $L=1$, de modo que a expansão em séries de Fourier (3.8) é reescrita como uma soma finita de senos e cossenos

$$
\beta_{0}(t)=\frac{c_{0}}{2}+\sum_{k=1}^{J}\left\{c_{k} \cos (2 k \pi t)+d_{k} \sin (2 k \pi t)\right\}, \quad \text { para inteiro } \quad J \geq 1 .
$$

Vale observar que o efeito da covariável sobre a taxa de falha é uma função desconhecida, o que impede, na sua forma usual, a determinação dos coeficientes de Fourier. Desse modo, os coeficientes serão tratados como parâmetros, e consequentemente, estimativas de máxima verossimilhança serão obtidas.

Para isso, considere que o coeficiente da regressão é uma função como em (3.9), de tal modo que o risco condicional (3.4) do modelo de regressão tipo Cox é

$$
h(t \mid x)=h_{0}(t) \exp \left(x\left[\frac{c_{0}}{2}+\sum_{k=1}^{J}\left\{c_{k} \cos (2 k \pi t)+d_{k} \sin (2 k \pi t)\right\}\right]\right),
$$

em que $h_{0}(\cdot)$ é a função de risco basal completamente não especificada, porém não negativa, enquanto $c_{0}$, $c_{k}, d_{k},(k=1, \ldots, J)$, são os parâmetros a serem estimados.

É importante mencionar que os valores de $J$ podem ser considerados como parâmetros de suavização e tem papel similar ao número de nós, quando se utiliza splines na estimação de funções. Neste trabalho, o valor ótimo de $J$ será obtido a partir do erro quadrático médio e também por meio dos critérios de seleção AIC e BIC.

O modelo (3.10) generaliza o modelo tradicional de Cox, pois permite riscos não proporcionais, a menos que a função $\beta_{0}(t)$ seja constante ao longo do tempo, que é o caso quando os coeficientes $c_{k}^{\prime} s$ e $d_{k}^{\prime} s$ são todos nulos. 


\subsubsection{Estimação do efeito da covariável via séries de Fourier}

A estimação dos parâmetros do modelo (3.10) é realizada por meio do método de máxima verossimilhança parcial, que é baseado na função de verossimilhança parcial (3.5). Neste cenário, o vetor de parâmetros do modelo é composto pelos coeficientes de Fourier, $\theta=\left(c_{0}, c_{1}, \ldots, c_{J}, d_{1}, \ldots, d_{J}\right)^{\mathrm{T}} \in \mathbb{R}^{p}$ de dimensão $p=2 J+1$. A função logverossimilhança parcial de $\theta$ para estimar $\beta_{0}(t)$ é

$$
\begin{aligned}
\ell(\theta)= & \sum_{i=1}^{n} \delta_{i}\left(x_{i}\left[\frac{c_{0}}{2}+\sum_{k=1}^{J}\left\{c_{k} \cos \left(2 k \pi t_{i}\right)+d_{k} \sin \left(2 k \pi t_{i}\right)\right\}\right]\right) \\
& -\sum_{i=1}^{n} \delta_{i}\left\{\log \sum_{q \in R\left(t_{i}\right)} \exp \left(x_{q}\left[\frac{c_{0}}{2}+\sum_{k=1}^{J}\left\{c_{k} \cos \left(2 k \pi t_{i}\right)+d_{k} \sin \left(2 k \pi t_{i}\right)\right\}\right]\right)\right\} .
\end{aligned}
$$

As estimativas de máxima verossimilhança parcial são encontradas maximizando a função (3.11); no entanto, devido à complexidade da função, procedimentos iterativos de maximização numérica são necessários.

Sejam $\widehat{\theta}$ o vetor que maximiza a função logverossimilhança parcial com respeito a $\theta$ e $\mathbf{U}(\theta) \in \mathbb{R}^{p}$ o vetor escore. O estimador $\widehat{\theta}$ é solução da equação $\mathbf{U}(\theta)=\mathbf{0}$, em que

$$
\mathbf{U}(\theta)=\left(\mathbf{U}_{1}(\theta), \mathbf{U}_{2}^{\mathrm{T}}(\theta)\right)^{\mathrm{T}}
$$

Especificamente,

$$
\mathrm{U}_{1}(\theta)=\frac{\partial \ell(\theta)}{\partial c_{0}} \quad \text { e } \quad \mathbf{U}_{2}(\theta)=\left(\frac{\partial \ell(\theta)}{\partial c_{1}}, \ldots, \frac{\partial \ell(\theta)}{\partial c_{J}}, \frac{\partial \ell(\theta)}{\partial d_{1}}, \ldots, \frac{\partial \ell(\theta)}{\partial d_{J}}\right)^{\mathrm{T}} \in \mathbb{R}^{p-1}
$$

sendo que

$$
\begin{aligned}
& \frac{\partial \ell(\theta)}{\partial c_{0}}=\frac{1}{2} \sum_{i=1}^{n} \delta_{i} x_{i}-\frac{1}{2} \sum_{i=1}^{n} \delta_{i}\left[\frac{\sum_{q \in R\left(t_{i}\right)} x_{q} \exp \left(x_{q} \beta_{0}\left(t_{i}\right)\right)}{\sum_{q \in R\left(t_{i}\right)} \exp \left(x_{q} \beta_{0}\left(t_{i}\right)\right)}\right], \\
& \frac{\partial \ell(\theta)}{\partial c_{k}}=\sum_{i=1}^{n} \delta_{i} x_{i} \cos \left(2 k \pi t_{i}\right)-\sum_{i=1}^{n} \delta_{i}\left[\frac{\sum_{q \in R\left(t_{i}\right)} x_{q} \cos \left(2 k \pi t_{i}\right) \exp \left(x_{q} \beta_{0}\left(t_{i}\right)\right)}{\sum_{q \in R\left(t_{i}\right)} \exp \left(x_{q} \beta_{0}\left(t_{i}\right)\right)}\right]
\end{aligned}
$$

e

$$
\frac{\partial \ell(\theta)}{\partial d_{k}}=\sum_{i=1}^{n} \delta_{i} x_{i} \sin \left(2 k \pi t_{i}\right)-\sum_{i=1}^{n} \delta_{i}\left[\frac{\sum_{q \in R\left(t_{i}\right)} x_{q} \sin \left(2 k \pi t_{i}\right) \exp \left(x_{q} \beta_{0}\left(t_{i}\right)\right)}{\sum_{q \in R\left(t_{i}\right)} \exp \left(x_{q} \beta_{0}\left(t_{i}\right)\right)}\right],
$$

para $k=1, \ldots, J$.

Os métodos para a construção de intervalos de confiança e testes de hipóteses para $\beta_{0}(t)$ são realizados supondo válidos os resultados assintóticos como em Andersen e Gill (1982). 
Para um dado $t \in[0,1)$, propomos estimar a variância de $\widehat{\beta}_{0}(\cdot)$ por

$$
\begin{aligned}
\widehat{\operatorname{var}}\left\{\widehat{\beta}_{0}(t)\right\}= & \frac{1}{4} \widehat{\operatorname{var}}\left(\widehat{c}_{0}\right)+\sum_{k=1}^{J} \cos (2 k \pi t) \widehat{\operatorname{cov}}\left(\widehat{c_{0}}, \widehat{c}_{k}\right)+\sum_{k=1}^{J} \sin (2 k \pi t) \widehat{\operatorname{cov}}\left(\widehat{c}_{0}, \widehat{d}_{k}\right) \\
& +\sum_{j=1}^{J} \sum_{k=1}^{J} \cos (2 j \pi t) \cos (2 k \pi t) \widehat{\operatorname{cov}}\left(\widehat{c}_{j}, \widehat{c}_{k}\right)+\sum_{j=1}^{J} \sum_{k=1}^{J} \sin (2 j \pi t) \sin (2 k \pi t) \widehat{\operatorname{cov}}\left(\widehat{d}_{j}, \widehat{d}_{k}\right) \\
& +\sum_{j=1}^{J} \sum_{k=1}^{J} \cos (2 j \pi t) \sin (2 k \pi t) \widehat{\operatorname{cov}}\left(\widehat{c}_{j}, \widehat{d}_{k}\right) .
\end{aligned}
$$

Supondo a normalidade assintótica do estimador $\widehat{\beta}_{0}(t)$, obtemos o intervalo de confiança para $\beta_{0}(t)$, que pode ser utilizado para testar o efeito de um preditor em um tempo específico $t=t_{0}$. Se um intervalo de $(1-\alpha) \times 100 \%$ de confiança em $t_{0}$ não contém 0 , então a hipótese pontual de não associação $H_{0}: \beta_{0}\left(t_{0}\right)=0$ pode ser rejeitada para um teste bilateral com um nível $\alpha$ de significância. As estimativas de $\widehat{\operatorname{var}}\left(\widehat{c}_{0}\right)$ e das covariâncias na expressão acima são obtidas a partir da inversa da matriz de informação observada.

É importante ressaltar que o impacto de um preditor no risco pode ser nulo, constante ou variante ao longo do tempo. Isso ocorre pois a função (3.9) pode ser tanto nula $\left[\beta_{0}(t)=0\right]$ como constante $\left[\beta_{0}(t)=c_{0} / 2, c_{0} \neq 0\right.$, para todo $\left.t \in[0,1)\right]$. Assim, testes de hipóteses podem ser utilizados para discriminar entre diferentes modelos.

\subsubsection{Teste de hipóteses: riscos proporcionais}

Verificar a adequação do pressuposto de proporcionalidade para uma dada covariável é equivalente a testar se o seu correspondente coeficiente dependente do tempo, $\beta_{0}(t)$, é constante ao longo do tempo. Em vista disso, considere o vetor de parâmetros $\theta=\left(\theta_{1}, \theta_{2}^{\mathrm{T}}\right)^{\mathrm{T}}$, em que $\theta_{1}=c_{0}$ e $\theta_{2}=\left(c_{1}, \ldots, c_{J}, d_{1}, \ldots, d_{J}\right)^{\mathrm{T}} \in$ $\mathbb{R}^{p-1}$. Desse modo, a hipótese nula de interesse é $H_{0}: \theta_{2}=\mathbf{0}$.

Seja $\widehat{\theta}=\left(\widehat{\theta}_{1}, \widehat{\theta}_{2}^{\mathrm{T}}\right)^{\mathrm{T}}$ o estimador de máxima verossimilhança parcial de $\theta$. As três estatísticas frequentemente utilizadas para testar a hipótese $H_{0}$ são

A estatística de teste de Wald é dada por

$$
X_{W}^{2}=\widehat{\theta}_{2}^{\mathrm{T}}\left(\mathbf{I}^{22}(\widehat{\boldsymbol{\theta}})\right)^{-1} \widehat{\boldsymbol{\theta}}_{2},
$$

em que $\mathbf{I}^{22}(\widehat{\boldsymbol{\theta}})$ é a submatriz inferior $(p-1) \times(p-1)$ da inversa da matriz de informação observada $\left[\mathbf{I}^{-1}(\widehat{\boldsymbol{\theta}})\right]$ de dimensão $p \times p$. As expressões dos elementos de $\mathbf{I}(\theta)$ são apresentadas no Apêndice B.

A estatística de teste da razão de verossimilhanças é

$$
X_{R V}^{2}=2\left[\ell(\widehat{\theta})-\ell\left(\widehat{\theta}_{1}(0), \mathbf{0}\right)\right],
$$

em que $\widehat{\theta}_{1}(0)$ é o estimador de máxima verossimilhança parcial de $\beta_{1}$ restrito à hipótese nula.

A estatística de teste do escore é dada por

$$
X_{S C}^{2}=\mathbf{U}_{2}\left(\widehat{\theta}_{1}(0), \mathbf{0}\right)^{\mathrm{T}} \mathbf{I}^{22}\left(\widehat{\boldsymbol{\theta}}_{1}(0), \mathbf{0}\right) \mathbf{U}_{2}\left(\widehat{\boldsymbol{\theta}}_{1}(0), \mathbf{0}\right),
$$

em que $\mathbf{U}_{2}\left(\widehat{\theta}_{1}(0), \mathbf{0}\right) \in \mathbb{R}^{p-1}$ é o vetor escore de $\theta_{2}$ e $\mathbf{I}^{22}\left(\widehat{\theta}_{1}(0), \mathbf{0}\right)$ é a submatriz inferior da inversa da matriz de informação observada avaliados no valor da hipótese nula e restritos no estimador de máxima verossimilhança parcial $\widehat{\theta}_{1}(0)$. 
Para o modelo usual de Cox, de acordo com Klein e Moeschberger (2003), sob algumas condições de regularidade, as três estatísticas acima são assintoticamente equivalentes até primeira ordem, isto é, elas têm, sob a hipótese nula $H_{0}$, a mesma distribuição assintótica $\chi_{(p-1)}^{2}$, em que $(p-1)$ representa os graus de liberdade. Neste trabalho, conjecturamos e avaliamos empiricamente que este resultado também se aplica ao nosso modelo, como pode ser visto no Capítulo 5. Portanto, a hipótese nula é rejeitada para valores grandes de $X_{W}^{2}, X_{R V}^{2}, X_{S C}^{2}$ comparados com o valor crítico $\chi_{(p-1)}^{2}(\alpha)$ obtido da distribuição $\chi_{(p-1)}^{2}$, para um nível de significância nominal $\alpha$ fixado. Detalhes a respeito dessas estatísticas podem ser encontradas em Klein e Moeschberger (2003) e Sen et al. (2010). Em todos os testes adotamos o nível de significância de $5 \%$.

Muitas abordagens têm sido defendidas para avaliar a relevância do pressuposto de proporcionalidade. Dentre os trabalhos que utilizam testes de hipóteses, podemos mencionar Sleeper e Harrington (1990), Gray (1992), Gray (1994), Lin et al. (2006), Huang e Liu (2006) e Saegusa et al. (2014), que tratam o problema via uma representação em bases de splines.

\subsubsection{Predição da função de sobrevivência}

Comumente, o principal objetivo quando se utiliza o modelo de riscos proporcionais de Cox está na estimação do coeficiente da regressão. No entanto, funções relacionadas com o risco basal, $h_{0}(t)$, tais como a função de risco basal acumulada, $H_{0}(t)$, e função de sobrevivência basal, $S_{0}(t)$, também são importantes.

Se a função $h_{0}(t)$ fosse especificada parametricamente, seria possível estimá-la por meio da função de verossimilhança. Porém, na construção da função de verossimilhança parcial, $h_{0}(t)$ é eliminada completamente e, desse modo, os estimadores propostos serão de natureza semiparamétrica.

$\mathrm{Na}$ literatura há algumas propostas de estimador para a função de risco basal após o ajuste do modelo de Cox. Breslow (1972), Cox (1972) e Kalbfleisch e Prentice (1973) propuseram diferentes abordagens para obter $h_{0}(t)$. Cada procedimento dá origem a diferentes estimativas para o risco basal. Por exemplo, Cox (1972) utilizou argumentos da função de verossimilhança condicional para estimar $\beta_{0}$ e propôs uma estimativa pontual para $h_{0}(t)$ que assume valor 0, exceto nos tempos em que ocorrem falhas. Kalbfleisch e Prentice (1973) usaram argumentos da função de verossimilhança marginal para estimar $\beta_{0}$, e consequentemente, propuseram como estimador para o risco basal uma função constante entre convenientes (mas arbitrárias) subdivisões da escala de tempo. Breslow (1972) utilizou raciocínio análogo, porém os eixos dos tempos são divididos em intervalos definidos segundo os tempos de falha.

Neste trabalho, temos também o objetivo de estimar o risco basal, assim como a função de risco basal acumulada para o modelo tipo Cox em que o coeficiente da regressão varia ao longo do tempo (3.10). Para tanto, um estimador da função $H_{0}(t)$, referenciado aqui como estimador de Breslow generalizado, é uma função escada, com saltos nos tempos distintos de falha $\left(t_{1}<\cdots<t_{D}\right)$ e é expresso por

$$
\widehat{H}_{0}(t)=\sum_{l::_{l} \leq t} \widehat{h}_{0}\left(t_{l}\right)
$$

com

$$
\widehat{h}_{0}\left(t_{l}\right)=\frac{1}{\sum_{q \in R\left(t_{l}\right)} \exp \left(x_{q} \widehat{\beta}_{0}\left(t_{l}\right)\right)}, \quad l=1, \ldots, D,
$$

em que $\widehat{\beta}_{0}(\cdot)$ é o estimador de máxima verossimilhança parcial do coeficiente variante ao longo do tempo e $R\left(t_{l}\right)$ representa os índices de todos os indivíduos da amostra que estão em risco no instante de falha $t_{l}^{-}$. 
O estimador de Breslow generalizado (3.12) é uma versão modificada do estimador de Breslow tradicional (Klein e Moeschberger, 2003) em que o estimador invariante ao longo do tempo, $\widehat{\beta}_{0}$, é substituído pelo estimador dependente do tempo, $\widehat{\beta}_{0}(\cdot)$.

Tian et al. (2005) mostraram que (3.12) converge para um processo gaussiano de média nula, em um cenário em que o efeito da covariável sobre a taxa de falha, $\beta_{0}(\cdot)$, é estimada utilizando a função de verossimilhança parcial ponderada por uma função núcleo.

Uma vez obtida a função de risco basal acumulada, é possível obter facilmente o estimador da função de sobrevivência basal, $S_{0}(t)$,

$$
\widehat{S}_{0}(t)=\exp \left[-\sum_{l: t_{l} \leq t} \frac{1}{\sum_{q \in R\left(t_{l}\right)} \exp \left(x_{q} \widehat{\beta}_{0}\left(t_{l}\right)\right)}\right] .
$$

Além de obter as funções mencionadas anteriormente, temos ainda interesse na predição da função de sobrevivência para um valor específico de covariável $x$. Em vista disso, propomos estimar o risco condicional, $h(t \mid x)$, nos $D$ tempos distintos de falha, de modo que o estimador da função de risco condicional é dado por

$$
\widehat{h}\left(t_{l} \mid x\right)=\widehat{h}_{0}\left(t_{l}\right) \exp \left(x \widehat{\beta}_{0}\left(t_{l}\right)\right), \quad l=1, \ldots, D,
$$

em que $\widehat{h}_{0}(\cdot)$ é o estimador da função de risco basal obtido pelo estimador de Breslow generalizado (3.13).

Combinando esses estimadores, o estimador do risco condicional acumulado é

$$
\widehat{H}(t \mid x)=\sum_{l: t_{l} \leq t} \widehat{h}\left(t_{l} \mid x\right)
$$

e, consequentemente, o estimador da função de sobrevivência condicional para um indivíduo específico é dado por

$$
\widehat{S}(t \mid x)=\exp \left(-\sum_{l: t_{l} \leq t} \widehat{h}_{0}\left(t_{l}\right) \exp \left(x \widehat{\beta}_{0}\left(t_{l}\right)\right)\right)
$$

Vale observar que $\widehat{S}_{0}(t)$ assim como $\widehat{S}(t \mid x)$ são funções escada e com saltos nos tempos distintos de falha.

\subsection{Estimação da função de sobrevivência via séries de Fourier}

Na seção anterior, em um cenário semiparamétrico, discutimos a respeito do modelo tipo Cox em que o coeficiente dependente do tempo é representado via bases de Fourier. Nesta seção, adotamos um cenário totalmente paramétrico, em que o risco basal também é expandido em bases de Fourier e, assim, os estimadores propostos serão de natureza paramétrica.

Para isso, vamos também supor que o risco basal é uma função periódica, de período fundamental $L=1$, de tal modo que podemos representar na forma

$$
h_{0}(t)=\frac{a_{0}}{2}+\sum_{k=1}^{J}\left\{a_{k} \cos (2 k \pi t)+b_{k} \sin (2 k \pi t)\right\}, \quad \text { para inteiro } \quad J \geq 1,
$$


em que $a_{0}, a_{k}$ e $b_{k},(k=1, \ldots, J)$, são os coeficientes a serem estimados.

A representação em bases de Fourier permite o risco basal ser constante no intervalo [0,1), isso ocorre se todos os coeficientes $a_{k}^{\prime} s$ e $b_{k}^{\prime} s$ são iguais a 0 . Neste sentido, em situações práticas pode ser interessante verificar se o risco basal é constante ao longo do tempo.

Neste cenário em que as séries de Fourier são utilizadas para representar $h_{0}(t)$ e $\beta_{0}(t)$, a função de risco condicional do modelo de regressão tipo Cox é

$$
h(t \mid x)=\left[\frac{a_{0}}{2}+\sum_{k=1}^{J}\left\{a_{k} \cos (2 k \pi t)+b_{k} \sin (2 k \pi t)\right\}\right] \exp \left(x_{i}\left[\frac{c_{0}}{2}+\sum_{k=1}^{J}\left\{c_{k} \cos (2 k \pi t)+d_{k} \sin (2 k \pi t)\right\}\right]\right),
$$

em que $a_{0}, a_{k}, b_{k}, c_{0}, c_{k}, d_{k},(k=1, \ldots, J)$, são os coeficientes de Fourier a serem estimados.

Observe que expandir as funções $h_{0}(t)$ e $\beta_{0}(t)$ em bases de Fourier pode ser uma vantagem, no sentido de que podemos obter estimativas de $h_{0}(t)$ e $h(t \mid x)$ em qualquer instante de tempo $t \in[0,1)$, ao contrário do que ocorre no cenário semiparamétrico, em que a estimação está restrita apenas aos $D$ tempos distintos de falha.

\subsubsection{Estimação dos parâmetros}

Consideramos a situação em que os tempos de vida não são completamente observados e estão sujeitos a censura à direita. Utilizamos o método de máxima verossimilhança, baseado na função verossimilhança (3.6) para estimar o vetor de parâmetros $\theta=\left(a_{0}, c_{0}, a_{1}, \ldots, a_{J}, b_{1}, \ldots, b_{J}, c_{1}, \ldots, c_{J}, d_{1}, \ldots, d_{J}\right)^{\mathrm{T}} \in \mathbb{R}^{p}$ de dimensão $p=4 J+2$. Para o modelo (3.16), a função logverossimilhança de $\theta$ para estimar $h_{0}(t)$ e $\beta_{0}(t)$ é dada por

$$
\begin{aligned}
\ell(\theta)= & -\sum_{i=1}^{n} \int_{0}^{t_{i}}\left[\frac{a_{0}}{2}+\sum_{k=1}^{J}\left\{a_{k} \cos (2 k \pi s)+b_{k} \sin (2 k \pi s)\right\}\right] \exp \left(x_{i}\left[\frac{c_{0}}{2}+\sum_{k=1}^{J}\left\{c_{k} \cos (2 k \pi s)+d_{k} \sin (2 k \pi s)\right\}\right]\right) d s \\
& +\sum_{i=1}^{n} \delta_{i} \log \left[\frac{a_{0}}{2}+\sum_{k=1}^{J}\left\{a_{k} \cos \left(2 k \pi t_{i}\right)+b_{k} \sin \left(2 k \pi t_{i}\right)\right\}\right]+\sum_{i=1}^{n} \delta_{i} x_{i}\left[\frac{c_{0}}{2}+\sum_{k=1}^{J}\left\{c_{k} \cos \left(2 k \pi t_{i}\right)+d_{k} \sin \left(2 k \pi t_{i}\right)\right\}\right] .
\end{aligned}
$$

Vale observar que o primeiro termo do lado direito em (3.17) não possui primitiva na forma analítica, sendo assim necessários métodos numéricos de integração. Neste trabalho, utilizamos o método de Monte Carlo (Gamerman e Lopes, 2006), que utiliza números aleatórios para avaliar a integral definida, no entanto outros métodos, como as fórmulas de Newton-Cotes e quadratura de Gauss-Legendre podem ser utilizados.

As estimativas de máxima verossimilhança são encontradas maximizando a função (3.17). Porém, devido à complexidade da função de verossimilhança, os estimadores não são obtidos de forma analítica, e assim, por meio de métodos iterativos de maximização numérica as estimativas dos parâmetros são obtidas.

Considere $\widehat{\theta}$ o vetor que maximiza a função logverossimilhança com respeito a $\theta$ e $\mathbf{U}(\theta) \in \mathbb{R}^{p}$ o vetor escore. O estimador $\widehat{\theta}$ é raiz da equação escore $\mathbf{U}(\theta)=\mathbf{0}$, em que

$$
\mathbf{U}(\theta)=\left(\mathrm{U}_{1}(\theta), \mathrm{U}_{2}(\theta), \mathbf{U}_{3}^{\mathrm{T}}(\theta), \mathbf{U}_{4}^{\mathrm{T}}(\theta)\right)^{\mathrm{T}} .
$$

Especificamente,

$$
\mathrm{U}_{1}(\theta)=\frac{\partial \ell(\theta)}{\partial a_{0}}, \quad \mathrm{U}_{2}(\theta)=\frac{\partial \ell(\theta)}{\partial c_{0}}, \quad \mathbf{U}_{3}(\theta)=\left(\frac{\partial \ell(\theta)}{\partial a_{1}}, \ldots, \frac{\partial \ell(\theta)}{\partial a_{J}}, \frac{\partial \ell(\theta)}{\partial b_{1}}, \ldots, \frac{\partial \ell(\theta)}{\partial b_{J}}\right)^{\mathrm{T}} \in \mathbb{R}^{2 J}
$$


e

$$
\mathbf{U}_{4}(\theta)=\left(\frac{\partial \ell(\theta)}{\partial c_{1}}, \ldots, \frac{\partial \ell(\theta)}{\partial c_{J}}, \frac{\partial \ell(\theta)}{\partial d_{1}}, \ldots, \frac{\partial \ell(\theta)}{\partial d_{J}}\right)^{\mathrm{T}} \in \mathbb{R}^{2 J}
$$

sendo que

$$
\begin{aligned}
\frac{\partial \ell(\theta)}{\partial a_{0}} & =\frac{1}{2} \sum_{i=1}^{n} \frac{\delta_{i}}{h_{0}\left(t_{i}\right)}-\frac{1}{2} \sum_{i=1}^{n} \int_{0}^{t_{i}} \exp \left(x_{i} \beta_{0}(s)\right) d s \\
\frac{\partial \ell(\theta)}{\partial c_{0}} & =\frac{1}{2} \sum_{i=1}^{n} \delta_{i} x_{i}-\frac{\partial}{\partial c_{0}} \sum_{i=1}^{n} \int_{0}^{t_{i}} h_{0}(s) \exp \left(x_{i} \beta_{0}(s)\right) d s \\
\frac{\partial \ell(\theta)}{\partial a_{k}} & =\sum_{i=1}^{n} \frac{\delta_{i} \cos \left(2 k \pi t_{i}\right)}{h_{0}\left(t_{i}\right)}-\frac{\partial}{\partial a_{k}} \sum_{i=1}^{n} \int_{0}^{t_{i}} h_{0}(s) \exp \left(x_{i} \beta_{0}(s)\right) d s \\
\frac{\partial \ell(\theta)}{\partial b_{k}} & =\sum_{i=1}^{n} \frac{\delta_{i} \sin \left(2 k \pi t_{i}\right)}{h_{0}\left(t_{i}\right)}-\frac{\partial}{\partial b_{k}} \sum_{i=1}^{n} \int_{0}^{t_{i}} h_{0}(s) \exp \left(x_{i} \beta_{0}(s)\right) d s \\
\frac{\partial \ell(\theta)}{\partial c_{k}} & =\sum_{i=1}^{n} \delta_{i} x_{i} \cos \left(2 k \pi t_{i}\right)-\frac{\partial}{\partial c_{k}} \sum_{i=1}^{n} \int_{0}^{t_{i}} h_{0}(s) \exp \left(x_{i} \beta_{0}(s)\right) d s
\end{aligned}
$$

$\mathrm{e}$

$$
\frac{\partial \ell(\theta)}{\partial d_{k}}=\sum_{i=1}^{n} \delta_{i} x_{i} \sin \left(2 k \pi t_{i}\right)-\frac{\partial}{\partial d_{k}} \sum_{i=1}^{n} \int_{0}^{t_{i}} h_{0}(s) \exp \left(x_{i} \beta_{0}(s)\right) d s
$$

para $k=1, \ldots, J$.

Ao longo do desenvolvimento deste trabalho, deparamo-nos com alguns problemas de ajuste do modelo (3.16). Ocorreram problemas de otimização da função de logverossimilhança (3.17), tanto para dados simulados como para dados reais, encontramos problemas relacionados com a singularidade da matriz hessiana, casos de não convergência dos métodos de otimização, bem como sensibilidade na escolha de valores iniciais para o processo de otimização numérica. Problemas como esses ocorrem também em situações em que a função de risco basal encontra-se próxima de 0 , ocasionando, assim, dificuldades na maximização da função (3.17), uma vez que o termo $h_{0}(t)$ influencia na verossimilhança por meio da função logarítmica.

Em situações em que os problemas mencionados ocorrem, propomos como alternativa estimar os parâmetros do modelo (3.16) em duas etapas.

Etapa 1: Inicialmente, obtemos a estimativa de $\widehat{\beta}_{0}(t)$ utilizando o método de máxima verossimilhança parcial. Nesta etapa, a função de risco basal não é estimada, uma vez que a construção da função de verossimilhança parcial (3.5) elimina totalmente essa componente.

Etapa 2: Obtido o efeito da covariável sobre a taxa de falha, a estimação da função $h_{0}(t)$ é realizada maximizando a função logverossimilhança (3.17). Nesta etapa, o risco basal é obtido via uma pseudoverossimilhança, que é uma função da verossimilhança envolvendo apenas os parâmetros de interesse, a qual é obtida substituindo o vetor de parâmetros de "perturbação" por sua estimativa encontrada na Etapa 1.

Para realizar as duas etapas, seja $\theta=\left(\theta_{1}^{\mathrm{T}}, \theta_{2}^{\mathrm{T}}\right)^{\mathrm{T}}$, em que $\theta_{1}=\left(a_{0}, a_{1}, \ldots, a_{J}, b_{1}, \ldots, b_{J}\right)^{\mathrm{T}} \in \mathbb{R}^{2 J+1}$ e $\theta_{2}=\left(c_{0}, c_{1}, \ldots, c_{J}, d_{1}, \ldots, d_{J}\right)^{\mathrm{T}} \in \mathbb{R}^{2 J+1}$ são os vetores de parâmetros da função de risco basal e do coeficiente dependente do tempo, respectivamente. Na Etapa 1, apenas $\theta_{2}$ é estimado via maximização da função logverossimilhança parcial (3.11), como descrito na Seção 3.3.

Uma vez conhecido $\widehat{\theta}_{2}$, a estimação do risco basal é realizada pelo método de máxima verossimilhança. Porém, nesta etapa a estimação da função $h_{0}(\cdot)$ é baseada na função de verossimilhança envolvendo apenas 
os parâmetros de interesse (pseudo-verossimilhança), a qual é obtida substituindo o vetor de perturbação $\beta_{2}$ pela estimativa de máxima verossimilhança parcial $\widehat{\beta}_{2}$. O logarítmo da pseudo-verossimilhança é dado por

$$
\begin{aligned}
\ell\left(\theta_{1}\right) \propto & -\sum_{i=1}^{n} \int_{0}^{t_{i}}\left[\frac{a_{0}}{2}+\sum_{k=1}^{J}\left\{a_{k} \cos (2 k \pi s)+b_{k} \sin (2 k \pi s)\right\}\right] \exp \left(x_{i}\left[\frac{\widehat{c}_{0}}{2}+\sum_{k=1}^{J}\left\{\widehat{c}_{k} \cos (2 k \pi s)+\widehat{d}_{k} \sin (2 k \pi s)\right\}\right]\right) d s \\
& +\sum_{i=1}^{n} \delta_{i} \log \left[\frac{a_{0}}{2}+\sum_{k=1}^{J}\left\{a_{k} \cos \left(2 k \pi t_{i}\right)+b_{k} \sin \left(2 k \pi t_{i}\right)\right\}\right] .
\end{aligned}
$$

Sejam $\widehat{\theta}_{1}$ o vetor que maximiza a função logverossimilhança com respeito a $\theta_{1}$ e $\mathbf{U}\left(\theta_{1}\right) \in \mathbb{R}^{2 J+1}$ o vetor escore. O estimador $\widehat{\theta}_{1}$ é raiz da equação $\mathbf{U}\left(\theta_{1}\right)=\mathbf{0}$, em que

$$
\mathbf{U}\left(\theta_{1}\right)=\left(\mathrm{U}_{1}\left(\theta_{1}\right), \mathbf{U}_{2}^{\mathrm{T}}\left(\theta_{1}\right)\right)^{\mathrm{T}}
$$

Especificamente,

$$
\mathrm{U}_{1}\left(\beta_{1}\right)=\frac{\partial \ell\left(\theta_{1}\right)}{\partial a_{0}} \quad \text { e } \quad \mathbf{U}_{2}\left(\theta_{1}\right)=\left(\frac{\partial \ell\left(\beta_{1}\right)}{\partial a_{1}}, \ldots, \frac{\partial \ell\left(\beta_{1}\right)}{\partial a_{J}}, \frac{\partial \ell\left(\beta_{1}\right)}{\partial b_{1}}, \ldots, \frac{\partial \ell\left(\beta_{1}\right)}{\partial b_{J}}\right)^{\mathrm{T}} \in \mathbb{R}^{2 J},
$$

sendo que

$$
\begin{aligned}
& \frac{\partial \ell\left(\theta_{1}\right)}{\partial a_{0}}=\frac{1}{2} \sum_{i=1}^{n} \frac{\delta_{i}}{h_{0}\left(t_{i}\right)}-\frac{1}{2} \sum_{i=1}^{n} \int_{0}^{t_{i}} \exp \left(x_{i} \widehat{\beta}_{0}(s)\right) d s, \\
& \frac{\partial \ell\left(\theta_{1}\right)}{\partial a_{k}}=\sum_{i=1}^{n} \frac{\delta_{i} \cos \left(2 k \pi t_{i}\right)}{h_{0}\left(t_{i}\right)}-\frac{\partial}{\partial a_{k}} \sum_{i=1}^{n} \int_{0}^{t_{i}} h_{0}(s) \exp \left(x_{i} \widehat{\beta}_{0}(s)\right) d s
\end{aligned}
$$

$\mathrm{e}$

$$
\frac{\partial \ell\left(\theta_{1}\right)}{\partial b_{k}}=\sum_{i=1}^{n} \frac{\delta_{i} \sin \left(2 k \pi t_{i}\right)}{h_{0}\left(t_{i}\right)}-\frac{\partial}{\partial b_{k}} \sum_{i=1}^{n} \int_{0}^{t_{i}} h_{0}(s) \exp \left(x_{i} \widehat{\beta}_{0}(s)\right) d s
$$

para $k=1, \ldots, J$.

O método para a construção de intervalos de confiança assim como testes de hipóteses para $h_{0}(t)$ e $\beta_{0}(t)$ são realizados supondo válidos os resultados assintóticos. Assim, para um dado $t \in[0,1)$, propomos estimar a variância de $\widehat{\beta}_{0}(\cdot)$ e $\widehat{h}_{0}(\cdot)$, respectivamente, por

$$
\begin{aligned}
\widehat{\operatorname{var}}\left\{\widehat{\beta}_{0}(t)\right\}= & \frac{1}{4} \widehat{\operatorname{var}}\left(\widehat{c}_{0}\right)+\sum_{k=1}^{J} \cos (2 k \pi t) \widehat{\operatorname{cov}}\left(\widehat{c}_{0}, \widehat{c}_{k}\right)+\sum_{k=1}^{J} \sin (2 k \pi t) \widehat{\operatorname{cov}}\left(\widehat{c}_{0}, \widehat{d}_{k}\right) \\
& +\sum_{j=1}^{J} \sum_{k=1}^{J} \cos (2 j \pi t) \cos (2 k \pi t) \widehat{\operatorname{cov}}\left(\widehat{c}_{j}, \widehat{c}_{k}\right)+\sum_{j=1}^{J} \sum_{k=1}^{J} \sin (2 j \pi t) \sin (2 k \pi t) \widehat{\operatorname{cov}}\left(\widehat{d}_{j}, \widehat{d}_{k}\right) \\
& +\sum_{j=1}^{J} \sum_{k=1}^{J} \cos (2 j \pi t) \sin (2 k \pi t) \widehat{\operatorname{cov}}\left(\widehat{c}_{j}, \widehat{d}_{k}\right)
\end{aligned}
$$


e

$$
\begin{aligned}
\widehat{\operatorname{var}}\left\{\widehat{h}_{0}(t)\right\}= & \frac{1}{4} \widehat{\operatorname{var}}\left(\widehat{a}_{0}\right)+\sum_{k=1}^{J} \cos (2 k \pi t) \widehat{\operatorname{cov}}\left(\widehat{a}_{0}, \widehat{a}_{k}\right)+\sum_{k=1}^{J} \sin (2 k \pi t) \widehat{\operatorname{cov}}\left(\widehat{a}_{0}, \widehat{b}_{k}\right) \\
& +\sum_{j=1}^{J} \sum_{k=1}^{J} \cos (2 j \pi t) \cos (2 k \pi t) \widehat{\operatorname{cov}}\left(\widehat{a}_{j}, \widehat{a}_{k}\right)+\sum_{j=1}^{J} \sum_{k=1}^{J} \sin (2 j \pi t) \sin (2 k \pi t) \widehat{\operatorname{cov}}\left(\widehat{b}_{j}, \widehat{b}_{k}\right) \\
& +\sum_{j=1}^{J} \sum_{k=1}^{J} \cos (2 j \pi t) \sin (2 k \pi t) \widehat{\operatorname{cov}}\left(\widehat{a}_{j}, \widehat{b}_{k}\right) .
\end{aligned}
$$

Assumindo que vale a normalidade assintótica dos estimadores de $\theta$, um intervalo de confiança para $\beta_{0}(t)$ pode ser obtido, que pode ser utilizado para testar o efeito de um preditor em um tempo específico $t=t_{0}$. Além disso, um intervalo de confiança para o risco basal também pode ser construído, para $t$ prefixado.

É importante observar que, em situações práticas em que o risco basal é próximo de 0 , o procedimento de otimização em duas etapas pode ser ineficiente, e uma abordagem em um cenário semiparamétrico pode ser mais conveniente, vide Seção 3.3.

\subsubsection{Testes de hipóteses: riscos proporcionais e/ou risco basal constante}

O modelo proposto (3.16) é bastante flexível no sentido de incorporar riscos não proporcionais para uma dada covariável. No entanto, podemos verificar se a proporcionalidade para uma dada covariável se sustenta, bem como avaliar se a função de risco basal é constante ao longo do tempo.

Para testar essas hipóteses, primeiramente consideramos o caso em que a estimação dos parâmetros é realizada em apenas uma etapa. Se o objetivo inicial é avaliar a suposição de riscos proporcionais, (3.10), considere $\theta=\left(\theta_{1}^{\mathrm{T}}, \theta_{2}^{\mathrm{T}}\right)^{\mathrm{T}}$, em que $\theta_{1}=\left(a_{0}, c_{0}, a_{1}, \ldots, a_{J}, b_{1}, \ldots, b_{J}\right)^{\mathrm{T}} \in \mathbb{R}^{2 J+2}$ e $\theta_{2}=\left(c_{1}, \ldots, c_{J}, d_{1}, \ldots, d_{J}\right)^{\mathrm{T}} \in$ $\mathbb{R}^{2 J}$. Avaliar a proporcionalidade dos riscos para uma dada covariável é equivalente a testar $H_{0}: \theta_{2}=\mathbf{0}$.

Por outro lado, se o propósito é testar a função de risco basal constante ao longo do tempo, (3.15), considere $\theta_{1}=\left(a_{0}, c_{0}, c_{1}, \ldots, c_{J}, d_{1}, \ldots, d_{J}\right)^{\mathrm{T}} \in \mathbb{R}^{2 J+2}$ e $\theta_{2}=\left(a_{1}, \ldots, a_{J}, b_{1}, \ldots, b_{J}\right)^{\mathrm{T}} \in \mathbb{R}^{2 J}$ e, então, a hipótese nula é $H_{0}: \theta_{2}=\mathbf{0}$.

Em circunstâncias em que a estimação dos parâmetros é realizada utilizando o procedimento em duas etapas, a verificação de riscos proporcionais para uma dada covariável, assim como função de risco basal constante ao longo do tempo, segue de forma análoga à Seção 3.3.

Para testar as hipóteses de interesse, utilizamos os três testes (Wald, razão de verossimilhanças ou escore) mencionados na Seção 3.3.2. No entanto, é importante destacar que as estatísticas dos testes são utilizadas heuristicamente pois, neste trabalho temos indicações que elas são assintoticamente equivalentes e que sob, a hipótese nula, possuem a mesma distribuição.

\subsubsection{Predição da função de sobrevivência}

Como a função $h_{0}(t)$ é especificada por uma expansão em séries de Fourier (3.15), os estimadores propostos serão de natureza paramétrica. Utilizando as relações matemáticas entre as funções básicas de sobrevivência, os estimadores da função de risco basal acumulada e sobrevivência basal são, respectivamente,

$$
\widehat{H}_{0}(t)=\int_{0}^{t} \widehat{h}_{0}(s) d s=\frac{\widehat{a}_{0}}{2} t+\frac{1}{2 k \pi} \sum_{k=1}^{J}\left[\widehat{a}_{k} \sin (2 k \pi t)+\widehat{b}_{k}\{1-\cos (2 k \pi t)\}\right]
$$


e

$$
\widehat{S}_{0}(t)=\exp \left(-\frac{\widehat{a}_{0}}{2} t-\frac{1}{2 k \pi} \sum_{k=1}^{J}\left[\widehat{a}_{k} \sin (2 k \pi t)+\widehat{b}_{k}\{1-\cos (2 k \pi t)\}\right]\right),
$$

em que $\widehat{a}_{0}, \widehat{a}_{k} \mathrm{e} \widehat{b}_{k},(k=1, \ldots, J)$, são os estimadores de máxima verossimilhança.

Além das funções usuais da análise de sobrevivência, a predição da função de sobrevivência para um valor específico da covariável $x$ também é de grande interesse, como já discutido anteriormente.

É importante mencionar que a representação em séries de Fourier para $h_{0}(\cdot)$ e $\beta_{0}(\cdot)$ impossibilita obter o risco condicional acumulado de maneira tradicional (tempos contínuos), pois não há primitiva na forma explícita para a função $H(t \mid x)=\int_{0}^{t} h_{0}(s) \exp \left(x \beta_{0}(s)\right) d s$; desse modo, discretizamos o intervalo de tempo nos $D$ tempos distintos de falha, de tal modo que o risco condicional é obtido por

$$
\widehat{h}\left(t_{l} \mid x\right)=\widehat{h}_{0}\left(t_{l}\right) \exp \left(x \widehat{\beta}_{0}\left(t_{l}\right)\right), \quad l=1, \ldots, D,
$$

em que $\widehat{h}_{0}(\cdot)$ e $\widehat{\beta}_{0}(\cdot)$ são os estimadores de máxima verossimilhança da função de risco basal e do coeficiente dependente do tempo, respectivamente.

O estimador do risco condicional acumulado é facilmente obtido e é dado por

$$
\widehat{H}(t \mid x)=\sum_{l: t_{l} \leq t} \widehat{h}_{0}\left(t_{l}\right) \exp \left(x \widehat{\beta}_{0}\left(t_{l}\right)\right)
$$

e, consequentemente, para um valor específico da covariável $x$, o estimador da função de sobrevivência condicional é

$$
\widehat{S}(t \mid x)=\exp \left[-\sum_{l: t_{l} \leq t} \widehat{h}_{0}\left(t_{l}\right) \exp \left(x \widehat{\beta}_{0}\left(t_{l}\right)\right)\right]
$$

Podemos observar que representar o risco basal em bases de Fourier implica em estimadores com expressões analíticas explícitas (dependendo apenas dos parâmetros), ao contrário do cenário semiparamétrico, em que os estimadores dependem também do estimador de Breslow generalizado, exceto o coeficiente variante ao longo do tempo. Além disso, vale destacar que a estimação da função de risco condicional (3.18) não está restrita apenas aos tempos de falha, como ocorre no cenário semiparamétrico (3.14), sendo assim uma vantagem abordar o problema em um cenário paramétrico. 


\section{Capítulo 4}

\section{Modelo de regressão tipo Cox via ondaletas de Haar}

Neste capítulo, abordamos inicialmente em um cenário semiparamétrico, a estimação do efeito temporal da covariável sobre a taxa de falha por meio das ondaletas de Haar e, em seguida, a função de risco basal é também especificada via uma representação em séries de ondaletas de Haar.

Diferentemente do que acontece na maioria dos trabalhos que utilizam ondaletas, preferimos adotar apenas a base de ondaletas de Haar, porque além de manter certa similaridade com as séries de Fourier, como ortogonalidade entre as funções, expressões analíticas das bases e ainda permitir avaliar a proporcionalidade dos riscos via testes de hipóteses, beneficiamo-nos do fato de as ondaletas serem localizadas em tempo e frequência, simultaneamente, ao contrário das séries de Fourier, que são localizadas apenas em frequência.

O presente capítulo está organizado como segue. Na Seção 4.1, apresentamos o modelo de regressão tipo Cox em que o coeficiente dependente do tempo é expandido via séries de ondaletas de Haar. Discutimos a respeito da estimação dos parâmetros pelo método de máxima verossimilhança parcial. Além disso, propomos um procedimento de avaliação do pressuposto de proporcionalidade dos riscos para uma dada covariável, por meio de testes de hipóteses. Posteriormente, descrevemos sobre a predição da função de sobrevivência para um indivíduo específico. Na Seção 4.2, consideramos uma representação em bases de ondaletas para a função de risco basal, assim como para o efeito dependente do tempo. Utilizamos o método de máxima verossimilhança para estimar os parâmetros do modelo. Discutimos, também, via testes de hipóteses, um procedimento para avaliar a proporcionalidade dos riscos e também o risco basal constante ao longo do tempo. Finalizando o capítulo, apresentamos um estimador da função de sobrevivência condicional baseado nas ondaletas.

\subsection{Coeficiente dependente do tempo via ondaletas de Haar}

Nesta seção, propomos um modelo tipo Cox em que o efeito da covariável sobre a taxa de falha é modelado via ondaletas de Haar e a função de risco basal não especificada, apesar de não negativa. Ao longo do texto, consideramos apenas as ondaletas clássicas, no entanto, outras classes de ondaletas, como as deformadas podem ser adotadas (vide Capítulo 2).

Supomos que o coeficiente de regressão do modelo (3.4) é uma função quadrado integrável no intervalo $[0,1]$, isto é, $\beta_{0} \in L_{2}([0,1])$, de modo que $\beta_{0}(\cdot)$ possa ser representada em bases de ondaletas de Haar, como 
em (2.3). A função de risco condicional do modelo é dada por

$$
h(t \mid x)=h_{0}(t) \exp \left(x\left\{c \phi_{0,0}(t)+\sum_{j=0}^{J} \sum_{k=0}^{2^{j}-1} d_{j, k} \psi_{j, k}(t)\right\}\right),
$$

em que $h_{0}(\cdot)$ é a função de risco basal completamente não especificada, $\phi_{0,0}(\cdot)$ e $\left\{\psi_{j, k}(\cdot), j=0, \ldots, J ; k=\right.$ $\left.0, \ldots, 2^{j}-1\right\}$ são funções conhecidas representando as ondaletas pai e mãe, respectivamente, enquanto $c, d_{0,0}, \ldots, d_{J, 2^{J}-1}$, são parâmetros a serem estimados.

É importante observar que, além da tradicional base de ondaletas de Haar, na literatura há diversas outras bases, como as daublets, symmlets e coiflets. No entanto, adotar qualquer outra base inviabiliza, por meio de testes de hipóteses, avaliar se a proporcionalidade dos riscos para uma dada covariável se sustenta, como mencionado no Capítulo 2.

Como destacamos, as ondaletas $\phi_{0,0}(\cdot)$ e $\psi_{j, k}(\cdot)$ assumem valores não nulos apenas no intervalo $[0,1)$, sendo assim necessário o redimensionamento dos tempos observados para esse intervalo. Neste trabalho, a transformação da escala de tempo é realizada por meio de dois procedimentos, que denominaremos por clássico e deformado. Antes de descrevê-los, considere $t_{(1)}<\cdots<t_{(n)}$ os tempos observados (falha ou censura) ordenados e sejam $t_{(1)}^{*}<\cdots<t_{(n)}^{*}$ os tempos transformados e ordenados. Os dois procedimentos utilizados são descritos como segue:

1. Clássico: Os tempos transformados são obtidos dividindo todos os tempos observados pelo maior tempo observado, como segue

$$
t_{(k)}^{*}=\frac{t_{(k)}}{t_{(n)}}, \quad k=1, \ldots, n
$$

2. Deformado: Os tempos reescalonados são obtidos utilizando a função de distribuição empírica, $F$, em que

$$
t_{(k)}^{*}=\widehat{F}\left(t_{(k)}\right)=\frac{1}{n} \sum_{i=1}^{n} \mathbb{I}_{\left\{t_{(i)} \leq t_{(k)}\right\}}=\frac{k}{n}, \quad k=1, \ldots, n
$$

As técnicas expostas anteriormente apresentam diferenças no modo em que os tempos são redimensionados para o intervalo desejado. O procedimento clássico apenas reescalona os tempos observados, enquanto a técnica deformada trata os tempos transformados em instantes igualmente espaçados.

Observe ainda que esses métodos transformam o maior tempo observado $t_{(n)}$ em $t_{(n)}^{*}=1$, no entanto como as ondaletas pai e mãe são nulas em 1 , adotamos para $t_{(n)}^{*}$ um valor imediatamente anterior a 1 , de modo que todos os tempos reescalonados estejam no intervalo $[0,1)$.

Em consequência da definição da função de Haar (2.2), o coeficiente da regressão é assumido ser constante entre subdivisões da escala de tempo (igualmente espaçados). É importante destacar que o número de subintervalos e de parâmetros estão relacionados com o nível de resolução $J$ desejado. Para um dado nível fixado, $J=J_{0} \geq 0$, a quantidade de subdivisões do intervalo $[0,1)$ bem como o número de parâmetros do modelo é $p=2^{J_{0}+1}$. Logo, para o acréscimo de uma unidade em $J$ o número de subintervalos e parâmetros duplicam, e assim, menores amplitudes e saltos e, consequentemente maior detalhe de $\beta_{0}(t)$ serão observados, porém um número maior de parâmetros deverá ser estimado.

Vale ainda ressaltar que o modelo (4.1) generaliza o modelo de riscos proporcionais, pois permite riscos não proporcionais, a menos que a função $\beta_{0}(t)$ seja constante ao longo do tempo, ou seja, quando os coeficientes de detalhes $d_{0,0}, \ldots, d_{J, 2^{J}-1}$ são todos iguais a zero. 


\subsubsection{Estimação do efeito da covariável via ondaletas Haar}

Para obter a estimativa do coeficiente dependente do tempo, utilizamos o método de máxima verossimilhança parcial. Neste cenário, o vetor de parâmetros do modelo é composto pelos coeficientes das ondaletas pai e mãe, $\theta=\left(c, d_{0,0}, \ldots, d_{J, 2^{J}-1}\right)^{\mathrm{T}} \in \mathbb{R}^{p}$ de dimensão $p=2^{J+1}$. A função logverossimilhança parcial de $\theta$ para estimar $\beta_{0}(t)$ é dada por

$$
\begin{aligned}
\ell(\theta)= & \sum_{i=1}^{n} \delta_{i}\left[x_{i}\left\{c \phi_{0,0}\left(t_{i}\right)+\sum_{j=0}^{J} \sum_{k=0}^{2^{j}-1} d_{j, k} \psi_{j, k}\left(t_{i}\right)\right\}\right] \\
& -\sum_{i=1}^{n} \delta_{i}\left(\log \sum_{q \in R\left(t_{i}\right)} \exp \left[x_{q}\left\{c \phi_{0,0}\left(t_{i}\right)+\sum_{j=0}^{J} \sum_{k=0}^{2^{j}-1} d_{j, k} \psi_{j, k}\left(t_{i}\right)\right\}\right]\right) .
\end{aligned}
$$

Devido à complexidade da função (4.2), as estimativas dos parâmetros são obtidas por métodos iterativos de maximização numérica. Considere o vetor $\widehat{\theta}$ que maximiza a função logverossimilhança parcial com respeito a $\theta$ e seja $\mathbf{U}(\theta) \in \mathbb{R}^{p}$ o vetor escore. O estimador $\widehat{\theta}$ é solução da equação escore $\mathbf{U}(\theta)=\mathbf{0}$, em que

$$
\mathbf{U}(\theta)=\left(\mathrm{U}_{1}(\theta), \mathbf{U}_{2}^{\mathrm{T}}(\theta)\right)^{\mathrm{T}}
$$

com

$$
\mathrm{U}_{1}(\theta)=\frac{\partial \ell(\theta)}{\partial c} \quad \text { e } \quad \mathbf{U}_{2}(\theta)=\left(\frac{\partial \ell(\theta)}{\partial d_{0,0}}, \ldots, \frac{\partial \ell(\theta)}{\partial d_{J, 2^{J}-1}}\right)^{\mathrm{T}} \in \mathbb{R}^{p-1}
$$

sendo que

$$
\frac{\partial \ell(\theta)}{\partial c}=\sum_{i=1}^{n} \delta_{i} x_{i} \phi_{0,0}\left(t_{i}\right)-\sum_{i=1}^{n} \delta_{i}\left[\frac{\sum_{q \in R\left(t_{i}\right)} x_{q} \phi_{0,0}\left(t_{i}\right) \exp \left(x_{q} \beta_{0}\left(t_{i}\right)\right)}{\sum_{q \in R\left(t_{i}\right)} \exp \left(x_{q} \beta_{0}\left(t_{i}\right)\right)}\right]
$$

e

$$
\frac{\partial \ell(\theta)}{\partial d_{j, k}}=\sum_{i=1}^{n} \delta_{i} x_{i} \psi_{j, k}\left(t_{i}\right)-\sum_{i=1}^{n} \delta_{i}\left[\frac{\sum_{q \in R\left(t_{i}\right)} x_{q} \psi_{j, k}\left(t_{i}\right) \exp \left(x_{q} \beta_{0}\left(t_{i}\right)\right)}{\sum_{q \in R\left(t_{i}\right)} \exp \left(x_{q} \beta_{0}\left(t_{i}\right)\right)}\right],
$$

para $j=0, \ldots, J ; k=0, \ldots, 2^{j}-1$.

A construção de intervalos de confiança, assim como testes de hipóteses para $\beta_{0}(t)$ são realizados supondo válidos os resultados assintóticos, como em Andersen e Gill (1982). Assim, para um dado $t \in[0,1)$, propomos estimar a variância de $\widehat{\beta}_{0}(\cdot)$ por

$$
\begin{aligned}
\widehat{\operatorname{var}}\left\{\widehat{\beta}_{0}(t)\right\}= & \left\{\phi_{0,0}(t)\right\}^{2} \widehat{\operatorname{var}}(\widehat{c})+2 \phi_{0,0}(t) \sum_{j=0}^{J} \sum_{k=0}^{2^{j}-1} \psi_{j, k}(t) \widehat{\operatorname{cov}}\left(\widehat{c}, \widehat{d}_{j, k}\right) \\
& +\widehat{\operatorname{cov}}\left\{\sum_{j=0}^{J} \sum_{k=0}^{2^{j}-1} \widehat{d}_{j, k} \psi_{j, k}(t), \sum_{j=0}^{J} \sum_{k=0}^{2^{j}-1} \widehat{d}_{j, k} \psi_{j, k}(t)\right\} .
\end{aligned}
$$

Supondo a normalidade assintótica dos estimadores de $\widehat{\beta}$, construímos um intervalo de confiança para $\beta_{0}(\cdot)$. Como mencionamos no Capítulo 3, podemos testar o efeito de uma covariável em um tempo específico $t=t_{0}$ utilizando o intervalo de confiança, bem como verificar a proporcionalidade dos riscos para uma dada covariável por meio de testes de hipóteses. As estimativas de $\widehat{\operatorname{var}}(\widehat{c})$ e das covariâncias na expressão 
acima são obtidas a partir da inversa da matriz de informação observada dada no Apêndice B.

\subsubsection{Teste de hipóteses: riscos proporcionais}

Como destacamos, o modelo (4.1) generaliza o modelo de riscos proporcionais de Cox, e assim, verificar se a proporcionalidade dos riscos se sustenta é análogo a testar se, $\beta_{0}(t)=c, c \neq 0$, para todo $t \in[0,1)$. Para isso, considere o vetor de parâmetros $\theta=\left(\theta_{1}, \theta_{2}^{\mathrm{T}}\right)^{\mathrm{T}}$, em que $\theta_{1}=c$ e $\theta_{2}=\left(d_{0,0}, \ldots, d_{J, 2^{J}-1}\right)^{\mathrm{T}} \in \mathbb{R}^{p-1}$. Logo, a hipótese de interesse é $H_{0}: \theta_{2}=\mathbf{0}$. Os testes de Wald, razão de verossimilhanças e escore são utilizados para testar a hipótese, e seguem de forma equivalente como exposto na Seção 3.3.2. As expressões dos elementos da matriz de informação observada, $\mathbf{I}(\theta)$, são apresentadas no Apêndice B.

\subsubsection{Predição da função de sobrevivência}

Além de estimar o efeito da covariável sobre a taxa de falha, temos também o interesse em obter predições da função de sobrevivência para um indivíduo específico. A fim de obter um estimador para a função de sobrevivência condicional, utilizamos raciocínio análogo ao apresentado no Capítulo 3, em que após ajustar o modelo (4.1), a função de risco basal acumulada é obtida via o estimador de Breslow generalizado

$$
\widehat{H}_{0}(t)=\sum_{l::_{l} \leq t} \widehat{h}_{0}\left(t_{l}\right)
$$

com

$$
\widehat{h}_{0}\left(t_{l}\right)=\frac{1}{\sum_{q \in R\left(t_{l}\right)} \exp \left(x_{q} \widehat{\beta}_{0}\left(t_{l}\right)\right)}, \quad l=1, \ldots, D,
$$

em que $t_{1}, \ldots, t_{D}$ são os tempos de falha (distintos e ordenados) e $\widehat{\beta}_{0}(\cdot)$ é o estimador de máxima verossimilhança parcial de $\beta_{0}(\cdot)$.

Uma vez obtida a estimativa da função de risco basal acumulada, a função de sobrevivência basal, $\widehat{S}_{0}(\cdot)$, é facilmente encontrada e é expressa por

$$
\widehat{S}_{0}(t)=\exp \left(-\sum_{l: t_{l} \leq t} \frac{1}{\sum_{q \in R\left(t_{l}\right)} \exp \left(x_{q} \widehat{\beta}_{0}\left(t_{l}\right)\right)}\right) .
$$

Antes de propor um estimador para a função de sobrevivência condicional, apresentamos um estimador para a função de risco condicional e também para o risco condicional acumulado.

Inicialmente, através das relações matemáticas entre as funções básicas da análise de sobrevivência, o estimador da função de risco condicional acumulado é

$$
\widehat{H}(t \mid x)=\int_{0}^{t} \widehat{h}_{0}(s) \exp \left(x \widehat{\beta}_{0}(s)\right) d s .
$$

A integral definida em (4.6) depende da função de risco basal (não especificada parametricamente) e também do coeficiente dependente do tempo, tornando complicado ou até mesmo impossível obter uma expressão simplificada para $\widehat{H}(t \mid x)$. Todavia, como a função $\beta_{0}(\cdot)$ é representada em bases de ondaletas de Haar, o estimador do risco condicional acumulado, $\widehat{H}(t \mid x)$, pode ser obtido facilmente.

A fim de exemplificar o procedimento, fixamos o nível de resolução em $J=0$, de modo que o estimador 
da função $\beta_{0}(\cdot)$ é reescrito por

$$
\widehat{\beta}_{0}(t)=\left\{\begin{array}{lll}
\hat{c}+\hat{d}, & \text { se } & 0 \leq t<0,5 \\
\hat{c}-\hat{d}, & \text { se } & 0,5 \leq t<1
\end{array}\right.
$$

em que $\hat{c}$ e $\hat{d}$ são os estimadores de máxima verossimilhança parcial de $c$ e $d$.

Para um valor específico da covariável $x$, o estimador da função de risco condicional nos tempos distintos de falha $\left(t_{l}, l=1, \ldots, D\right)$ é

$$
\widehat{h}\left(t_{l} \mid x\right)=\left\{\begin{array}{lll}
\widehat{h}_{0}\left(t_{l}\right) \exp \{x(\hat{c}+\hat{d})\}, & \text { se } & 0 \leq t_{l}<0,5 \\
\widehat{h}_{0}\left(t_{l}\right) \exp \{x(\hat{c}-\hat{d})\}, & \text { se } & 0,5 \leq t_{l}<1
\end{array}\right.
$$

em que $\widehat{h}_{0}(\cdot)$ é o estimador da função de risco basal (4.4).

Consequentemente, o estimador da função de risco condicional acumulada (4.6) é expresso por

$$
\widehat{H}(t \mid x)=\left\{\begin{array}{lll}
\widehat{H}_{0}(t) \exp (x(\hat{c}+\hat{d})), & \text { se } & 0 \leq t<0,5, \\
\widehat{H}_{0}(0,5)[\exp (x(\hat{c}+\hat{d}))-\exp (x(\hat{c}-\hat{d}))]+\widehat{H}_{0}(t) \exp (x(\hat{c}-\hat{d})), & \text { se } & 0,5 \leq t<1
\end{array}\right.
$$

em que $\widehat{H}_{0}(\cdot)$ é o estimador de Breslow generalizado (4.3).

Portanto, o estimador da função de sobrevivência para um específico indivíduo é

$$
\widehat{S}(t \mid x)=\left\{\begin{array}{lll}
\left\{\widehat{S}_{0}(t)\right\}^{\exp (x(\hat{c}+\hat{d}))}, & \text { se } & 0 \leq t<0,5, \\
\left\{\widehat{S}_{0}(0,5)\right\}^{\exp (x(\hat{c}+\hat{d}))-\exp (x(\hat{c}-\hat{d}))}\left\{\hat{S}_{0}(t)\right\}^{\exp (x(\hat{c}-\hat{d}))}, & \text { se } & 0,5 \leq t<1,
\end{array}\right.
$$

em que $\widehat{S}_{0}(\cdot)$ é o estimador da função de sobrevivência basal obtido em (4.5).

Como mencionado anteriormente, os estimadores expostos são específicos para o nível de resolução $J=0$; no entanto, tais estimadores podem ser obtidos para qualquer $J \geq 0$. Antes de prosseguir com a generalização, a fim de facilitar a notação, considere o vetor $\widehat{\mathbf{b}}=\left(\widehat{b}_{1}, \ldots, \widehat{b}_{p}\right)^{\mathrm{T}} \in \mathbb{R}^{p}$, tal que

$$
\widehat{b}_{k}=\widehat{\beta}_{0}(t) \mathbb{I}_{\{(k-1) / p \leq t<k / p\}}, \quad k=1, \ldots, p=2^{J+1},
$$

em que II representa à função indicadora no intervalo definido, $\widehat{\beta}_{0}(\cdot)$ é o estimador de máxima verossimilhança parcial e $\widehat{b}_{k}$ representa o estimador do coeficiente dependente do tempo no subintervalo [ $k$ $1) / p, k / p)$. A Figura 4.1 ilustra, para um exemplo fictício, as componentes do vetor $\widehat{\mathbf{b}}$ para o nível de resolução $J=2$.

Utilizando essa notação, o estimador do coeficiente dependente do tempo pode ser reescrito na forma

$$
\widehat{\beta}_{0}(t)=\sum_{k=1}^{p} \widehat{b}_{k} \mathbb{I}_{\{(k-1) / p \leq t<k / p\}}
$$

e, assim, para um valor específico da covariável $x$, o risco condicional nos $D$ tempos de falha distintos é

$$
\widehat{h}\left(t_{l} \mid x\right)=\widehat{h}_{0}\left(t_{l}\right) \sum_{k=1}^{p} \exp \left(x \widehat{b}_{k}\right) \mathbb{I}_{\left\{(k-1) / p \leq t_{l}<k / p\right\}}, \quad l=1, \ldots, D .
$$

Desta maneira, utilizando (4.6) e (4.7) e após algumas manipulações matemáticas, o estimador da 


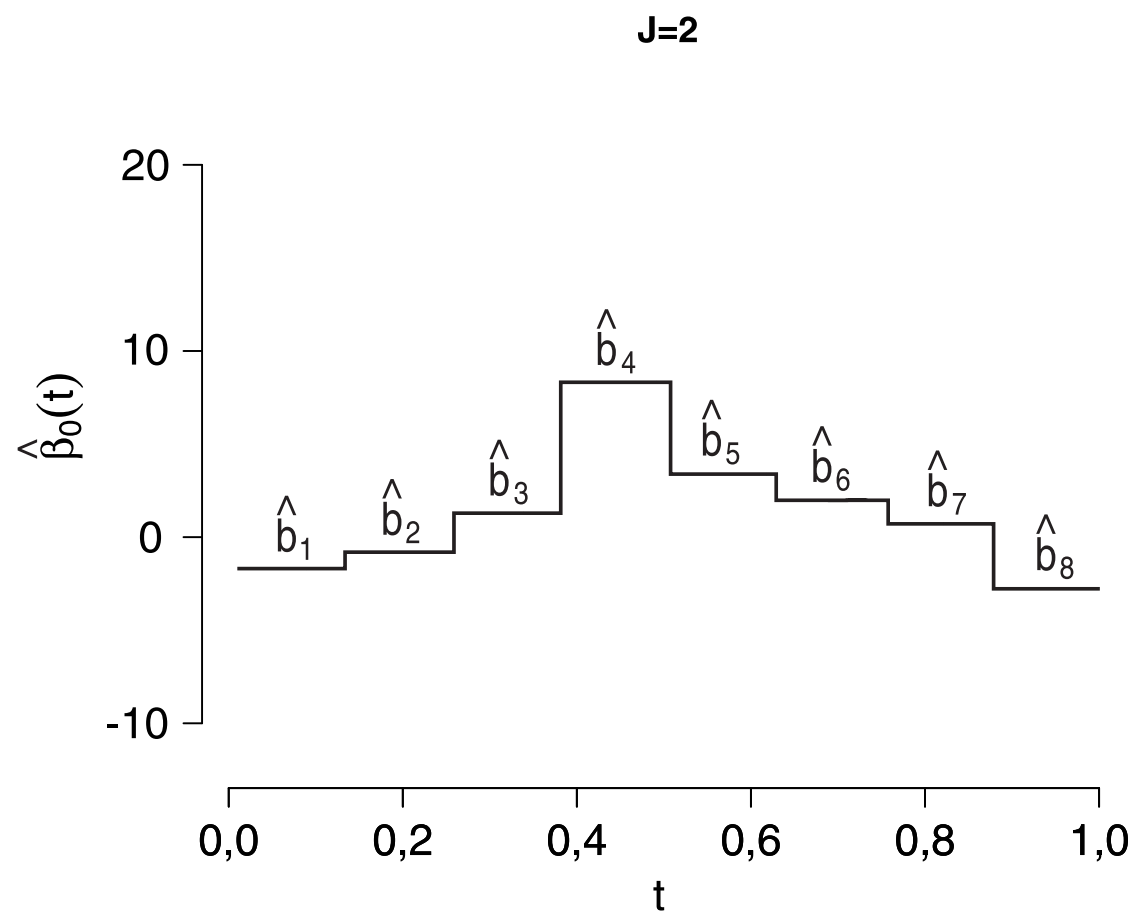

Figura 4.1: Estimativa do efeito da covariável ao longo do tempo (linha sólida) obtido via bases de ondaletas Haar com nível de resolução $J=2$.

função de risco condicional acumulada é dado por

$$
\begin{aligned}
\widehat{H}(t \mid x)= & \sum_{k=2}^{p} \sum_{j=1}^{k-1} \widehat{H}_{0}(j / p)\left\{\exp \left(x \widehat{b}_{j}\right)-\exp \left(x \widehat{b}_{j+1}\right)\right\} \mathbb{I}_{\{(k-1) / p \leq t<k / p\}} \\
& +\widehat{H}_{0}(t) \sum_{k=1}^{p} \exp \left(x \widehat{b}_{k}\right) \mathbb{I}_{\{(k-1) / p \leq t<k / p\}}
\end{aligned}
$$

Portanto, o estimador da função de sobrevivência condicional para um indivíduo específico é

$$
\begin{aligned}
\widehat{S}(t \mid x)= & \exp \left[\sum_{k=2}^{p} \sum_{j=1}^{k-1} \widehat{H}_{0}(j / p)\left\{\exp \left(x \widehat{b}_{j+1}\right)-\exp \left(x \widehat{b}_{j}\right)\right\} \mathbb{I}_{\{(k-1) / p \leq t<k / p\}}\right] \\
& \times\left\{\widehat{S}_{0}(t)\right\}^{\sum_{k=1}^{p} \exp \left(\widehat{x b}_{k}\right) \mathbb{I}_{\{(k-1) / p \leq t<k / p\}}} .
\end{aligned}
$$

É importante observar que, devido à complexidade da expressão do estimador (4.8), não obtemos uma expressão explícita para a variância de $\widehat{S}(t \mid x)$, para um dado $t \in[0,1)$. No entanto, uma alternativa para se obter o intervalo de confiança de $\widehat{S}(t \mid x)$ é utilizar as técnicas de reamostragem, como bootstrap e jackknife. Nesta tese, o intervalo de confiança pontual de 95\% para $\widehat{S}(t \mid x)$, para $t$ prefixado, é obtido por meio dos percentis da reamostragem bootstrap, em que é baseado em 1000 reamostras com reposição (Davison e Hinkley, 1997; Efron e Tibshirani, 1993). 


\subsection{Estimação da função de sobrevivência via ondaletas de Haar}

Na seção anterior, em um cenário semiparamétrico, abordamos a estimação do efeito da covariável sobre a taxa de falha por meio das ondaletas. Nesta seção, consideramos o problema em um cenário paramétrico, em que a função de risco basal também é expandida em bases de ondaletas Haar e, assim, os estimadores propostos serão de natureza paramétrica.

Para tanto, vamos também supor que a função de risco basal do modelo (4.1) é uma função quadrado integrável em $[0,1]$, de tal modo que $h_{0}(\cdot)$ é representada via séries de ondaletas de Haar

$$
h_{0}(t)=a \phi_{0,0}(t)+\sum_{j=0}^{J} \sum_{k=0}^{2^{j}-1} b_{j, k} \psi_{j, k}(t)
$$

em que $J$ representa o nível de resolução, enquanto $a, b_{0,0}, \ldots, b_{J, 2^{J}-1}$ são os coeficientes a serem estimados.

Devido à definição da função de Haar (2.2), o risco basal é assumido constante entre subdivisões da escala de tempo. Além disso, a representação em bases de ondaletas permite a função de risco basal ser constante ao longo do tempo, caso os coeficientes $b_{j, k}^{\prime} s$ sejam todos nulos.

Considerando o cenário em que as bases de ondaletas de Haar são utilizadas para representar $h_{0}(t) \mathrm{e}$ $\beta_{0}(t)$, a função de risco condicional do modelo de regressão tipo Cox é dada por

$$
h(t \mid x)=\left\{a \phi_{0,0}(t)+\sum_{j=0}^{J} \sum_{k=0}^{2^{j}-1} b_{j, k} \psi_{j, k}(t)\right\} \exp \left[x\left\{c \phi_{0,0}(t)+\sum_{j=0}^{J} \sum_{k=0}^{2^{j}-1} d_{j, k} \psi_{j, k}(t)\right\}\right],
$$

em que $a, b_{0,0}, \ldots, b_{J, 2^{J}-1}, c, d_{0,0}, \ldots, d_{J, 2^{J}-1}$ são os coeficientes a serem estimados.

Observe se os coeficientes de detalhes, $d_{0,0}, \ldots, d_{J, 2^{J}-1}$, são todos nulos, obtemos a proporcionalidade dos riscos para uma dada covariável. Logo, representar as funções $h_{0}(\cdot)$ e $\beta_{0}(\cdot)$ via ondaletas de Haar possui algumas vantagens, como avaliar a adequação do pressuposto de proporcionalidade para uma dada covariável, por meio de testes de hipóteses; estimar o comportamento do efeito da covariável sobre a taxa de falha, assim como a função de risco basal em qualquer instante de tempo, além de predições da função de sobrevivência para um paciente específico.

\subsubsection{Estimação dos parâmetros}

Utilizamos o método de máxima verossimilhança para estimar os parâmetros do modelo (4.9), que é baseado na função verossimilhança (3.6). Seja $\theta=\left(a, b_{0,0}, \ldots, b_{J, 2^{J}-1}, c, d_{0,0}, \ldots, d_{J, 2^{J}-1}\right)^{\mathrm{T}} \in \mathbb{R}^{p}$ o vetor de parâmetros de dimensão $p=2^{J+2}$ a ser estimado. A função logverossimilhança de $\theta$ para estimar $h_{0}(t) \mathrm{e}$ $\beta_{0}(t)$ é expressa por

$$
\begin{aligned}
\ell(\theta)= & -\sum_{i=1}^{n} \int_{0}^{t_{i}}\left\{a \phi_{0,0}(s)+\sum_{j=0}^{J} \sum_{k=0}^{2^{j}-1} b_{j, k} \psi_{j, k}(s)\right\} \exp \left(x_{i}\left\{c \phi_{0,0}(s)+\sum_{j=0}^{J} \sum_{k=0}^{2^{j}-1} d_{j, k} \psi_{j, k}(s)\right\}\right) d s \\
& +\sum_{i=1}^{n} \delta_{i} \log \left\{a \phi_{0,0}\left(t_{i}\right)+\sum_{j=0}^{J} \sum_{k=0}^{2^{j}-1} b_{j, k} \psi_{j, k}\left(t_{i}\right)\right\}+\sum_{i=1}^{n} \delta_{i} x_{i}\left\{c \phi_{0,0}\left(t_{i}\right)+\sum_{j=0}^{J} \sum_{k=0}^{2^{j}-1} d_{j, k} \psi_{j, k}\left(t_{i}\right)\right\} .
\end{aligned}
$$

É importante destacar que as ondaletas de Haar possuem expressões analíticas explícitas e, além disso, são funções constantes em determinados subintervalos, tornando assim possível obter expressões analíticas para o primeiro termo do lado direito em (4.10), ao contrário das séries de Fourier que necessitam de métodos de integração numérica, como mencionado no Capítulo 3. 
Os estimadores de máxima verossimilhança são os valores de $\beta$ que maximizam a função logverossimilhança (4.10). Eles são encontrados resolvendo o sistema de equações

$$
\mathbf{U}(\theta)=\mathbf{0},
$$

em que $\mathbf{U}(\theta) \in \mathbb{R}^{p}$ denota o vetor escore e é representado por

$$
\mathbf{U}(\theta)=\left(\mathrm{U}_{1}(\theta), \mathbf{U}_{2}^{\mathrm{T}}(\theta), \mathrm{U}_{3}(\theta), \mathbf{U}_{4}^{\mathrm{T}}(\theta)\right)^{\mathrm{T}}
$$

Especificamente,

$$
\begin{gathered}
\mathrm{U}_{1}(\theta)=\frac{\partial \ell(\theta)}{\partial a}, \quad \mathbf{U}_{2}(\theta)=\left(\frac{\partial \ell(\theta)}{\partial b_{0,0}}, \ldots, \frac{\partial \ell(\theta)}{\partial b_{J, 2^{J}-1}}\right)^{\mathrm{T}} \in \mathbb{R}^{p / 2-1} \\
\mathrm{U}_{3}(\theta)=\frac{\partial \ell(\theta)}{\partial c} \quad \text { e } \quad \mathbf{U}_{4}(\theta)=\left(\frac{\partial \ell(\theta)}{\partial d_{0,0}}, \ldots, \frac{\partial \ell(\theta)}{\partial d_{J, 2^{J}-1}}\right)^{\mathrm{T}} \in \mathbb{R}^{p / 2-1},
\end{gathered}
$$

sendo que

$$
\begin{aligned}
& \frac{\partial \ell(\theta)}{\partial a}=\sum_{i=1}^{n} \frac{\delta_{i} \phi_{0,0}\left(t_{i}\right)}{h_{0}\left(t_{i}\right)}-\sum_{i=1}^{n} \int_{0}^{t_{i}} \phi_{0,0}(s) \exp \left(x_{i} \beta_{0}(s)\right) d s, \\
& \frac{\partial \ell(\theta)}{\partial b_{j, k}}=\sum_{i=1}^{n} \frac{\delta_{i} \psi_{j, k}\left(t_{i}\right)}{h_{0}\left(t_{i}\right)}-\frac{\partial}{\partial b_{j, k}} \sum_{i=1}^{n} \int_{0}^{t_{i}} h_{0}(s) \exp \left(x_{i} \beta_{0}(s)\right) d s, \\
& \frac{\partial \ell(\theta)}{\partial c}=\sum_{i=1}^{n} \delta_{i} x_{i} \phi_{0,0}\left(t_{i}\right)-\frac{\partial}{\partial c} \sum_{i=1}^{n} \int_{0}^{t_{i}} h_{0}(s) \exp \left(x_{i} \beta_{0}(s)\right) d s
\end{aligned}
$$

e

$$
\frac{\partial \ell(\theta)}{\partial d_{j, k}}=\sum_{i=1}^{n} \delta_{i} x_{i} \psi_{j, k}\left(t_{i}\right)-\frac{\partial}{\partial d_{j, k}} \sum_{i=1}^{n} \int_{0}^{t_{i}} h_{0}(s) \exp \left(x_{i} \beta_{0}(s)\right) d s
$$

para $j=0, \ldots, J ; k=0, \ldots, 2^{j}-1$.

É importante ressaltar que, em situações práticas em que o risco basal está próximo de 0 , ajustar o modelo (4.9) aos dados pode ser complicado, no sentido de defrontar com problemas na otimização dos parâmetros. Em circunstâncias em que problemas como esses ocorrem, propomos como alternativa estimar os parâmetros do modelo em duas etapas.

Para realizar tais etapas, considere $\theta=\left(\theta_{1}^{\mathrm{T}}, \theta_{2}^{\mathrm{T}}\right)^{\mathrm{T}}=\left(a, b_{0,0}, \ldots, b_{J, 2^{J}-1}, c, d_{0,0}, \ldots, d_{J, 2^{J}-1}\right)^{\mathrm{T}}$ o vetor de parâmetros, em que $\theta_{1}=\left(a, b_{0,0}, \ldots, b_{J, 2^{J}-1}\right)^{\mathrm{T}}$ e $\theta_{2}=\left(c, d_{0,0}, \ldots, d_{J, 2^{J}-1}\right)^{\mathrm{T}}$ representam os vetores de parâmetros da função de risco basal e do coeficiente dependente do tempo, respectivamente.

Na primeira etapa, o vetor $\theta_{2}$ é estimado por meio do método de máxima verossimilhança parcial, como descrito na Seção 4.1. Posteriormente, na segunda etapa estimamos o vetor $\theta_{1}$ pelo método de máxima verossimilhança. A função $h_{0}(\cdot)$ é estimada a partir da função de verossimilhança (4.10), na qual envolve apenas os parâmetros de interesse, em que é obtida substituindo o vetor de perturbação $\theta_{2}$ pela estimativa 
de máxima verossimilhança parcial $\widehat{\theta}_{2}$. A função logverossimilhança perfilada é expressa por

$$
\begin{aligned}
\ell\left(\theta_{1}\right) \propto & -\sum_{i=1}^{n} \int_{0}^{t_{i}}\left\{a \phi_{0,0}(s)+\sum_{j=0}^{J} \sum_{k=0}^{2^{j}-1} b_{j, k} \psi_{j, k}(s)\right\} \exp \left(x_{i}\left\{\widehat{c} \phi_{0,0}(s)+\sum_{j=0}^{J} \sum_{k=0}^{2^{j}-1} \widehat{d}_{j, k} \psi_{j, k}(s)\right\}\right) d s \\
& +\sum_{i=1}^{n} \delta_{i} \log \left\{a \phi_{0,0}\left(t_{i}\right)+\sum_{j=0}^{J} \sum_{k=0}^{2^{j}-1} b_{j, k} \psi_{j, k}\left(t_{i}\right)\right\}
\end{aligned}
$$

Sejam $\widehat{\theta}_{1}$ o vetor que maximiza a função logverossimilhança com respeito a $\theta_{1}$ e $\mathbf{U}\left(\theta_{1}\right) \in \mathbb{R}^{p / 2}$ o vetor escore. O estimador $\widehat{\theta}_{1}$ é solução da equação $\mathbf{U}\left(\theta_{1}\right)=\mathbf{0}$, em que

$$
\mathbf{U}\left(\theta_{1}\right)=\left(\mathrm{U}_{1}\left(\theta_{1}\right), \mathbf{U}_{2}^{\mathrm{T}}\left(\theta_{1}\right)\right)^{\mathrm{T}} .
$$

Especificamente,

$$
\mathrm{U}_{1}\left(\theta_{1}\right)=\frac{\partial \ell\left(\theta_{1}\right)}{\partial a} \quad \text { e } \quad \mathbf{U}_{2}\left(\theta_{1}\right)=\left(\frac{\partial \ell\left(\theta_{1}\right)}{\partial b_{0,0}}, \ldots, \frac{\partial \ell\left(\theta_{1}\right)}{\partial b_{J, 2^{J}-1}}\right)^{\mathrm{T}} \in \mathbb{R}^{p / 2-1}
$$

sendo que

$$
\frac{\partial \ell\left(\theta_{1}\right)}{\partial a}=\sum_{i=1}^{n} \frac{\delta_{i} \phi_{0,0}\left(t_{i}\right)}{h_{0}\left(t_{i}\right)}-\sum_{i=1}^{n} \int_{0}^{t_{i}} \phi_{0,0}(s) \exp \left\{x_{i} \widehat{\beta}_{0}(s)\right\} d s
$$

$\mathrm{e}$

$$
\frac{\partial \ell\left(\theta_{1}\right)}{\partial b_{j, k}}=\sum_{i=1}^{n} \frac{\delta_{i} \psi_{j, k}\left(t_{i}\right)}{h_{0}\left(t_{i}\right)}-\frac{\partial}{\partial b_{j, k}} \sum_{i=1}^{n} \int_{0}^{t_{i}} h_{0}(s) \exp \left\{x_{i} \widehat{\beta}_{0}(s)\right\} d s
$$

para $j=0, \ldots, J ; k=0, \ldots, 2^{j}-1$.

Para encontrar as estimativas de máxima verossimilhança da função (4.10) ou (4.11) utilizamos métodos iterativos de maximização numérica. A construção de intervalos de confiança e testes de hipóteses para $\beta_{0}(t)$ e $h_{0}(t)$ são realizados supondo a normalidade assintótica dos estimadores de $\theta$. Logo, para um dado $t \in[0,1)$, as estimativas da variância de $\widehat{\beta}_{0}(\cdot)$ e $\widehat{h}_{0}(\cdot)$ são, respectivamente,

$$
\begin{aligned}
\widehat{\operatorname{var}}\left\{\widehat{\beta}_{0}(t)\right\}= & \left\{\phi_{0,0}(t)\right\}^{2} \widehat{\operatorname{var}}(\widehat{c})+2 \phi_{0,0}(t) \sum_{j=0}^{J} \sum_{k=0}^{2^{j}-1} \psi_{j, k}(t) \widehat{\operatorname{cov}}\left(\widehat{c}, \widehat{d}_{j, k}\right) \\
& +\widehat{\operatorname{cov}}\left\{\sum_{j=0}^{J} \sum_{k=0}^{2^{j}-1} \widehat{d}_{j, k} \psi_{j, k}(t), \sum_{j=0}^{J} \sum_{k=0}^{2^{j}-1} \widehat{d_{j, k}} \psi_{j, k}(t)\right\}
\end{aligned}
$$

$\mathrm{e}$

$$
\begin{aligned}
\widehat{\operatorname{var}}\left\{\widehat{h}_{0}(t)\right\}= & \left\{\phi_{0,0}(t)\right\}^{2} \widehat{\operatorname{var}}(\widehat{a})+2 \phi_{0,0}(t) \sum_{j=0}^{J} \sum_{k=0}^{2^{j}-1} \psi_{j, k}(t) \widehat{\operatorname{cov}}\left(\widehat{a}, \widehat{b}_{j, k}\right) \\
& +\widehat{\operatorname{cov}}\left\{\sum_{j=0}^{J} \sum_{k=0}^{2^{j}-1} \widehat{b}_{j, k} \psi_{j, k}(t), \sum_{j=0}^{J} \sum_{k=0}^{2^{j}-1} \widehat{b}_{j, k} \psi_{j, k}(t)\right\}
\end{aligned}
$$

e fazendo o uso da normalidade assintótica dos estimadores, podemos construir um intervalo de confiança para o efeito da covariável sobre a taxa de falha e para o risco basal para $t$ prefixado. As estimativas das variâncias e das covariâncias nas expressões acima são obtidas a partir da inversa da matriz de informação 
observada dada no Apêndice B.

\subsubsection{Testes de hipóteses: riscos proporcionais e/ou risco basal constante}

O modelo tipo Cox proposto em (4.9) é bastante flexível possibilitando incorporar riscos não proporcionais para uma dada covariável. Em situações práticas, é interessante verificar se a proporcionalidade dos riscos se sustenta, assim como verificar se o risco basal é constante ao longo tempo.

No cenário em que as estimativas dos parâmetros são obtidas em apenas uma etapa, considere o vetor de parâmetros $\theta=\left(\theta_{1}, \theta_{2}^{\mathrm{T}}\right)^{\mathrm{T}}$, em que $\theta_{1}=c$ e $\theta_{2}=\left(d_{0,0}, \ldots, d_{J, 2^{J}-1}\right)^{\mathrm{T}} \in \mathbb{R}^{p / 2-1}$, se o interesse é avaliar, para uma dada covariável, a proporcionalidade dos riscos. Por outro lado, se o objetivo é testar a função de risco basal constante ao longo do tempo, tome $\theta_{1}=\left(a, c, d_{0,0}, \ldots, d_{J, 2^{J}-1}\right)^{\mathrm{T}} \in \mathbb{R}^{p / 2+1}$ e $\theta_{2}=\left(b_{0,0}, \ldots, b_{J, 2^{J}-1}\right)^{\mathrm{T}} \in$ $\mathbb{R}^{p / 2-1}$. Em ambos os casos, a hipótese nula a ser testada é $H_{0}: \theta_{2}=\mathbf{0}$.

Em circunstâncias em que o procedimento de estimação em duas etapas é utilizado, testar as hipóteses de interesse segue de forma análoga à Seção 3.3.2. Independente do método de estimação considerado, os três testes mencionados na Seção 3.3.2 são utilizados. As expressões dos elementos da matriz de informação observada, $\mathbf{I}(\theta)$, são apresentadas no Apêndice B.

\subsubsection{Predição da função de sobrevivência}

Após estimar o efeito da covariável sobre a taxa de falha e conhecer o comportamento do risco basal, temos ainda curiosidade em obter um estimador da função de sobrevivência condicional para um específico indivíduo.

Antes de propor um estimador para a função de sobrevivência, considere os vetores $\widehat{\mathbf{b}}=\left(\widehat{b}_{1}, \ldots, \widehat{b}_{p^{*}}\right)^{\mathrm{T}} \in$ $\mathbb{R}^{p^{*}}$ e $\widehat{\mathbf{r}}=\left(\widehat{r}_{1}, \ldots, \widehat{r}_{p^{*}}\right)^{\mathrm{T}} \in \mathbb{R}^{p^{*}}$, de modo que

$$
\widehat{b}_{k}=\widehat{\beta}_{0}(t) \mathbb{I}_{\left\{(k-1) / p^{*} \leq t<k / p^{*}\right\}} \quad \text { e } \quad \widehat{r}_{k}=\widehat{h}_{0}(t) \mathbb{I}_{\left\{(k-1) / p^{*} \leq t<k / p^{*}\right\}}, \quad k=1, \ldots, p^{*}=2^{J+1},
$$

em que $p^{*}$ representa o número de subdivisões do intervalo unitário, enquanto $\widehat{h}_{0}(\cdot)$ e $\widehat{\beta}_{0}(\cdot)$ são os estimadores de máxima verossimilhança/parcial da função de risco basal e do coeficiente dependente do tempo, respectivamente.

Utilizando essa notação, os estimadores de $\widehat{\beta}_{0}(\cdot)$ e $\widehat{h}_{0}(\cdot)$ são, respectivamente, reescritos na forma

$$
\widehat{\beta}_{0}(t)=\sum_{k=1}^{p^{*}} \widehat{b}_{k} \mathbb{I}_{\left\{(k-1) / p^{*} \leq t<k / p^{*}\right\}} \text { e } \quad \widehat{h}_{0}(t)=\sum_{k=1}^{p^{*}} \widehat{r}_{k} \mathbb{I}_{\left\{(k-1) / p^{*} \leq t<k / p^{*}\right\}} .
$$

Desse modo, propomos estimar a função de risco basal acumulada por

$$
\widehat{H}_{0}(t)=\frac{1}{p^{*}} \sum_{k=2}^{p^{*}} \sum_{j=1}^{k-1} \widehat{r}_{j} \mathbb{I}_{\left\{(k-1) / p^{*} \leq t<k / p^{*}\right\}}+\sum_{k=1}^{p^{*}}\left\{t-\left(\frac{k-1}{p^{*}}\right)\right\} \widehat{r}_{k} \mathbb{I}_{\left\{(k-1) / p^{*} \leq t<k / p^{*}\right\}},
$$

e assim, o estimador da função de sobrevivência basal é

$$
\widehat{S}_{0}(t)=\exp \left(-\frac{1}{p^{*}} \sum_{k=2}^{p^{*}} \sum_{j=1}^{k-1} \widehat{r}_{j} \mathbb{I}_{\left\{(k-1) / p^{*} \leq t<k / p^{*}\right\}}-\sum_{k=1}^{p^{*}}\left\{t-\left(\frac{k-1}{p^{*}}\right)\right\} \widehat{r}_{k} \mathbb{I}_{\left\{(k-1) / p^{*} \leq t<k / p^{*}\right\}}\right) .
$$


Combinando as funções em (4.12), o risco condicional é dado por

$$
\widehat{h}(t \mid x)=\sum_{k=1}^{p^{*}} \widehat{r}_{k} \exp \left(x \widehat{b}_{k}\right) \mathbb{I}_{\left\{(k-1) / p^{*} \leq t<k / p^{*}\right\}} .
$$

Logo, para um específico valor da covariável $x$ e após algumas manipulações algébricas, propomos estimar o risco condicional acumulado por

$$
\widehat{H}(t \mid x)=\frac{1}{p^{*}} \sum_{k=2}^{p^{*}} \sum_{j=1}^{k-1} \widehat{r}_{j} \exp \left(x \widehat{b}_{k}\right) \mathbb{I}_{\left\{(k-1) / p^{*} \leq t<k / p^{*}\right\}}+\sum_{k=1}^{p^{*}}\left\{t-\left(\frac{k-1}{p^{*}}\right)\right\} \widehat{r}_{k} \exp \left(x \widehat{b}_{k}\right) \mathbb{I}_{\left\{(k-1) / p^{*} \leq t<k / p^{*}\right\}},
$$

e, consequentemente, a predição da função de sobrevivência para um indivíduo específico é

$$
\widehat{S}(t \mid x)=\exp \left[-\frac{1}{p^{*}} \sum_{k=2}^{p^{*}} \sum_{j=1}^{k-1} \widehat{r}_{j} \exp \left(\widehat{x}_{k}\right) \mathbb{I}_{\left\{(k-1) / p^{*} \leq t<k / p^{*}\right\}}-\sum_{k=1}^{p^{*}}\left\{t-\left(\frac{k-1}{p^{*}}\right)\right\} \widehat{r}_{k} \exp \left(\widehat{x}_{k}\right) \mathbb{I}_{\left\{(k-1) / p^{*} \leq t<k / p^{*}\right\}}\right] .
$$

Observamos que devido à função de risco basal ser representada via bases de ondaletas de Haar, a predição da função de sobrevivência possui expressão analítica simples dependendo apenas dos parâmetros, ao contrário do que ocorre no cenário semiparamétrico, em que o estimador da função de sobrevivência condicional também depende do estimador de Breslow generalizado.

É importante ainda mencionar que a estimação das funções de risco basal e condicional pode ser obtida em qualquer instante de tempo no intervalo unitário, diferentemente do cenário semiparamétrico, em que a estimação dessas funções está restrita aos $D$ tempos distintos de falha.

Fazendo uma comparação das abordagens de estimação por meio de ondaletas de Haar e séries de Fourier, podemos destacar que as bases de ondaletas de Haar levam vantagem em relação às bases de Fourier, no sentido de apresentarem expressões analíticas explícitas para o estimador da função de sobrevivência condicional. Além disso, as ondaletas costumam levar vantagem, visto que elas são localizadas em tempo e frequência, simultaneamente, ao contrário das séries de Fourier, que são localizadas apenas em frequência. Por outro lado, adotar as bases de Fourier pode ser uma vantagem, pois as curvas estimadas serão sempre contínuas e suaves, ao contrário das ondaletas de Haar que apresentam pontos de descontinuidade. 


\section{Capítulo 5}

\section{Simulações}

O presente capítulo tem a finalidade de avaliar os métodos inferenciais propostos nos Capítulos 3 e 4 . Para isso, realizamos diversos estudos de simulação em que amostras foram geradas e posteriormente os modelos discutidos foram ajustados. As estimativas dos parâmetros foram utilizadas para estimar medidas como a raiz quadrada do erro quadrático médio (REQM) e o erro padrão (EP). Além disso, após estimar o coeficiente dependente do tempo, escolhemos o valor ótimo de $J$ a partir do erro quadrático médio e também pelos critérios de seleção AIC e BIC.

Os procedimentos de estimação via bases de Fourier e ondaletas de Haar também são comparados no sentido de identificar qual abordagem fornece o melhor ajuste e ainda qual deles produz o menor erro quadrático médio.

Este capítulo apresenta em tabelas o resumo dos resultados das simulações e em gráficos as estimativas da função de densidade das estimativas, QQ plot das estimativas dos parâmetros, assim como as estimativas médias do coeficiente dependente do tempo obtidas por meio de bases de Fourier e ondaletas de Haar.

Para estimar os parâmetros dos modelos, desenvolvemos programas computacionais na linguagem $\mathrm{R}$ (R Core Team, 2014), bem como uma rotina para a geração de amostras, que foram utilizadas para avaliar o desempenho do processo de estimação. Os procedimentos de geração das amostras são apresentados no Apêndice A.

\subsection{Estudo 1}

Neste primeiro estudo, adotamos como verdadeiro efeito da covariável sobre a taxa de falha uma expansão em séries de ondaletas de Haar com o nível de resolução $J=0$, de tal modo que o coeficiente dependente do tempo é dado por

$$
\beta_{0}(t)=c \phi_{0,0}(t)+d \psi_{0,0}(t)
$$

Essa função pode ser reescrita como

$$
\beta_{0}(t)=\left\{\begin{array}{rc}
c+d, & \text { se } 0 \leq t<0,5, \\
c-d, & \text { se } 0,5 \leq t<1, \\
0, & \text { caso contrário. }
\end{array}\right.
$$

Em cada amostra simulada, geramos uma única covariável, de forma balanceada, a fim de classificar as unidades amostrais em dois grupos, que podem ser denominados controle e tratamento. Além disso, 
adotamos risco basal constante, $h_{0}(t)=0,15$ e fixamos $c=d=1$. Os tempos de censura são gerados de uma distribuição uniforme $\mathrm{U}(0, \tau)$, em que $\tau$ é selecionada para gerar a proporção de censura desejada. Optamos por tamanho de amostras iguais a $n \in\{128,256,512,1024\}$, com proporções de censuras de $0 \%$, $10 \%$ e $30 \%$. Neste cenário simulado, estamos interessados apenas no efeito da covariável sobre a taxa de falha, assim o modelo de regressão tipo Cox (4.1) com nível de resolução $J=0$ é ajustado a fim de obter as estimativas dos parâmetros.

Para cada conjunto de parâmetros, um total de 1000 amostras aleatórias foram geradas, e para cada configuração obtemos a média das estimativas de máxima verossimilhança parcial (via ondaletas clássicas) e com as estimativas dos parâmetros estimamos o REQM e EP (assintótico). Além disso, obtemos a média dos desvios padrão (DP) das estimativas, amplitudes dos intervalos de confiança de 95\%, assim como a probabilidade de cobertura (PC) do intervalo de confiança bilateral de $95 \%$.

A Tabela 5.1 apresenta as medidas descritivas dos estimadores do efeito dependente do tempo. Os resultados mostram, como esperado, que à medida que o tamanho amostral aumenta, as médias das estimativas se aproximam dos valores verdadeiros; à medida que a proporção de censura na amostra aumenta, em média, o erro quadrático médio, o erro padrão, o desvio padrão e a amplitude aumentam para um dado tamanho amostral. Observe também que o REQM, o erro padrão e o desvio padrão ficam próximos conforme o tamanho da amostra aumenta. Vale ainda ressaltar que a probabilidade de cobertura dos intervalos de confiança de 95\% para ambos os parâmetros se aproxima bem do nível nominal esperado.

Tabela 5.1: Resultados das simulações como função do tamanho amostral e da proporção de censura na amostra.

\begin{tabular}{|c|c|c|c|c|c|c|c|c|}
\hline$n$ & Censura & Parâmetro & Estimativa & REQM & EP & DP & Amplitude & $\mathrm{PC}$ \\
\hline \multirow{6}{*}{128} & \multirow{2}{*}{$0 \%$} & $c$ & 1,037 & 0,736 & 1,178 & 0,735 & 4,616 & 0,971 \\
\hline & & $d$ & 1,077 & 0,729 & 1,178 & 0,726 & 4,616 & 0,975 \\
\hline & \multirow{2}{*}{$10 \%$} & $c$ & 1,049 & 0,746 & 1,223 & 0,745 & 4,793 & 0,974 \\
\hline & & $d$ & 1,066 & 0,753 & 1,223 & 0,750 & 4,793 & 0,978 \\
\hline & \multirow{2}{*}{$30 \%$} & $c$ & 0,987 & 0,802 & 1,498 & 0,802 & 5,871 & 0,969 \\
\hline & & $d$ & 1,114 & 0,799 & 1,498 & 0,791 & 5,871 & 0,979 \\
\hline \multirow{6}{*}{256} & \multirow{2}{*}{$0 \%$} & $c$ & 1,025 & 0,373 & 0,354 & 0,371 & 1,390 & 0,955 \\
\hline & & $d$ & 1,037 & 0,382 & 0,354 & 0,380 & 1,390 & 0,947 \\
\hline & \multirow{2}{*}{$10 \%$} & $c$ & 1,027 & 0,377 & 0,357 & 0,376 & 1,400 & 0,963 \\
\hline & & $d$ & 1,037 & 0,383 & 0,357 & 0,382 & 1,400 & 0,947 \\
\hline & \multirow{2}{*}{$30 \%$} & $c$ & 1,025 & 0,379 & 0,363 & 0,379 & 1,421 & 0,962 \\
\hline & & $d$ & 1,038 & 0,389 & 0,363 & 0,387 & 1,421 & 0,947 \\
\hline \multirow{6}{*}{512} & \multirow{2}{*}{$0 \%$} & $c$ & 1,007 & 0,251 & 0,242 & 0,251 & 0,947 & 0,952 \\
\hline & & $d$ & 1,016 & 0,259 & 0,242 & 0,259 & 0,947 & 0,939 \\
\hline & \multirow{2}{*}{$10 \%$} & $c$ & 1,007 & 0,253 & 0,243 & 0,253 & 0,953 & 0,950 \\
\hline & & $d$ & 1,017 & 0,261 & 0,243 & 0,260 & 0,953 & 0,948 \\
\hline & \multirow{2}{*}{$30 \%$} & $c$ & 1,007 & 0,255 & 0,246 & 0,254 & 0,966 & 0,953 \\
\hline & & $d$ & 1,017 & 0,263 & 0,246 & 0,263 & 0,966 & 0,944 \\
\hline \multirow{6}{*}{1024} & \multirow{2}{*}{$0 \%$} & $c$ & 1,000 & 0,173 & 0,167 & 0,174 & 0,654 & 0,947 \\
\hline & & $d$ & 1,005 & 0,167 & 0,167 & 0,166 & 0,654 & 0,949 \\
\hline & \multirow{2}{*}{$10 \%$} & $c$ & 1,000 & 0,176 & 0,168 & 0,175 & 0,658 & 0,945 \\
\hline & & $d$ & 1,004 & 0,167 & 0,168 & 0,167 & 0,658 & 0,948 \\
\hline & \multirow{2}{*}{$30 \%$} & $c$ & 1,001 & 0,176 & 0,170 & 0,176 & 0,666 & 0,955 \\
\hline & & $d$ & 1,004 & 0,170 & 0,170 & 0,170 & 0,666 & 0,947 \\
\hline
\end{tabular}


As Figuras 5.1 a 5.4 apresentam esboços das densidades estimadas dos estimadores do modelo, obtidas de 1000 amostras simuladas de tamanho 256 e 1024 contendo $0 \%$ e 30\% de censura, respectivamente. A linha tracejada indica o valor verdadeiro na geração dos dados e a linha pontilhada a curva teórica da distribuição normal. É possível observar que as estimativas estão distribuídas em torno dos valores verdadeiros utilizados na geração das amostras simuladas, indicando não haver vícios consideráveis, como podemos observar na Tabela 5.1. Obsrvamos ainda que as formas das densidades estimadas e as curvas teóricas sugerem uma aproximação assintótica para a distribuição normal. Além disso, nas Figuras 5.5 a 5.8 apresentamos os gráficos QQ plot com envelope simulado das estimativas dos parâmetros. No cenário em que o tamanho da amostra é pequeno $(n=256)$ observamos, pelo gráfico, que há indícios de uma ligeira fuga da normalidade para $d$, independentemente do nível de censura na amostra. Por outro lado, quando o tamanho da amostra é grande $(n=1024)$ não há evidências de afastamentos da normalidade, sugerindo que os resultados conhecidos para os estimadores de máxima verossimilhança também se aplica ao nosso modelo.
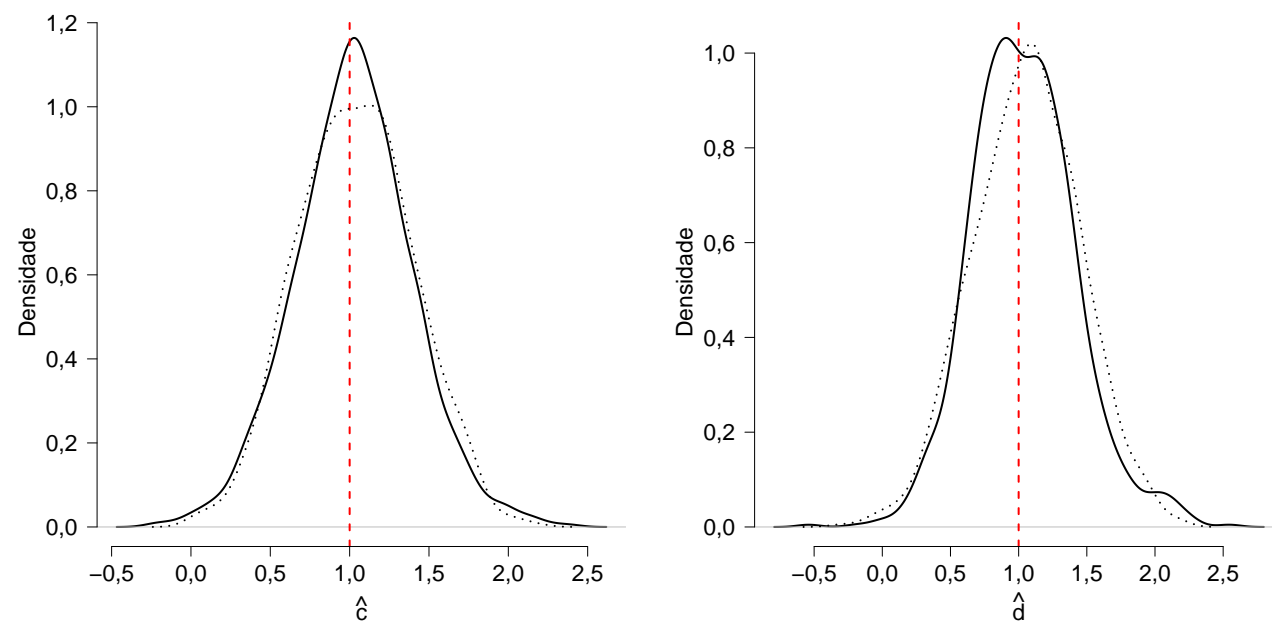

Figura 5.1: Estimativa da função densidade das estimativas do coeficiente da regressão (linha contínua) e a curva teórica normal (linha pontilhada) para $n=256$ e $0 \%$ de censura.
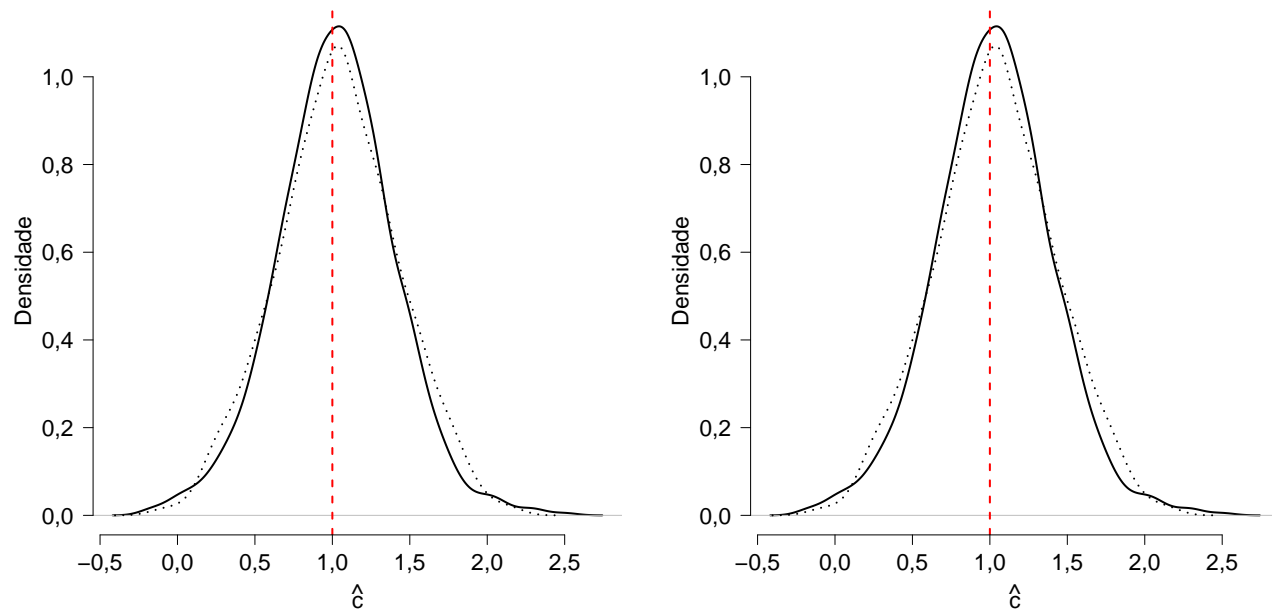

Figura 5.2: Estimativa da função densidade das estimativas do coeficiente da regressão (linha contínua) e a curva teórica normal (linha pontilhada) para $n=256$ e $30 \%$ de censura. 

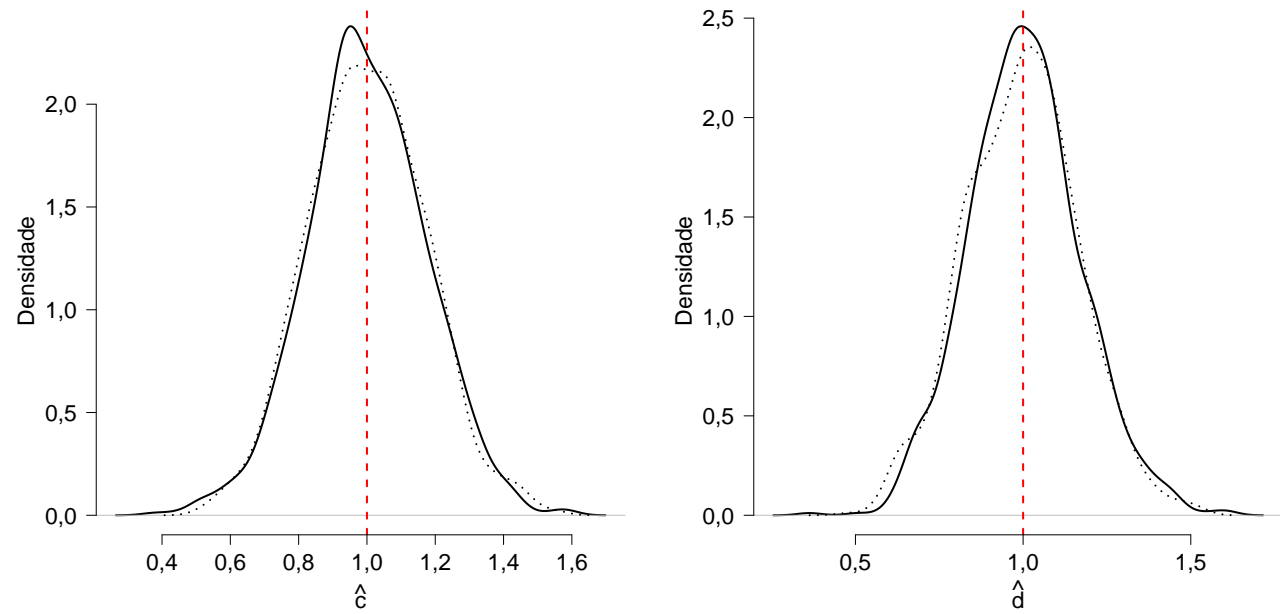

Figura 5.3: Estimativa da função densidade das estimativas do coeficiente da regressão (linha contínua) e a curva teórica normal (linha pontilhada) para $n=1024$ e $0 \%$ de censura.
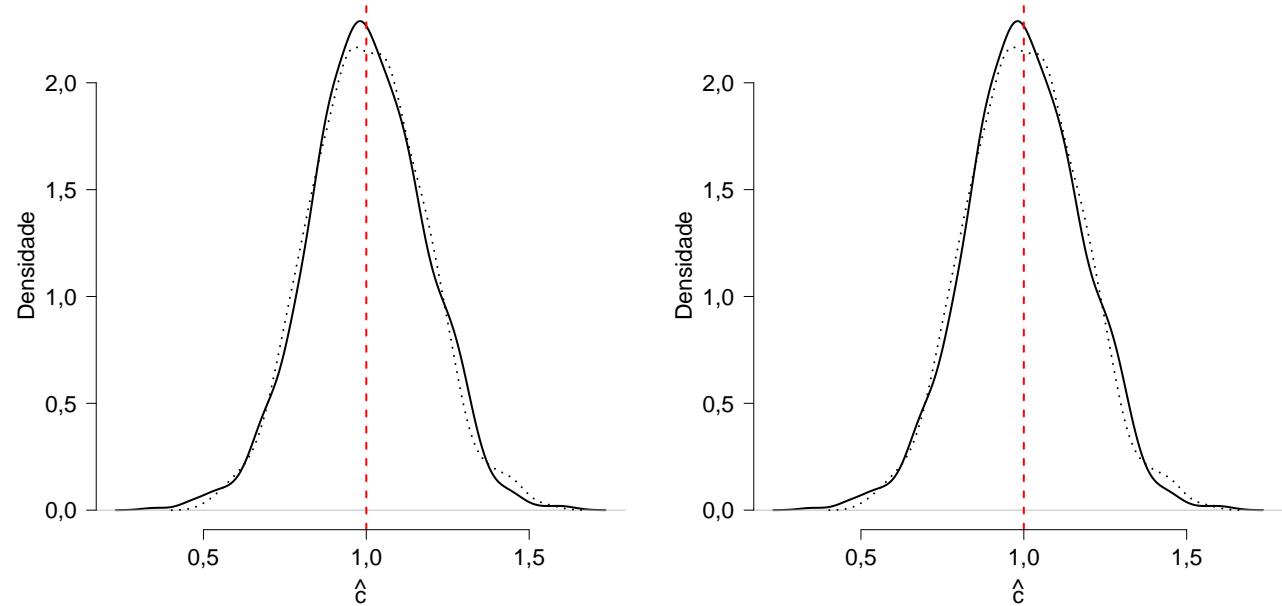

Figura 5.4: Estimativa da função densidade das estimativas do coeficiente da regressão (linha contínua) e a curva teórica normal (linha pontilhada) para $n=1024$ e 30\% de censura. 


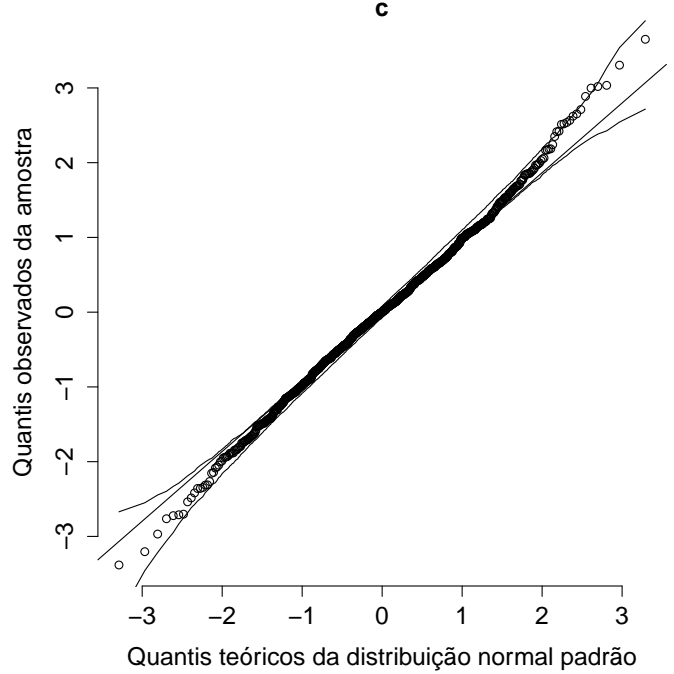

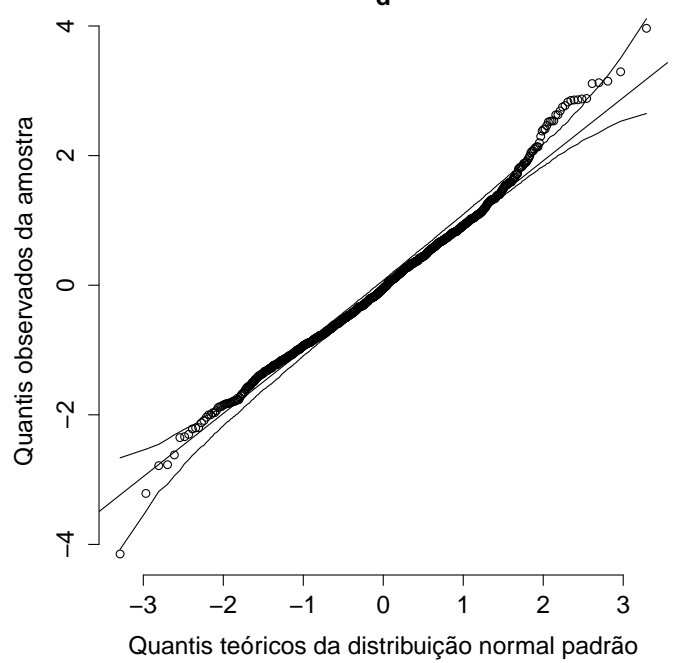

Figura 5.5: $Q Q$ plot das estimativas dos parâmetros para $n=256$ e $0 \%$ de censura.
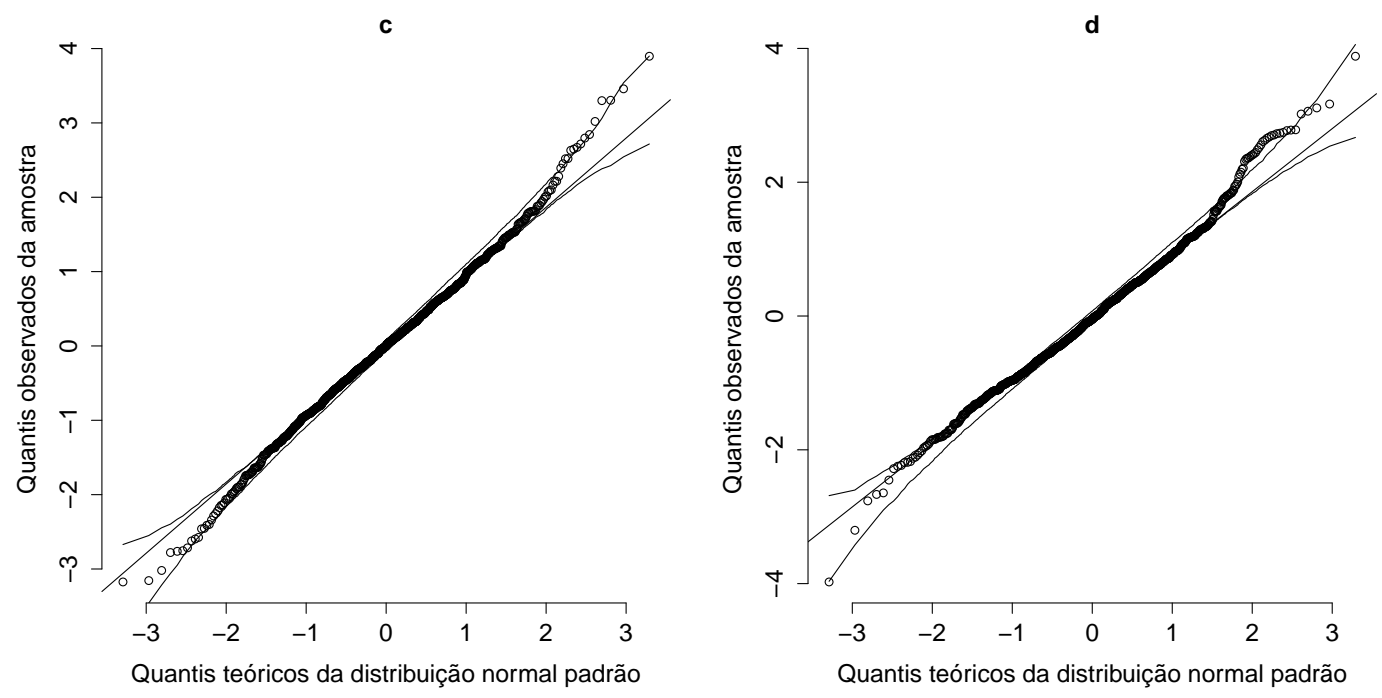

Figura 5.6: $Q Q$ plot das estimativas dos parâmetros $n=256$ e $30 \%$ de censura. 

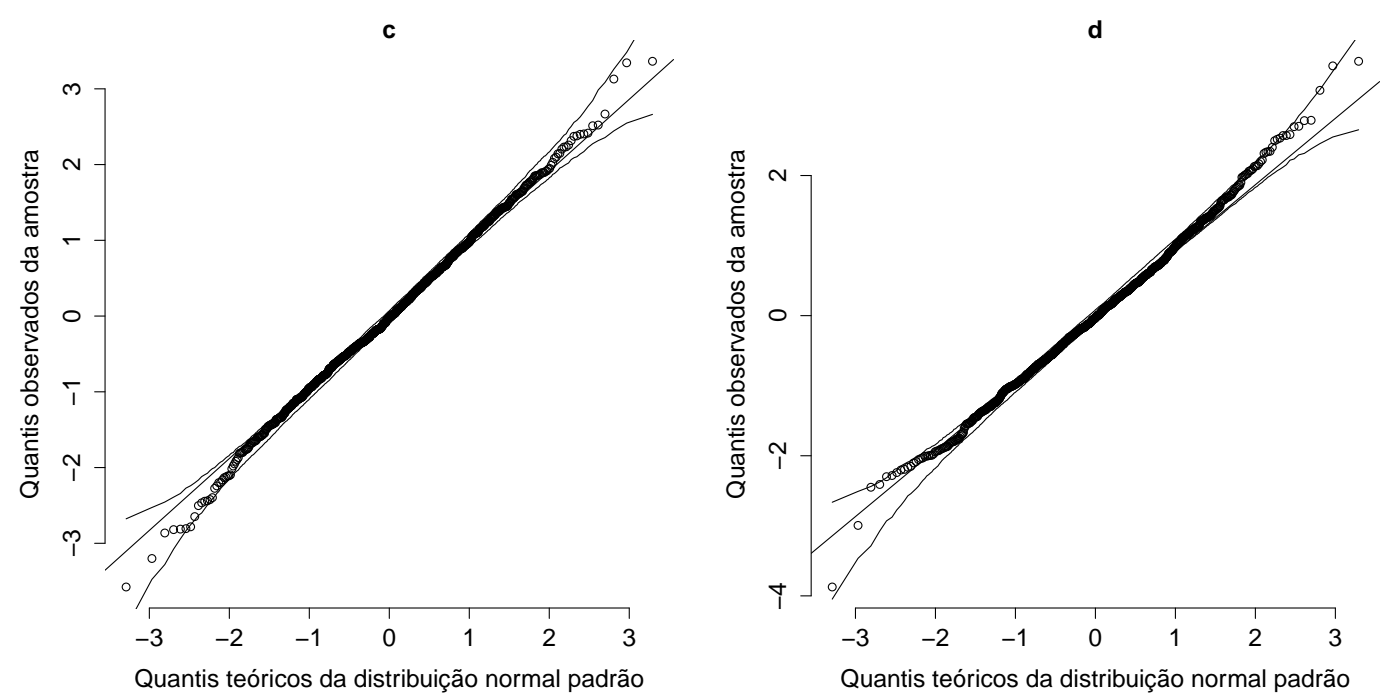

Figura 5.7: $Q Q$ plot das estimativas dos parâmetros para $n=1024$ e $0 \%$ de censura.
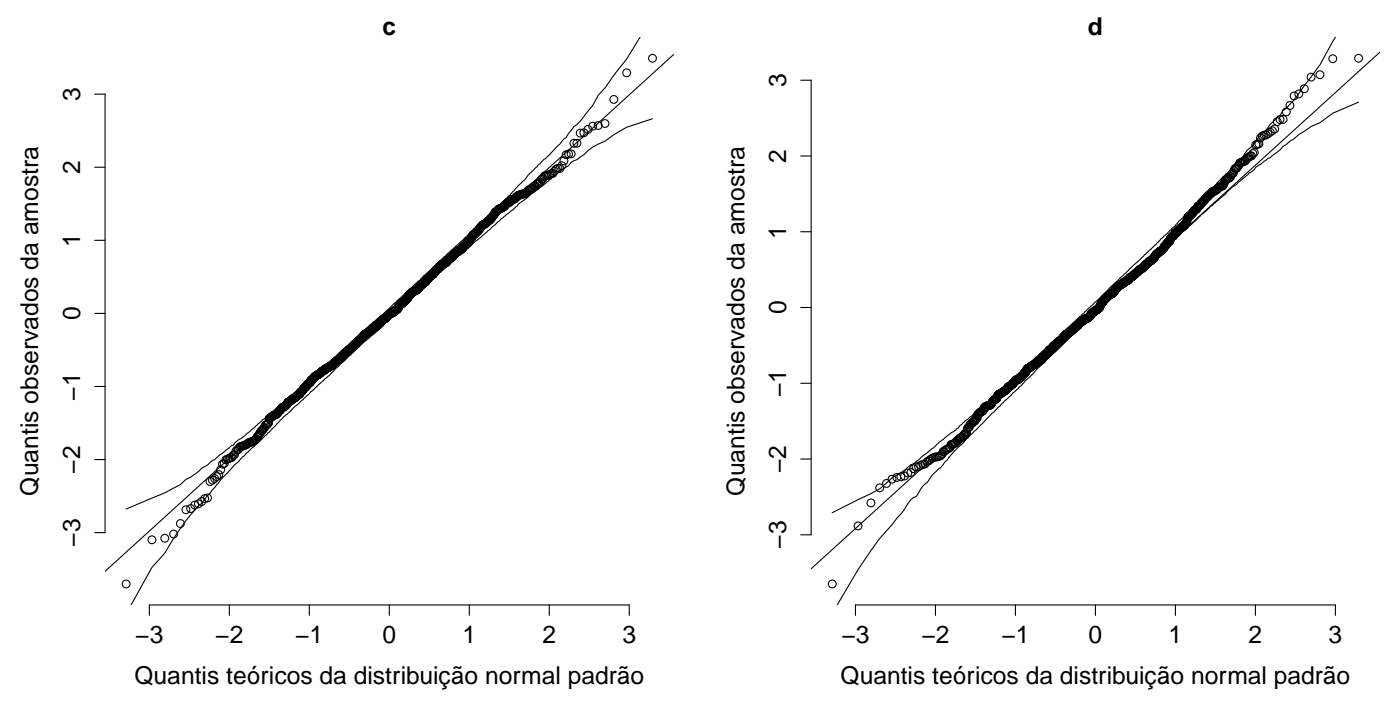

Figura 5.8: $Q Q$ plot das estimativas dos parâmetros para $n=1024$ e $30 \%$ de censura.

A performance da estatística de teste de Wald e da razão de verossimilhanças (RV) foi avaliada para o nível de significância nominal $\alpha=0,05$ com o objetivo de testar se o efeito da covariável sobre a taxa de falha é constante ao longo do tempo. Para realizar tal estudo, simulamos conjuntos de dados $\operatorname{com} c=1$, $d \in\{0 ; 0,01 ; 0,5 ; 1 ; 1,5\}, h_{0}(t)=0,15$ e diferentes tamanhos de amostras e percentuais de censura. Um total de 1000 amostras aleatórias foram geradas, e para cada configuração obtemos a taxa de rejeição da hipótese nula $\left(H_{0}: d=0\right)$. O tamanho e o poder dos testes são apresentados na Tabela 5.2.

Podemos concluir que, à medida que $d$ se aproxima de zero, menor é a taxa de rejeição da hipótese nula, como esperado. Por outro lado, conforme o tamanho amostral e $d$ aumentam, a taxa de rejeição para a hipótese nula também aumenta, independente da estatística do teste utilizada. Se a hipótese nula é verdadeira, observamos que para a estatística de Wald, a taxa de rejeição de $H_{0}$ fica abaixo do nível de significância nominal de 5\% para amostras pequenas (teste conservativo), mas converge para o tamanho esperado à medida que o tamanho da amostra fica maior. Ressaltamos ainda que o teste baseado na estatística RV fornece taxa de rejeição bem próximas de 0,05 , independente do tamanho amostral. 
Tabela 5.2: Taxas de rejeição da hipótese nula para diversos tamanhos de amostras e percentuais de censura.

\begin{tabular}{|c|c|c|c|c|c|c|c|c|c|c|c|c|c|}
\hline \multirow[b]{2}{*}{ Teste } & \multirow[b]{2}{*}{$d$} & \multicolumn{4}{|c|}{$0 \%$ censura } & \multicolumn{4}{|c|}{$10 \%$ censura } & \multicolumn{4}{|c|}{$30 \%$ censura } \\
\hline & & 128 & 256 & 512 & 1024 & 128 & 256 & 512 & 1024 & 128 & 256 & 512 & 1024 \\
\hline \multirow{5}{*}{$\frac{\pi}{\pi}$} & 0 & 0,022 & 0,046 & 0,041 & 0,043 & 0,023 & 0,048 & 0,039 & 0,043 & 0,020 & 0,048 & 0,044 & 0,051 \\
\hline & 0,01 & 0,022 & 0,048 & 0,043 & 0,043 & 0,023 & 0,050 & 0,043 & 0,044 & 0,020 & 0,050 & 0,043 & 0,048 \\
\hline & 0,5 & 0,193 & 0,393 & 0,642 & 0,916 & 0,192 & 0,385 & 0,641 & 0,915 & 0,190 & 0,383 & 0,640 & 0,901 \\
\hline & 1,0 & 0,546 & 0,860 & 0,990 & 1,000 & 0,543 & 0,852 & 0,989 & 1,000 & 0,530 & 0,848 & 0,989 & 1,000 \\
\hline & 1,5 & 0,693 & 0,943 & 0,994 & 1,000 & 0,680 & 0,942 & 0,996 & 1,000 & 0,662 & 0,940 & 0,995 & 0,999 \\
\hline \multirow{5}{*}{$\gtrsim$} & 0 & 0,042 & 0,054 & 0,045 & 0,045 & 0,041 & 0,055 & 0,046 & 0,046 & 0,041 & 0,056 & 0,047 & 0,055 \\
\hline & 0,01 & 0,054 & 0,054 & 0,044 & 0,044 & 0,052 & 0,054 & 0,045 & 0,045 & 0,052 & 0,058 & 0,046 & 0,054 \\
\hline & 0,5 & 0,226 & 0,401 & 0,641 & 0,915 & 0,222 & 0,391 & 0,638 & 0,914 & 0,220 & 0,392 & 0,638 & 0,900 \\
\hline & 1,0 & 0,579 & 0,858 & 0,986 & 1,000 & 0,576 & 0,849 & 0,983 & 1,000 & 0,568 & 0,845 & 0,984 & 1,000 \\
\hline & 1,5 & 0,772 & 0,976 & 1,000 & 1,000 & 0,775 & 0,974 & 1,000 & 1,000 & 0,750 & 0,969 & 1,000 & 1,000 \\
\hline
\end{tabular}

\subsection{Estudo 2}

O objetivo deste segundo estudo é avaliar a performance de $\widehat{\beta}_{0}(\cdot)$ por meio da medida REQM (raiz quadrada do erro quadrático médio) dada por

$$
\mathrm{REQM}=\left[n_{0}^{-1} \sum_{k=1}^{n_{0}}\left\{\widehat{\beta}_{0}\left(u_{k}\right)-\beta_{0}\left(u_{k}\right)\right\}^{2}\right]^{1 / 2}
$$

em que $u_{k}, k=1, \ldots, n_{0}$ corresponde a uma grade de pontos na qual a função $\beta_{0}(\cdot)$ é estimada.

Como consequência de (5.1), quanto menor o valor de REQM, maiores os indicativos de estimativas plausíveis do coeficiente variante ao longo do tempo, o que acarreta um melhor ajuste do modelo. Avaliamos a performance da REQM considerando os tempos observados como grade de pontos para estimar $\beta_{0}(\cdot)$.

Para realizar este estudo, os tempos de vida são gerados pelo modelo (3.4) com função de risco basal constante, $h_{0}(t)=1$, e o efeito da covariável sobre a taxa de falha é assumido ser uma função crescente e contínua, $\beta_{0}(t)=t^{1 / 2}$. Além disso, geramos uma única covariável, a qual assume os valores 0 e 1 , com iguais probabilidades. Os tempos de censura são gerados de uma distribuição uniforme $\mathrm{U}(0, \tau)$, em que o parâmetro $\tau$ é convenientemente escolhido para controlar a proporção de censura na amostra. Adotamos cinco diferentes tamanhos de amostra $n \in\{400,600,800,1000,5000\}$ e dois diferentes níveis de censura $0 \%$ e $30 \%$.

Como nosso objetivo é estimar o efeito da covariável ao longo do tempo e identificar qual valor de $J$ produz menores REQM, ajustamos os modelos de regressão tipo Cox usando as bases de Fourier e ondaletas, (3.10) e (4.1), respectivamente.

Assim, para cada conjunto de parâmetros, um total de 100 amostras aleatórias foram geradas, e para cada configuração obtemos a média amostral e o desvio padrão da REQM. Os resultados das simulações obtidas por meio de bases de Fourier e ondaletas de Haar são resumidas, respectivamente, nas Tabelas 5.3 e 5.4.

Independente da representação adotada (via séries de Fourier ou ondaletas Haar) e do valor $J$, as médias amostrais da REQM bem como os desvios padrão decrescem à medida que o tamanho da amostra aumenta, como esperado. Observamos ainda que para tamanho amostral fixado, maiores médias e desvios padrão das REQM são observados na presença de censura.

No cenário em que o efeito da covariável ao longo do tempo é estimado via bases de Fourier e tempos deformados, os resultados indicam que para $n \leq 1000$, menores médias amostrais e desvios padrão 
são obtidos para $J=1$, independentemente da proporção de censura na amostra. Por outro lado, quando $n=5000$ e $0 \%$ de censura, $J=2$ fornece o menor REQM, ao passo que na presença de censura, a menor média ocorre para $J=3$.

Utilizando as bases de ondaletas de Haar para estimar o coeficiente dependente do tempo, observamos que, para uma taxa de censura fixada e tamanho amostral $n \leq 800$, menores médias e desvios padrão das REQM ocorrem para o nível de resolução $J=0$, enquanto que para tamanhos de amostras maiores, menores médias são observadas para $J=1$. É importante ainda destacar que quando há $30 \%$ de censura na amostra, o procedimento de estimação via ondaletas clássicas fornece menores REQM em relação às ondaletas deformadas, independente do tamanho amostral. No cenário em que as observações são completas, as ondaletas deformadas apresentam menores REQM para $n \leq 800$, ao passo que para amostras maiores as menores REQM são obtidas via ondaletas clássicas.

Tabela 5.3: Bases de Fourier: Média amostral e desvio padrão das REQM obtidas em 100 réplicas para J =1,2,3 e tempos deformados.

\begin{tabular}{cccccc}
\hline & & \multicolumn{2}{c}{ 0\% censura } & \multicolumn{2}{c}{$30 \%$ censura } \\
\cline { 3 - 6 }$n$ & $J$ & Média & Desvio padrão & Média & Desvio padrão \\
\hline \multirow{3}{*}{400} & 1 & 0,258 & 0,035 & 0,302 & 0,063 \\
& 2 & 0,291 & 0,044 & 0,346 & 0,078 \\
& 3 & 0,338 & 0,057 & 0,387 & 0,082 \\
& 1 & 0,220 & 0,016 & 0,250 & 0,041 \\
600 & 2 & 0,239 & 0,022 & 0,282 & 0,043 \\
& 3 & 0,267 & 0,026 & 0,315 & 0,048 \\
& 1 & 0,221 & 0,021 & 0,253 & 0,040 \\
800 & 2 & 0,228 & 0,024 & 0,270 & 0,044 \\
& 3 & 0,249 & 0,030 & 0,287 & 0,046 \\
& 1 & 0,205 & 0,014 & 0,237 & 0,033 \\
1000 & 2 & 0,211 & 0,017 & 0,256 & 0,034 \\
& 3 & 0,229 & 0,021 & 0,270 & 0,035 \\
5000 & 1 & 0,179 & 0,004 & 0,204 & 0,014 \\
& 2 & 0,170 & 0,006 & 0,205 & 0,013 \\
& 3 & 0,172 & 0,007 & 0,201 & 0,014 \\
\hline
\end{tabular}

As Figuras 5.9 a 5.11 representam a evolução das estimativas médias para o efeito da covariável ao longo do tempo obtidas via séries de Fourier, ondaletas clássicas e deformadas, respectivamente, considerando amostra de tamanho 400 e 30\% de censura na amostra. Embora a amostra seja relativamente moderada, para dados de sobrevivência, as estimativas médias do coeficiente dependente do tempo concordam com a função verdadeira, principalmente quando estimação é realizada por meio das bases de ondaletas de Haar (Figuras 5.10 e 5.11). No entanto, é importante mencionar que o procedimento de estimação baseado nas ondaletas deformadas subestima a verdadeira função em relação as ondaletas clássicas.

Vale ainda destacar que devido às funções seno e cosseno serem periódicas (Figura 5.9) e também às poucas falhas no início do experimento, estimativas superestimadas de $\beta_{0}(t)$ são observadas. Por outro lado, em consequência de observações censuradas e poucas falhas no final dos tempos, estimativas subestimadas ocorrem para o efeito da covariável ao longo do tempo.

Por meio deste estudo de simulação, observamos que as bases de ondaletas de Haar levam vantagem em relação às bases de Fourier, no sentido de apresentar, em média, estimativas mais próximas da verdadeira função, em especial nos extremos dos tempos observados. 
Tabela 5.4: Bases de ondaletas clássicas/deformadas: Média amostral e desvio padrão das REQM obtidas em 100 réplicas para $J=0,1,2$.

\begin{tabular}{|c|c|c|c|c|c|}
\hline \multirow[b]{2}{*}{$n$} & \multirow[b]{2}{*}{$J$} & \multicolumn{2}{|c|}{$0 \%$ censura } & \multicolumn{2}{|c|}{$30 \%$ censura } \\
\hline & & Média & Desvio padrão & Média & Desvio padrão \\
\hline & 0 & $0,238 / 0,222$ & $0,032 / 0,030$ & $0,246 / 0,280$ & $0,036 / 0,052$ \\
\hline \multirow[t]{2}{*}{400} & 1 & $0,277 / 0,275$ & $0,047 / 0,056$ & $0,285 / 0,303$ & $0,048 / 0,059$ \\
\hline & 2 & $0,377 / 0,381$ & $0,074 / 0,118$ & $0,396 / 0,384$ & $0,082 / 0,091$ \\
\hline \multirow{4}{*}{600} & 0 & $0,211 / 0,192$ & $0,021 / 0,019$ & $0,206 / 0,248$ & $0,019 / 0,035$ \\
\hline & 1 & $0,222 / 0,213$ & $0,029 / 0,026$ & $0,226 / 0,262$ & $0,035 / 0,043$ \\
\hline & 2 & 0,297 / 0,293 & $0,042 / 0,048$ & $0,314 / 0,317$ & $0,052 / 0,056$ \\
\hline & 0 & $0,207 / 0,193$ & $0,022 / 0,021$ & $0,196 / 0,240$ & $0,018 / 0,030$ \\
\hline \multirow[t]{3}{*}{800} & 1 & 0,214 / 0,209 & $0,031 / 0,029$ & $0,204 / 0,252$ & $0,029 / 0,039$ \\
\hline & 2 & 0,264 / 0,279 & $0,036 / 0,053$ & 0,267 / 0,291 & $0,037 / 0,044$ \\
\hline & 0 & $0,194 / 0,180$ & $0,015 / 0,014$ & 0,184 / 0,240 & $0,013 / 0,026$ \\
\hline \multirow[t]{3}{*}{1000} & 1 & $0,186 / 0,194$ & $0,018 / 0,025$ & $0,182 / 0,235$ & $0,020 / 0,030$ \\
\hline & 2 & $0,233 / 0,256$ & $0,026 / 0,037$ & $0,238 / 0,266$ & $0,031 / 0,036$ \\
\hline & 0 & 0,177 / 0,162 & $0,005 / 0,005$ & $0,160 / 0,212$ & $0,003 / 0,010$ \\
\hline \multirow[t]{2}{*}{5000} & 1 & $0,140 / 0,156$ & $0,005 / 0,009$ & 0,117 / 0,192 & $0,004 / 0,011$ \\
\hline & 2 & $0,142 / 0,186$ & $0,008 / 0,015$ & $0,123 / 0,194$ & $0,007 / 0,014$ \\
\hline
\end{tabular}

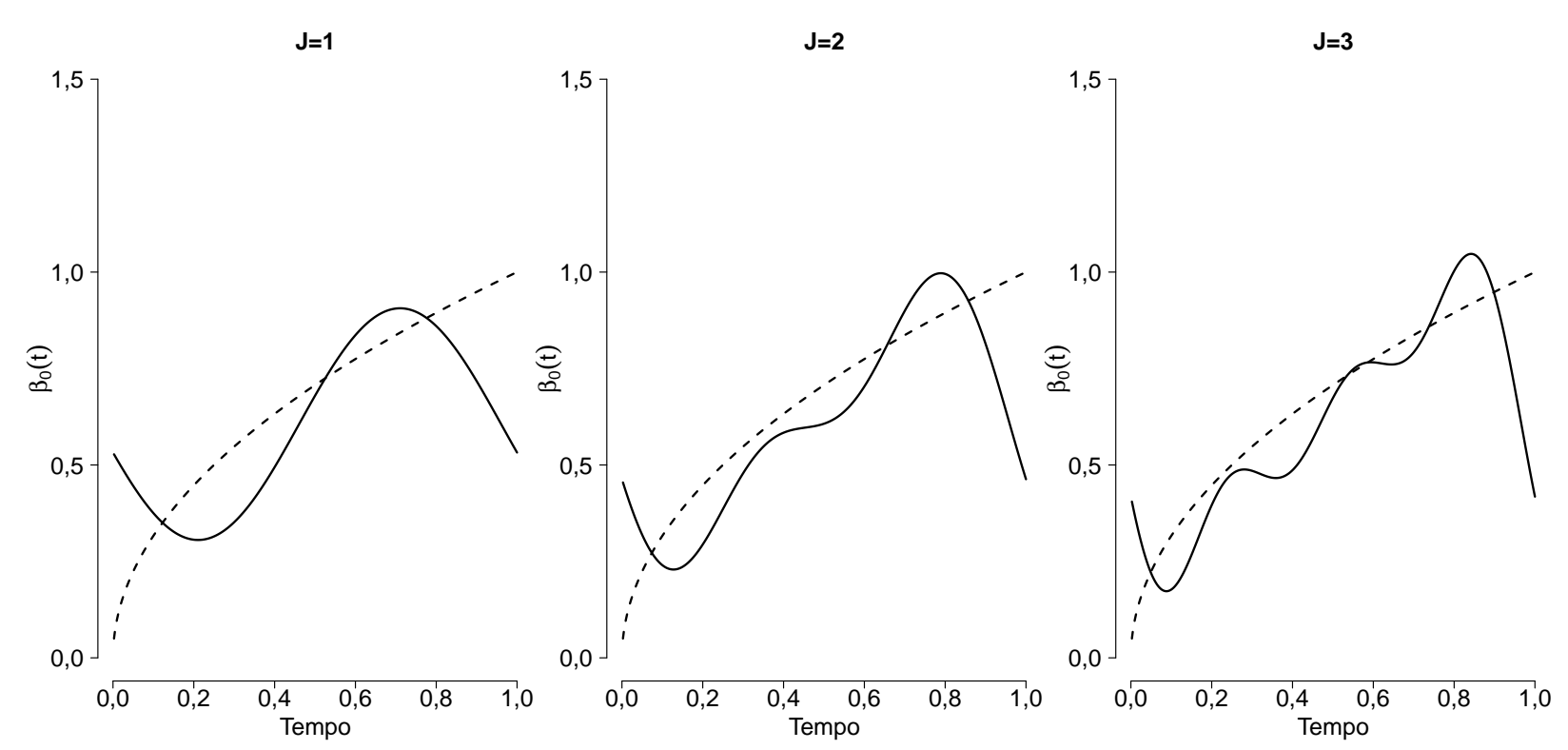

Figura 5.9: Séries de Fourier. Estimativas médias (linha contínua) de $\beta_{0}(t)$ juntamente com a verdadeira função (linha tracejada) para $n=400$ e $30 \%$ de censura e tempos deformados. 
$\mathrm{J}=0 \quad \mathrm{~J}=1 \quad \mathrm{~J}=2$
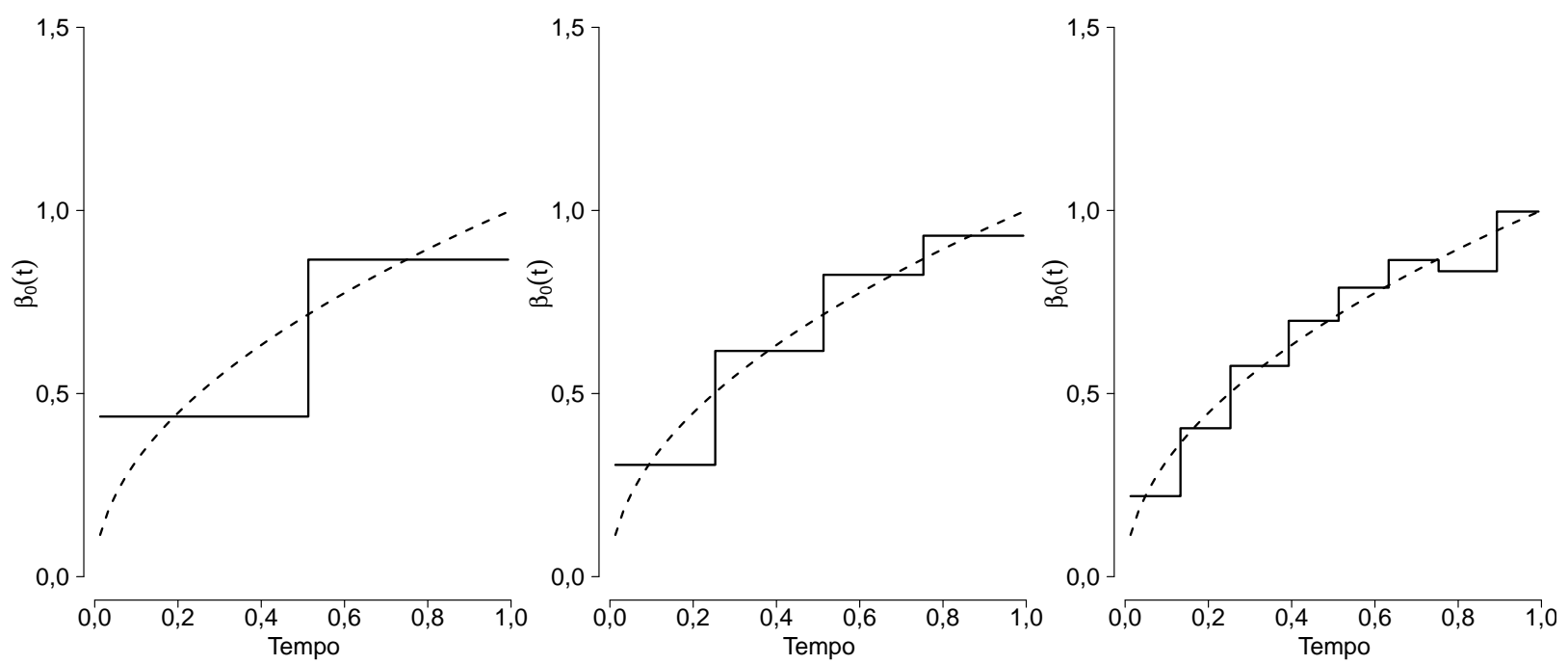

Figura 5.10: Ondaletas clássicas. Estimativas médias (linha contínua) de $\beta_{0}(t)$ juntamente com a verdadeira função (linha tracejada) para $n=400$ e $30 \%$ de censura.

$\mathrm{J}=0$

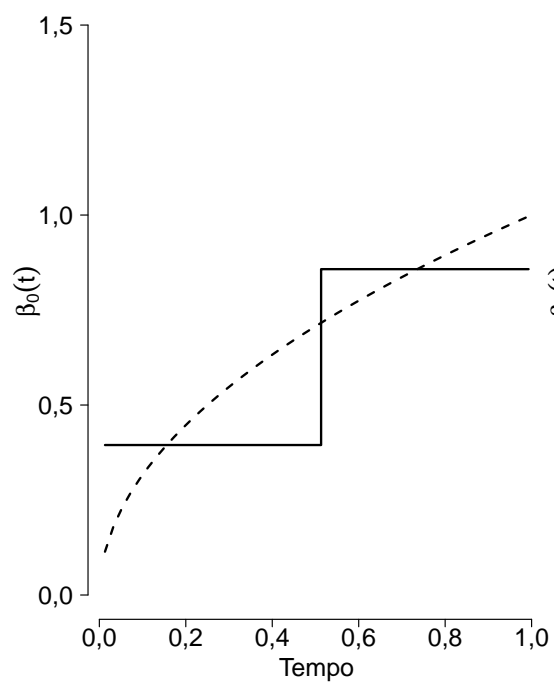

$\mathrm{J}=1$

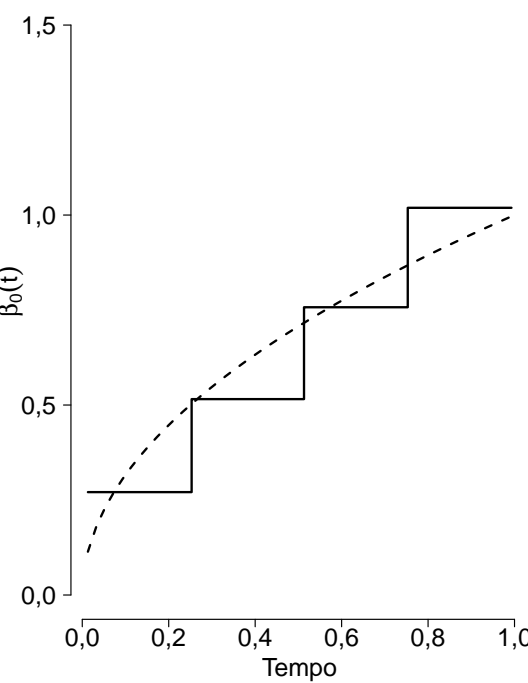

$\mathrm{J}=2$

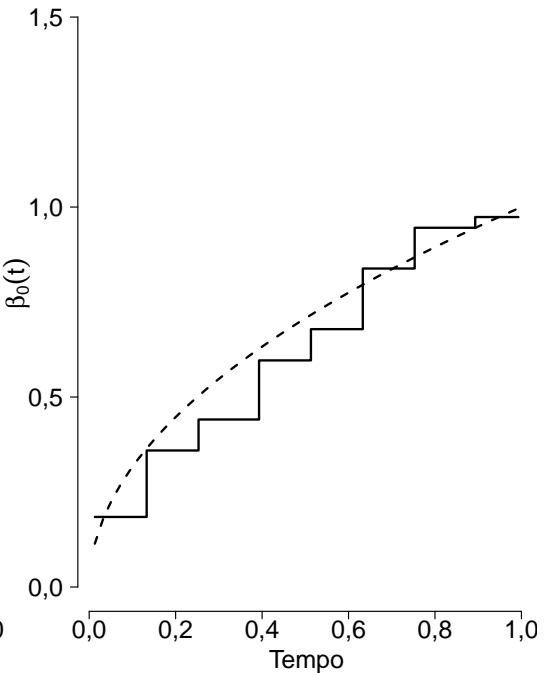

Figura 5.11: Ondaletas deformadas. Estimativas médias (linha contínua) de $\beta_{0}(t)$ juntamente com a verdadeira função (linha tracejada) para $n=400$ e $30 \%$ de censura. 


\subsection{Estudo 3}

Na literatura há diversos métodos para selecionar o valor ótimo de $J$ após estimar o coeficiente variante ao longo do tempo. Algumas ferramentas gráficas são a curva estimada ou o gráfico de resíduos, como mencionado em Huang e Shen (2004). Neste terceiro estudo de simulação, o valor de $J$ é escolhido de tal forma a minimizar uma função de critério apropriada, como abordado por Sleeper e Harrington (1990) e Abrahamowicz et al. (1996). Consideramos neste estudo dois critérios: critério de informação de Akaike (AIC) (Akaike, 1974) e o critério de informação bayesiano (BIC) (Schwarz, 1978). Esses critérios, respectivamente, consistem em escolher o valor de $J$ que minimiza os valores

$$
\mathrm{AIC}=-2 \ell(\widehat{\boldsymbol{\theta}})+2 p \quad \mathrm{e} \quad \mathrm{BIC}=-2 \ell(\widehat{\boldsymbol{\theta}})+p \log (n),
$$

em que $\widehat{\theta}$ é a estimativa de máxima verossimilhança ou máxima verossimilhança parcial e $p$ corresponde ao número de parâmetros estimados.

Uma vez que não é claro qual critério deve ser utilizado para a escolha do valor ótimo de $J$, conduzimos outro estudo de simulação a fim de comparar os critérios com respeito a $J$ e também em relação ao erro quadrático médio produzido ao utilizar esses critérios na escolha do valor de $J$.

Os conjuntos de dados simulados são obtidos como no estudo 2, em que os mesmos cenários são considerados e o efeito da covariável sobre a taxa de falha é assumido ser $\beta_{0}(t)=t^{1 / 2}$. Para cada configuração os modelos de regressão tipo Cox (3.10) e (4.1) foram ajustados e para cada critério de seleção foi obtido o valor mais adequado de $J$.

É importante mencionar que, embora tenhamos aplicado os critérios de informação para avaliar o valor de $J$ mais apropriado entre 0,1 ou 2 (bases de ondaletas de Haar), o nível de resolução $J=2$ não foi selecionado pelo critério BIC. Comportamento semelhante ocorre para as bases de Fourier, $J \in\{1,2,3\}$. Todavia, quando o tamanho da amostra é grande o nível $J=3$ é selecionado, como pode ser observado na Tabela 5.5. Os resultados indicam, considerando especialmente o critério BIC, que não são necessários valores elevados de $J$ para a estimação do efeito da covariável ao longo do tempo.

Por outro lado, se o critério de seleção AIC é escolhido, maiores valores de $J$ são selecionados à medida que o tamanho amostral aumenta, independente do procedimento de estimação utilizado. Além disso, para um tamanho de amostra fixado, o critério AIC parece priorizar valores de $J$ mais baixos conforme a taxa de censura aumenta.

Além da frequência em que cada nível ótimo $J$ foi escolhido em 100 repetições, apresentamos na Tabela 5.6 os valores médios (desvios padrão) do AIC e BIC para a transformação dos tempos observados pelo método deformado. Por meio dos valores médios de AIC e BIC é possível observar que a escolha do nível ótimo de $J$ (menor valor médio) apresenta comportamento semelhente aos resultados obtidos na Tabela 5.5. Observamos ainda que o desvio padrão dos critérios aumentam à medida que o tamanho da amostra cresce. Além disso, em todos os cenários, vale observar que a penalização no critério BIC em relação ao critério AIC é sempre superior.

Utilizando ainda esses critérios de seleção, comparamos qual abordagem de estimação (bases de Fourier ou ondaletas de Haar) fornece menores valores de AIC e BIC para os modelos ajustados. Assim, para cada amostra simulada, ajustamos os modelos de regressão tipo Cox (3.10) e (4.1) considerando apenas os tempos deformados. Para as bases de Fourier fixamos, $J \in\{1,2,3\}$, e para as ondaletas Haar adotamos, $J \in\{0,1,2\}$, totalizando seis modelos ajustados. Dessa forma, consideramos como melhor abordagem (Fourier ou ondaletas de Haar) para estimar o efeito da covariável ao longo do tempo, o modelo que produzir o 
Tabela 5.5: Percentual do valor ótimo de J selecionado pelos critérios AIC e BIC considerando as ondaletas clássicas/deformadas, respectivamente e as bases de Fourier e tempos deformados.

\begin{tabular}{|c|c|c|c|c|c|c|c|c|}
\hline Base & Censura & Critério & $J$ & 400 & 600 & 800 & 1000 & 5000 \\
\hline \multirow{12}{*}{$\frac{\frac{\tilde{J}}{0}}{\frac{\pi}{0}}$} & \multirow{6}{*}{$0 \%$} & \multirow{3}{*}{ AIC } & 0 & $60 / 53$ & $67 / 58$ & $57 / 41$ & $50 / 30$ & $3 / 0$ \\
\hline & & & 1 & $30 / 33$ & $20 / 32$ & $34 / 42$ & $39 / 48$ & $50 / 25$ \\
\hline & & & 2 & $10 / 14$ & $13 / 10$ & $9 / 17$ & $11 / 22$ & $47 / 75$ \\
\hline & & \multirow{3}{*}{ BIC } & 0 & $98 / 96$ & $97 / 97$ & $98 / 98$ & $97 / 95$ & $70 / 17$ \\
\hline & & & 1 & $2 / 4$ & $3 / 3$ & $2 / 2$ & $3 / 5$ & $30 / 83$ \\
\hline & & & 2 & $0 / 0$ & $0 / 0$ & $0 / 0$ & $0 / 0$ & $0 / 0$ \\
\hline & \multirow{6}{*}{$30 \%$} & \multirow{4}{*}{ AIC } & 0 & $68 / 75$ & $70 / 70$ & $61 / 64$ & $49 / 56$ & $6 / 1$ \\
\hline & & & 1 & $24 / 19$ & $20 / 22$ & $27 / 28$ & $41 / 39$ & $59 / 55$ \\
\hline & & & 2 & $8 / 6$ & $10 / 8$ & $12 / 8$ & $10 / 5$ & $35 / 44$ \\
\hline & & & 0 & $98 / 100$ & $97 / 98$ & 99 / 98 & $97 / 98$ & $72 / 61$ \\
\hline & & \multirow[t]{2}{*}{ BIC } & 1 & $2 / 0$ & $3 / 2$ & $1 / 2$ & $3 / 2$ & $28 / 39$ \\
\hline & & & 2 & $0 / 0$ & $0 / 0$ & $0 / 0$ & $0 / 0$ & $0 / 0$ \\
\hline \multirow{12}{*}{ 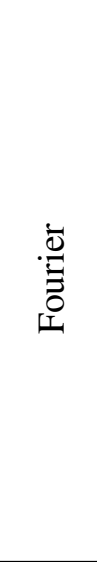 } & \multirow{5}{*}{$0 \%$} & \multirow{4}{*}{ AIC } & 1 & 56 & 56 & 55 & 29 & 0 \\
\hline & & & 2 & 21 & 28 & 32 & 48 & 15 \\
\hline & & & 3 & 23 & 16 & 13 & 23 & 85 \\
\hline & & & 1 & 98 & 98 & 99 & 96 & 31 \\
\hline & & \multirow[t]{2}{*}{ BIC } & 2 & 2 & 2 & 1 & 4 & 58 \\
\hline & \multirow{7}{*}{$30 \%$} & & 3 & 0 & 0 & 0 & 0 & 11 \\
\hline & & \multirow{4}{*}{ AIC } & 1 & 72 & 64 & 68 & 54 & 2 \\
\hline & & & 2 & 19 & 19 & 20 & 23 & 35 \\
\hline & & & 3 & 9 & 17 & 12 & 23 & 63 \\
\hline & & & 1 & 98 & 99 & 98 & 98 & 0 \\
\hline & & \multirow[t]{2}{*}{ BIC } & 2 & 2 & 1 & 2 & 2 & 72 \\
\hline & & & 3 & 0 & 0 & 0 & 0 & 28 \\
\hline
\end{tabular}

menor valor de AIC e BIC.

Apresentamos na Tabela 5.7 o percentual em que cada abordagem foi selecionada pelos critérios AIC e BIC produzido pelas 100 réplicas. Os resultados indicam ampla vantagem das bases de ondaletas de Haar em relação às séries de Fourier na estimação de $\beta_{0}(t)$, independente do tamanho amostral e nível de censura na amostra. Destacamos ainda maior preferência das ondaletas quando o critério BIC é utilizado.

A partir do valor ótimo de $J$ selecionado pelos critérios AIC e BIC, obtemos para cada cenário e procedimento de estimação as REQM produzidas pelos modelos ajustados. Na Tabela 5.8, apresentamos a média amostral e o desvio padrão das REQM quando os critérios de informação são utilizados para selecionar o nível mais apropriado de $J$.

É possível observar que a média amostral e o desvio padrão das REQM diminuem à medida que o tamanho da amostra aumenta. Na presença de censura, maiores médias e desvios padrão são observados em relação a dados completos, como esperado. Em todos os cenários, utilizar expansão em séries de Fourier acarreta maiores REQM. É importante ainda ressaltar que como o critério BIC seleciona frequentemente, para $\beta_{0}(t)=t^{1 / 2}$, o menor valor de $J$, menores REQM para esse critério são obtidas, no entanto adotar o critério AIC pode ser uma vantagem, uma vez que o nível selecionado pode representar com maior detalhe a função estimada.

Para dados completos, o critério BIC fornece menores médias para as REQM quando a estimação é realizada via ondaletas clássicas, enquanto para amostras censuradas as ondaletas deformadas produzem as menores REQM. Entre os dois critérios de seleção, em geral, o BIC fornece menores REQM para 
Tabela 5.6: Valores médios e (desvios padrão) das 100 repetições de AIC e BIC considerando os tempos deformados.

\begin{tabular}{|c|c|c|c|c|c|c|c|c|}
\hline Base & Censura & Critério & $J$ & 400 & 600 & 800 & 1000 & 5000 \\
\hline \multirow{12}{*}{$\begin{array}{l}\frac{\pi}{0} \\
\frac{\pi}{\pi} \\
\tilde{0}\end{array}$} & \multirow{6}{*}{$0 \%$} & \multirow{4}{*}{ AIC } & 0 & $3957(11,73)$ & $6416(12,82)$ & $9011(17,02)$ & $11710(17,37)$ & $74598(39,94)$ \\
\hline & & & 1 & $3958(11,50)$ & $6417(12,63)$ & $9012(16,96)$ & $11708(17,52)$ & $74580(40,35)$ \\
\hline & & & 2 & $3959(11,53)$ & $6418(12,40)$ & $9014(16,89)$ & $11709(17,26)$ & $74574(39,77)$ \\
\hline & & & 0 & $3970(11,73)$ & $6430(12,82)$ & $9027(17,02)$ & $11725(17,37)$ & $74618(39,94)$ \\
\hline & & \multirow[t]{2}{*}{ BIC } & 1 & $3978(11,50)$ & $6439(12,63)$ & $9036(16,96)$ & $11733(17,52)$ & $74612(40,35)$ \\
\hline & & & 2 & $3987(11,53)$ & $6449(12,40)$ & $9047(16,89)$ & $11743(17,26)$ & $74620(39,77)$ \\
\hline & \multirow{6}{*}{$30 \%$} & \multirow{4}{*}{ AIC } & 0 & $2850(88,38)$ & $4597(126,27)$ & $6478(123,88)$ & $8368(179,45)$ & $53281(494,14)$ \\
\hline & & & 1 & $2852(88,26)$ & $4598(126,22)$ & $6479(124,06)$ & $8369(179,90)$ & $53272(494,09)$ \\
\hline & & & 2 & $2853(88,44)$ & $4599(126,34)$ & $6480(124,09)$ & $8370(180,07)$ & $53269(494,47)$ \\
\hline & & & 0 & $2863(88,38)$ & $4610(126,27)$ & $6492(123,88)$ & $8384(179,45)$ & $53300(494,14)$ \\
\hline & & \multirow[t]{2}{*}{ BIC } & 1 & $2872(88,26)$ & $4620(126,22)$ & $6502(124,06)$ & $8394(179,90)$ & $53304(494,09)$ \\
\hline & & & 2 & $2881(88,44)$ & $4630(126,34)$ & $6513(124,09)$ & $8405(180,07)$ & $53315(494,47)$ \\
\hline \multirow{12}{*}{ 莺 } & \multirow{5}{*}{$0 \%$} & \multirow{4}{*}{ AIC } & 1 & $3955(10,72)$ & $6414(12,62)$ & $9011(16,50)$ & $11710(17,11)$ & $74592(37,53)$ \\
\hline & & & 2 & $3956(11,11)$ & $6415(12,52)$ & $9010(16,59)$ & $11707(17,47)$ & $74570(38,58)$ \\
\hline & & & 3 & $3959(11,68)$ & $6419(12,77)$ & $9013(16,73)$ & $11709(17,28)$ & $74566(38,68)$ \\
\hline & & & 1 & $3964(10,72)$ & $6424(12,62)$ & $9021(16,50)$ & $11719(17,11)$ & $74605(37,53)$ \\
\hline & & \multirow[t]{2}{*}{ BIC } & 2 & $3972(11,11)$ & $6433(12,52)$ & $9029(16,59)$ & $11727(17,47)$ & $74596(38,58)$ \\
\hline & \multirow{7}{*}{$30 \%$} & & 3 & $3991(11,68)$ & $6454(12,77)$ & $9051(16,73)$ & $11749(17,28)$ & $74618(38,68)$ \\
\hline & & \multirow{4}{*}{ AIC } & 1 & $2849(88,17)$ & $4595(126,32)$ & $6476(123,81)$ & $8366(179,70)$ & $53277(494,57)$ \\
\hline & & & 2 & $2851(88,38)$ & $4596(126,27)$ & $6477(123,95)$ & $8367(180,17)$ & $53266(494,19)$ \\
\hline & & & 3 & $2854(88,71)$ & $4600(125,85)$ & $6480(124,01)$ & $8371(180,30)$ & $53265(494,26)$ \\
\hline & & & 1 & $2857(88,17)$ & $4604(126,32)$ & $6485(123,81)$ & $8377(179,70)$ & $53290(494,57)$ \\
\hline & & \multirow[t]{2}{*}{ BIC } & 2 & $2867(88,38)$ & $4614(126,27)$ & $6495(123,95)$ & $8387(180,17)$ & $53292(494,19)$ \\
\hline & & & 3 & $2886(88,71)$ & $4635(125,85)$ & $6518(124,01)$ & $8410(180,30)$ & $53317(494,26)$ \\
\hline
\end{tabular}

Tabela 5.7: Percentual em que cada abordagem de estimação foi selecionado pelos critérios AIC e BIC considerando os tempos deformados.

\begin{tabular}{cccccc}
\hline & & \multicolumn{3}{c}{ AIC } & \multicolumn{2}{c}{ BIC } \\
\cline { 3 - 6 } Censura & $n$ & Ondaletas & Fourier & Ondaletas & Fourier \\
\hline \multirow{6}{*}{$0 \%$} & 400 & 78 & 22 & 97 & 3 \\
& 600 & 76 & 24 & 98 & 2 \\
& 800 & 79 & 21 & 94 & 6 \\
& 1000 & 71 & 29 & 88 & 12 \\
& 5000 & 91 & 9 & 96 & 4 \\
\cline { 2 - 6 } $30 \%$ & 400 & 75 & 25 & 97 & 3 \\
& 600 & 79 & 21 & 99 & 1 \\
& 800 & 80 & 20 & 96 & 4 \\
& 1000 & 79 & 21 & 97 & 3 \\
& 5000 & 83 & 17 & 98 & 2 \\
\hline
\end{tabular}

$n \leq 1000$, ao passo que em amostras maiores o critério AIC fornece as menores REQM. Como mencionado anteriormente, o critério BIC seleciona níveis mais baixos, em geral $J=0$ (ondaletas) e $J=1$ (Fourier). Levando em conta que esse comportamento também se aplica para outras formas do efeito da covariável, temos indicações em favor do uso do critério AIC em dados reais. 
Tabela 5.8: Média amostral e desvio padrão das REQM obtidas pelos critérios AIC e BIC para a seleção do valor ótimo J via ondaletas clássicas, deformadas e bases de Fourier, respectivamente.

\begin{tabular}{|c|c|c|c|c|}
\hline Censura & Critério & $n$ & Média & Desvio padrão \\
\hline \multirow{11}{*}{$0 \%$} & \multirow{5}{*}{ AIC } & 400 & $0,292 / 0,300 / 0,310$ & $0,064 / 0,076 / 0,056$ \\
\hline & & 600 & $0,231 / 0,238 / 0,251$ & $0,039 / 0,045 / 0,026$ \\
\hline & & 800 & $0,208 / 0,248 / 0,239$ & $0,024 / 0,060 / 0,026$ \\
\hline & & 1000 & $0,190 / 0,222 / 0,225$ & $0,021 / 0,030 / 0,019$ \\
\hline & & 5000 & $0,107 / 0,184 / 0,172$ & $0,004 / 0,015 / 0,007$ \\
\hline & \multirow{5}{*}{ BIC } & 400 & $0,234 / 0,232 / 0,263$ & $0,034 / 0,038 / 0,037$ \\
\hline & & 600 & $0,195 / 0,196 / 0,222$ & $0,018 / 0,020 / 0,016$ \\
\hline & & 800 & $0,190 / 0,194 / 0,222$ & $0,018 / 0,022 / 0,021$ \\
\hline & & 1000 & $0,176 / 0,184 / 0,208$ & $0,013 / 0,016 / 0,015$ \\
\hline & & 5000 & $0,139 / 0,160 / 0,177$ & $0,006 / 0,009 / 0,006$ \\
\hline & \multirow{5}{*}{ AIC } & 400 & $0,320 / 0,308 / 0,346$ & $0,074 / 0,078 / 0,087$ \\
\hline \multirow{9}{*}{$30 \%$} & & 600 & $0,268 / 0,271 / 0,287$ & $0,037 / 0,047 / 0,052$ \\
\hline & & 800 & $0,263 / 0,262 / 0,270$ & $0,035 / 0,038 / 0,046$ \\
\hline & & 1000 & $0,242 / 0,249 / 0,263$ & $0,029 / 0,032 / 0,036$ \\
\hline & & 5000 & $0,177 / 0,194 / 0,203$ & $0,013 / 0,012 / 0,014$ \\
\hline & \multirow{5}{*}{ BIC } & 400 & $0,300 / 0,280 / 0,306$ & $0,058 / 0,052 / 0,067$ \\
\hline & & 600 & $0,267 / 0,250 / 0,252$ & $0,028 / 0,035 / 0,042$ \\
\hline & & 800 & $0,260 / 0,243 / 0,256$ & $0,026 / 0,033 / 0,043$ \\
\hline & & 1000 & $0,260 / 0,236 / 0,240$ & $0,022 / 0,026 / 0,033$ \\
\hline & & 5000 & $0,224 / 0,206 / 0,208$ & $0,017 / 0,018 / 0,014$ \\
\hline
\end{tabular}




\section{Capítulo 6}

\section{Aplicações}

Neste capítulo, apresentamos a aplicação dos modelos propostos nos capítulos 4 e 5 a dois conjuntos de dados reais. Inicialmente reanalisaremos um conjunto de dados bem conhecido na literatura relativo a cirrose biliar primária em que as informações dos pacientes foram coletadas na Mayo Clinic. Já o segundo conjunto de dados refere-se ao tempo de vida de pacientes diagnosticados com qualquer tipo de câncer em estágio avançado, atendidos em dois hospitais públicos brasileiros especializados no tratamento de câncer.

\subsection{Dados de cirrose biliar primária}

O primeiro conjunto de dados analisado consiste em informações de cirrose biliar primária (PBC) coletadas na Mayo Clinic no período de janeiro de 1974 a maio de 1984 (Fleming e Harrington, 1991, apêndice. D). A cirrose biliar primária é uma doença crônica fatal do fígado e de causa desconhecida, com uma prevalência de 50 casos em cada um milhão.

A amostra é composta por 312 pacientes, com $60 \%$ de observações censuradas. O tempo de sobrevivência observado refere-se ao tempo em dias até a morte ou censura do paciente (média=2006, desvio padrão=1123,3) com o maior tempo observado de 4556 dias. A curva de Kaplan-Meier é apresentada na Figura 6.1. Dentre as informações coletadas podemos citar o gênero, idade, bilirrubina ( $\mathrm{mg} / \mathrm{dl})$, presença de edema e ascite, triglicérides $(\mathrm{mg} / \mathrm{dl})$, albumina $(\mathrm{mg} / \mathrm{dl})$ e tempo de protrombina em segundos.

Fleming e Harrington (1991) ajustaram o modelo de riscos proporcionais de Cox aos dados e eles identificaram como importantes variáveis preditoras a idade, indicador de edema, $\log$ (protrombina), $\log$ (albumina) e $\log$ (bilirrubina). Além disso, com o auxílio dos gráficos de resíduos padronizados de Schoenfeld concluise que as variáveis edema e $\log$ (protrombina) não satisfazem a suposição de riscos proporcionais.

O uso dos resíduos padronizados de Schoenfeld para avaliar a suposição de riscos proporcionais é baseado em um resultado, apresentado em Grambsch e Therneau (1994). Se a suposição da proporcionalidade dos riscos é válida, o gráfico dos resíduos padronizados de Schoenfeld mais a estimativa do coeficiente da regressão do modelo de Cox versus uma função do tempo, $g(t)$, deve ser uma linha horizontal. Inclinação zero mostra evidências a favor da proporcionalidade dos riscos. A fim de auxiliar na detecção de um possível violação de riscos proporcionais, uma curva suavizada, com bandas de confiança é adicionada ao gráfico.

A fim de ilustrar nosso método, consideramos apenas a variável explicativa log (protrombina) (média= 2,369 , desvio padrão $=0,089$ ) para avaliar o efeito do tempo de protrombina na taxa de falha. A protrombina é um elemento proteico da coagulação sanguínea, denominada também de fator II, sintetizada no fígado. O tempo de protrombina normal é de 11 a 14,6 segundos. Quanto maior é o tempo, menor é a concentração de protrombina no sangue e maior o risco de óbito. 


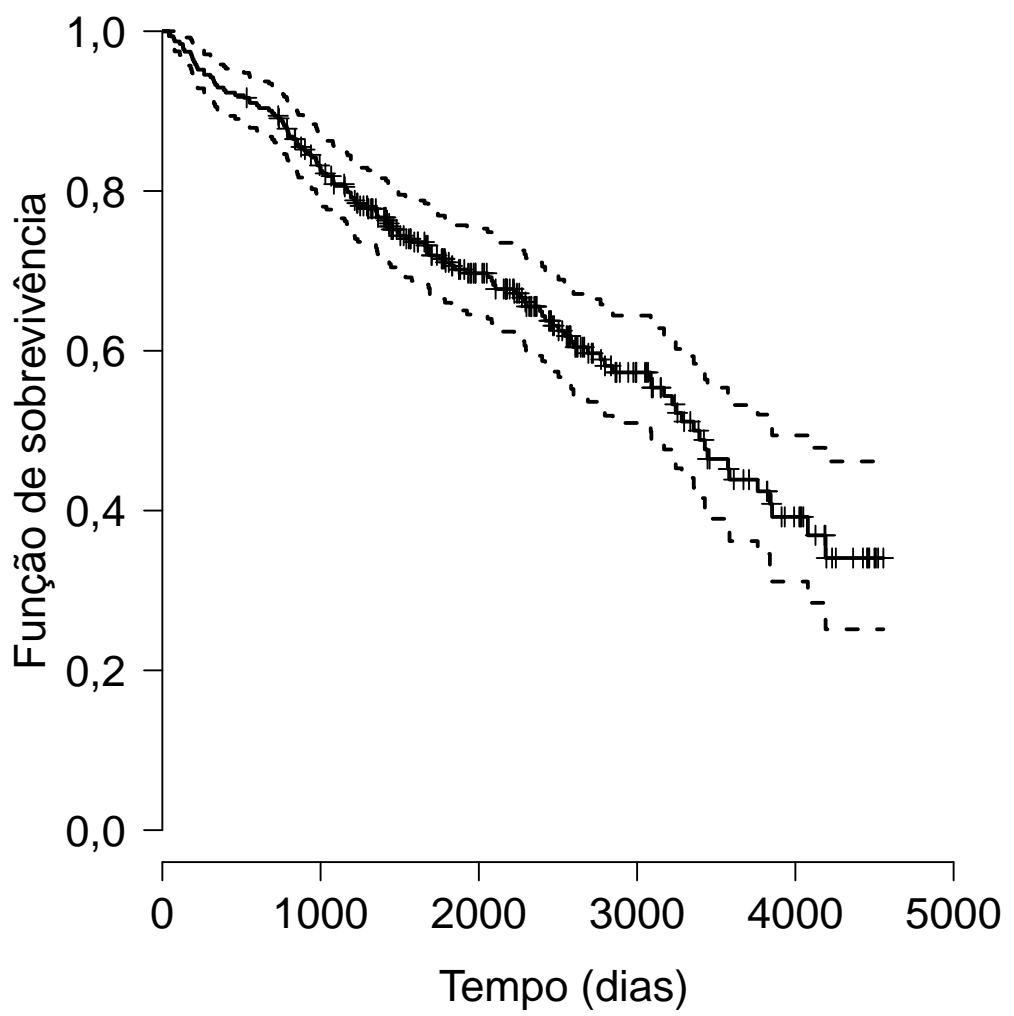

Figura 6.1: Curva de Kaplan-Meier e intervalo de confiança pontual de 95\%.

Inicialmente, apresentamos na Tabela 6.1 o ajuste do modelo de Cox considerando apenas essa covariável. De acordo com os resultados, quanto maior o tempo de protrombina, maior é o risco de morte do paciente.

Tabela 6.1: Resultados do ajuste do modelo de riscos proporcionais de Cox para os dados.

\begin{tabular}{cccc}
\hline Covariável & Coeficiente & Erro padrão & valor- $p$ \\
\hline $\log ($ protrombina $)$ & 5,171 & 0,690 & $<0,001$ \\
\hline
\end{tabular}

Para avaliar se a suposição de riscos proporcionais se sustenta, utilizamos o coeficiente de correlação de Pearson entre os resíduos padronizados de Schoenfeld e uma função do tempo $g(t)$, no caso $g(t)=t$, (Grambsch e Therneau, 1994), e também o método gráfico.

Na Tabela 6.2 é apresentado o resultado do teste de proporcionalidade dos riscos para o modelo de Cox, em que é possível observar fortes evidências de que a variável $\log$ (protrombina) tem um efeito variando ao longo do tempo, como pode ainda ser observado no gráfico de resíduos de Schoenfeld da Figura 6.2.

Tabela 6.2: Teste da suposição de riscos proporcionais com $g(t)=t$.

\begin{tabular}{cccc}
\hline Covariável & $\rho$ & $\chi^{2}$ & valor $-p$ \\
\hline $\log ($ protrombina $)$ & $-0,389$ & 9,130 & 0,003 \\
\hline
\end{tabular}

Como mencionamos anteriormente, a suposição de riscos proporcionais é violada e fazer o uso do modelo tradicional de Cox pode ser inadequado. Algumas soluções para contornar tal situação consistem em realizar estratificação ou redefinir a covariável, no entanto, neste trabalho, assumimos que o efeito da covariável varia ao longo do tempo, o que permite esclarecer o processo de maneira simples e apropriada. 


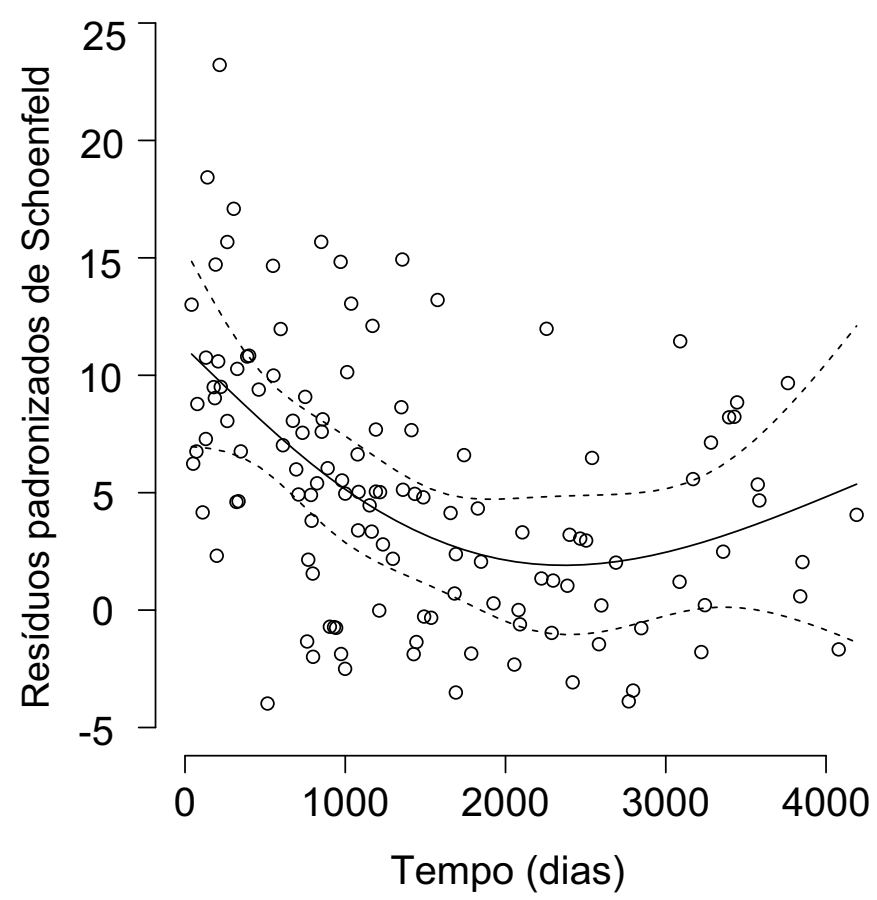

Figura 6.2: Estimativa de $\beta_{0}(t)$ baseado nos resíduos padronizados de Schoenfeld.

A fim de estimar o efeito da covariável sobre a taxa de falha, ajustamos os modelos de regressão tipo Cox em que o coeficiente dependente do tempo é representado em bases de Fourier (3.10) e ondaletas de Haar (4.1). Nos dois métodos de estimação estudados nos Capítulos 3 e 4 fixamos $J \in\{1,2,3\}$ (bases de Fourier) e $J \in\{0,1,2\}$ (bases de ondaletas de Haar). As estimativas dos parâmetros, erros padrão e o critério de seleção AIC para os modelos ajustados são apresentados nas Tabelas 6.3 e 6.4.

De acordo com o critério de seleção AIC, o modelo de regressão tipo Cox com o valor $J=1$ se destaca como o melhor modelo ajustado, independente do procedimento adotado. Vale destacar que as bases de Fourier produzem menores valores de AIC em relação às bases de ondaletas de Haar. Em geral, as ondaletas deformadas fornecem menores erros padrão quando comparados com as ondaletas clássicas.

Tabela 6.3: Séries de Fourier. Estimativa dos parâmetros (erros padrão) e o critério AIC.

\begin{tabular}{|c|c|c|c|c|c|c|c|c|c|}
\hline Fourier & $J$ & $c_{0}$ & $c_{1}$ & $c_{2}$ & $c_{3}$ & $d_{1}$ & $d_{2}$ & $d_{3}$ & AIC \\
\hline \multirow{3}{*}{ 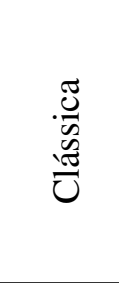 } & 1 & $\begin{array}{c}9,193 \\
(1,831)\end{array}$ & $\begin{array}{c}4,070 \\
(1,154)\end{array}$ & & & $\begin{array}{c}-0,045 \\
(1,233)\end{array}$ & & & 1231,78 \\
\hline & 2 & $\begin{array}{c}9,165 \\
(2,001)\end{array}$ & $\begin{array}{c}4,030 \\
(1,458)\end{array}$ & $\begin{array}{c}-0,006 \\
(1,106)\end{array}$ & & $\begin{array}{l}-0,056 \\
(1,328)\end{array}$ & $\begin{array}{c}0,089 \\
(1,488)\end{array}$ & & 1235,78 \\
\hline & 3 & $\begin{array}{c}7,254 \\
(2,495)\end{array}$ & $\begin{array}{c}3,035 \\
(1,760)\end{array}$ & $\begin{array}{c}-0,574 \\
(1,239)\end{array}$ & $\begin{array}{c}1,679 \\
(1,380)\end{array}$ & $\begin{array}{c}1,181 \\
(1,595)\end{array}$ & $\begin{array}{c}1,360 \\
(1,875)\end{array}$ & $\begin{array}{c}1,032 \\
(1,413)\end{array}$ & 1237,88 \\
\hline \multirow{3}{*}{ 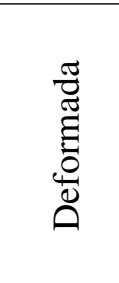 } & 1 & $\begin{array}{c}7,353 \\
(1,933)\end{array}$ & $\begin{array}{c}3,767 \\
(1,272)\end{array}$ & & & $\begin{array}{c}1,347 \\
(1,129)\end{array}$ & & & 1233,23 \\
\hline & 2 & $\begin{array}{c}7,570 \\
(1,941)\end{array}$ & $\begin{array}{c}3,491 \\
(1,403)\end{array}$ & $\begin{array}{c}1,126 \\
(1,142)\end{array}$ & & $\begin{array}{c}1,876 \\
(1,363)\end{array}$ & $\begin{array}{c}-0,750 \\
(1,306)\end{array}$ & & 1236,04 \\
\hline & 3 & $\begin{array}{c}7,564 \\
(1,986)\end{array}$ & $\begin{array}{c}3,546 \\
(1,474)\end{array}$ & $\begin{array}{c}0,376 \\
(1,472)\end{array}$ & $\begin{array}{c}1,366 \\
(1,182)\end{array}$ & $\begin{array}{c}1,755 \\
(1,299)\end{array}$ & $\begin{array}{c}-0,428 \\
(1,334)\end{array}$ & $\begin{array}{c}-0,010 \\
(1,331)\end{array}$ & 1238,71 \\
\hline
\end{tabular}


Tabela 6.4: Ondaletas Haar. Estimativa dos parâmetros (erros padrão) e o critério AIC.

\begin{tabular}{|c|c|c|c|c|c|c|c|c|c|c|}
\hline Ondaleta & $J$ & $c$ & $d_{0,0}$ & $d_{1,0}$ & $d_{1,1}$ & $d_{2,0}$ & $d_{2,1}$ & $d_{2,2}$ & $d_{2,3}$ & AIC \\
\hline \multirow{3}{*}{ 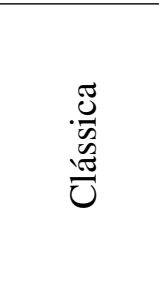 } & 0 & $\begin{array}{c}4,040 \\
(0,995)\end{array}$ & $\begin{array}{c}1,918 \\
(0,995)\end{array}$ & & & & & & & 1239,41 \\
\hline & 1 & $\begin{array}{c}3,972 \\
(1,383)\end{array}$ & $\begin{array}{c}1,299 \\
(1,383)\end{array}$ & $\begin{array}{c}1,348 \\
(0,636)\end{array}$ & $\begin{array}{c}-0,540 \\
(1,850)\end{array}$ & & & & & 1238,11 \\
\hline & 2 & $\begin{array}{c}4,512 \\
(1,462)\end{array}$ & $\begin{array}{c}0,476 \\
(1,462)\end{array}$ & $\begin{array}{c}1,313 \\
(0,704)\end{array}$ & $\begin{array}{l}-2,140 \\
(1,944)\end{array}$ & $\begin{array}{c}0,872 \\
(0,465)\end{array}$ & $\begin{array}{c}0,352 \\
(0,880)\end{array}$ & $\begin{array}{l}-1,758 \\
(1,093)\end{array}$ & $\begin{array}{c}-0,221 \\
(2,522)\end{array}$ & 1240,66 \\
\hline \multirow{3}{*}{$\begin{array}{l}\tilde{Z} \\
\tilde{\Xi} \\
\tilde{\Xi} \\
\tilde{0} \\
0\end{array}$} & 0 & $\begin{array}{c}4,100 \\
(0,923)\end{array}$ & $\begin{array}{c}2,198 \\
(0,923)\end{array}$ & & & & & & & 1237,01 \\
\hline & 1 & $\begin{array}{c}3,594 \\
(0,993)\end{array}$ & $\begin{array}{c}1,743 \\
(0,993)\end{array}$ & $\begin{array}{c}1,278 \\
(0,734)\end{array}$ & $\begin{array}{c}-0,782 \\
(1,197)\end{array}$ & & & & & 1236,93 \\
\hline & 2 & $\begin{array}{c}3,279 \\
(1,130)\end{array}$ & $\begin{array}{c}1,698 \\
(1,130)\end{array}$ & $\begin{array}{c}1,200 \\
(0,779)\end{array}$ & $\begin{array}{c}-1,062 \\
(1,395)\end{array}$ & $\begin{array}{c}0,887 \\
(0,477)\end{array}$ & $\begin{array}{c}0,532 \\
(0,994)\end{array}$ & $\begin{array}{c}0,172 \\
(1,364)\end{array}$ & $\begin{array}{c}0,078 \\
(1,424)\end{array}$ & 1240,23 \\
\hline
\end{tabular}

As estimativas do efeito da variável $\log$ (protrombina) ao longo do tempo obtidas via séries de Fourier e ondaletas Haar juntamente com os intervalos de confiança pontuais de 95\% são apresentados nas Figuras 6.3 a 6.6, respectivamente. Independente da abordagem de estimação, as curvas estimadas são consistentes com a estimativa não paramétrica obtida via resíduos de Schoenfeld (Figura 6.2). Além disso, observamos que as ondaletas deformadas produzem menores amplitudes para os intervalos de confiança pontuais em relação às ondaletas clássicas, como pode ser observado nos maiores tempos.

Examinamos também se a covariável $\log$ (protrombina) pode ser considerada como tendo efeito constante ao longo do tempo. O teste de hipótese de interesse é $H_{0}: c_{1}=\cdots=c_{J}=d_{1}=\cdots=d_{J}=0$ (bases de Fourier) e $H_{0}: d_{0,0}=\cdots=d_{J, 2^{J}-1}=0$ (bases de ondaletas de Haar) contra a hipótese alternativa de pelo menos um desses coeficientes ser diferente de 0 . Um resumo das estatísticas da razão de verossimilhanças (RV), escore e de Wald e os correspondentes valores- $p$ são apresentados na Tabela 6.5. Os resultados indicam que, em geral, há um forte efeito da variável $\log$ (protrombina) sobre a taxa de falha, mas que parece não ter significância após 2000 dias, se o problema é abordado via bases de ondaletas Haar (Figuras 6.5 e 6.6), enquanto por meio das séries de Fourier parece que o efeito é crescente após 2000 dias (Figuras 6.3 e $6.4)$.

Tabela 6.5: Testes da suposição de riscos proporcionais.

\begin{tabular}{|c|c|c|c|c|c|c|c|c|c|}
\hline \multirow[b]{2}{*}{ Base } & & \multirow{2}{*}{$J$} & \multirow{2}{*}{$\begin{array}{l}\text { Graus de } \\
\text { liberdade }\end{array}$} & \multicolumn{2}{|c|}{ RV } & \multicolumn{2}{|c|}{ Escore } & \multicolumn{2}{|c|}{ Wald } \\
\hline & & & & $X_{R V}^{2}$ & valor- $p$ & $X_{S C}^{2}$ & valor- $p$ & $X_{W}^{2}$ & valor- $p$ \\
\hline \multirow{6}{*}{ 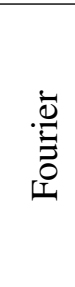 } & \multirow{3}{*}{ Clássica } & 1 & 2 & 14,849 & 0,001 & 13,566 & 0,001 & 12,715 & 0,002 \\
\hline & & 2 & 4 & 14,883 & 0,005 & 14,235 & 0,007 & 12,682 & 0,013 \\
\hline & & 3 & 6 & 20,556 & 0,002 & 16,042 & 0,014 & 14,962 & 0,021 \\
\hline & \multirow{3}{*}{ Deformada } & 1 & 2 & 16,935 & 0,001 & 11,189 & 0,004 & 10,642 & 0,005 \\
\hline & & 2 & 4 & 17,570 & 0,002 & 13,089 & 0,011 & 11,946 & 0,018 \\
\hline & & 3 & 6 & 18,913 & 0,004 & 15,058 & 0,002 & 13,643 & 0,034 \\
\hline \multirow{6}{*}{ 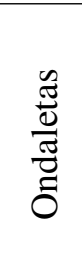 } & \multirow{3}{*}{ Clássica } & 0 & 1 & 7,031 & 0,008 & 3,967 & 0,046 & 3,722 & 0,054 \\
\hline & & 1 & 3 & 12,621 & 0,006 & 9,727 & 0,021 & 8,861 & 0,031 \\
\hline & & 2 & 7 & 16,175 & 0,024 & 18,192 & 0,011 & 14,250 & 0,047 \\
\hline & \multirow{3}{*}{ Deformada } & 0 & 1 & 9,181 & 0,002 & 6,154 & 0,013 & 5,676 & 0,017 \\
\hline & & 1 & 3 & 15,685 & 0,001 & 10,465 & 0,015 & 9,527 & 0,023 \\
\hline & & 2 & 7 & 22,284 & 0,002 & 14,734 & 0,039 & 14,250 & 0,017 \\
\hline
\end{tabular}


$J=1$

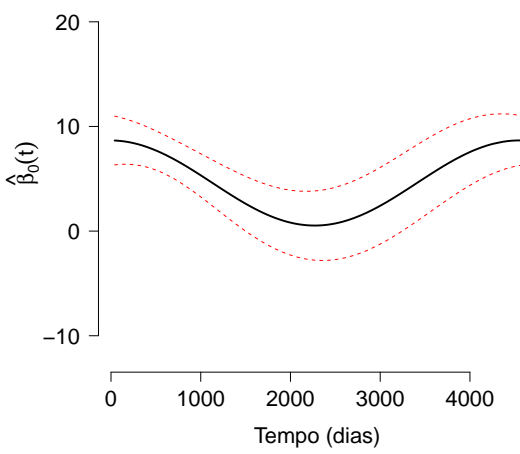

$\mathrm{J}=2$

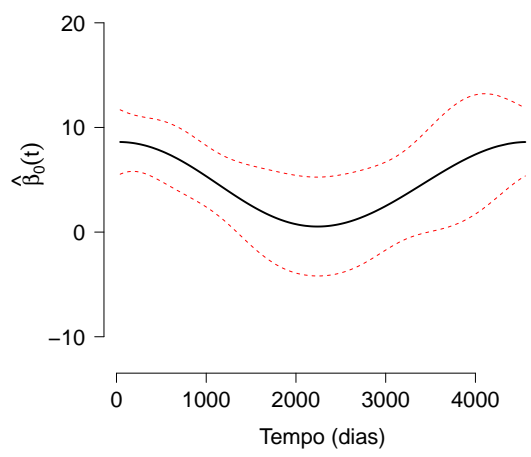

$J=3$

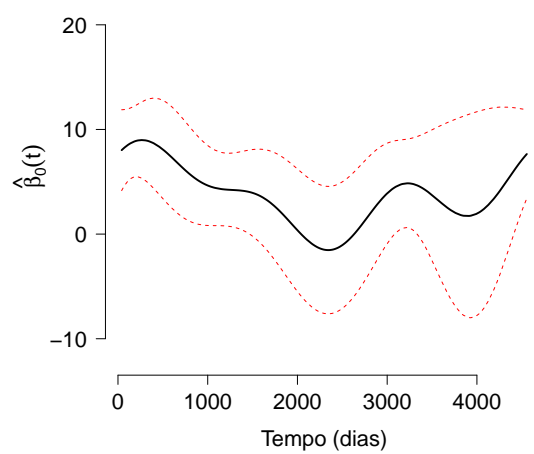

Figura 6.3: Séries de Fourier. Estimativa pontual (linha contínua) e intervalo de confiança pontual de $95 \%$ (linha tracejada) para o efeito da variável $\log$ (protrombina) ao longo do tempo para $J=1,2$ e 3 , respectivamente considerando os tempos clássicos.

$\mathrm{J}=1$

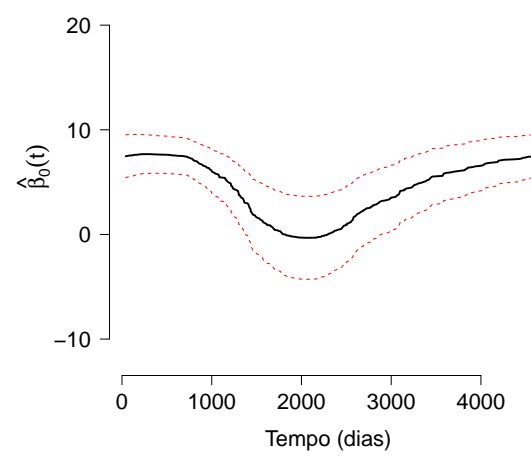

$\mathrm{J}=2$

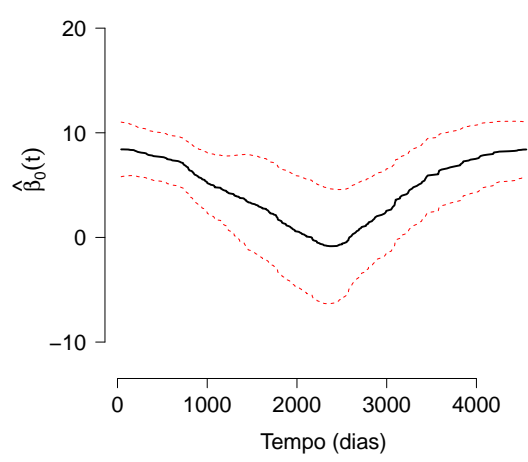

$\mathrm{J}=3$

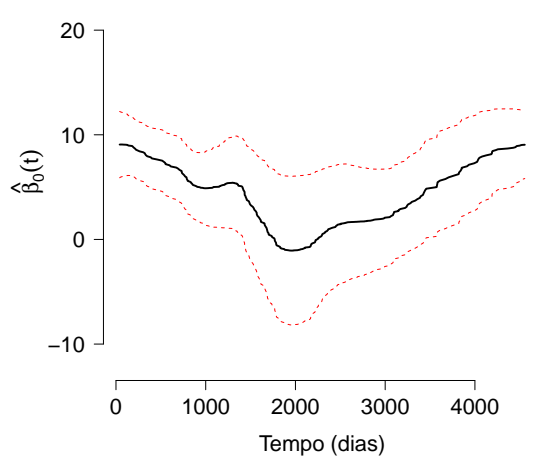

Figura 6.4: Séries de Fourier. Estimativa pontual (linha contínua) e intervalo de confiança pontual de $95 \%$ (linha tracejada) para o efeito da variável $\log$ (protrombina) ao longo do tempo para $J=1,2$ e 3 , respectivamente considerando os tempos deformados.
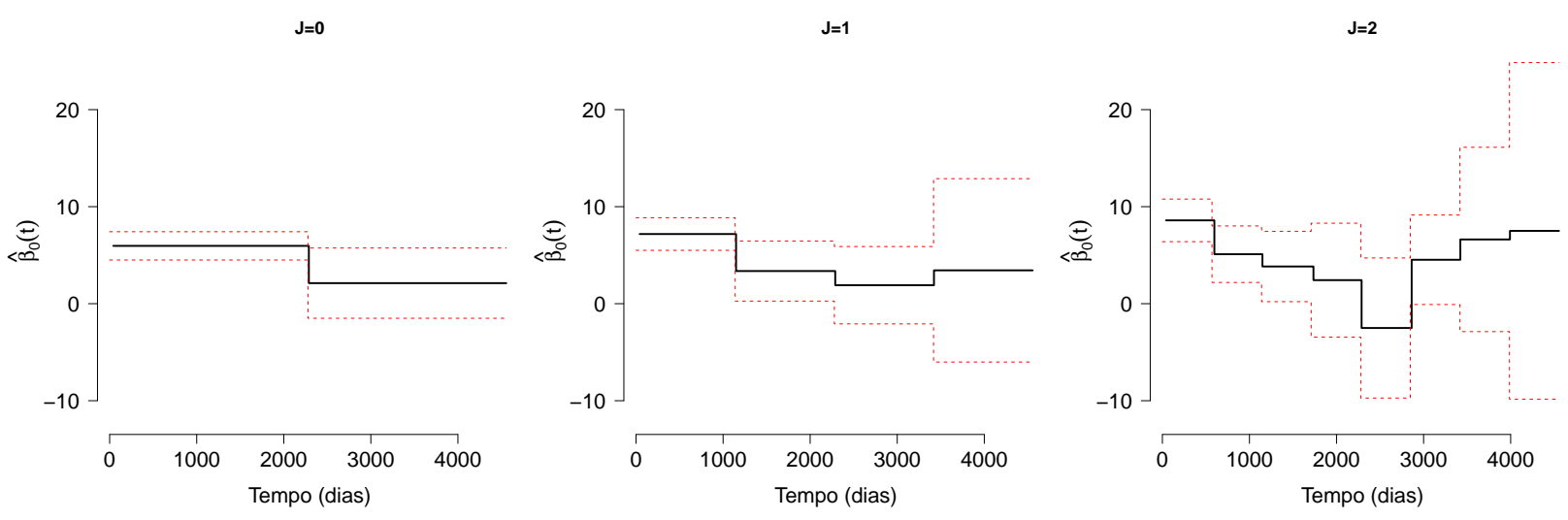

Figura 6.5: Ondaletas clássicas. Estimativa pontual (linha contínua) e intervalo de confiança pontual de $95 \%$ (linha tracejada) para o efeito da variável $\log$ (protrombina) ao longo do tempo para $J=0,1$ e 2 , respectivamente. 

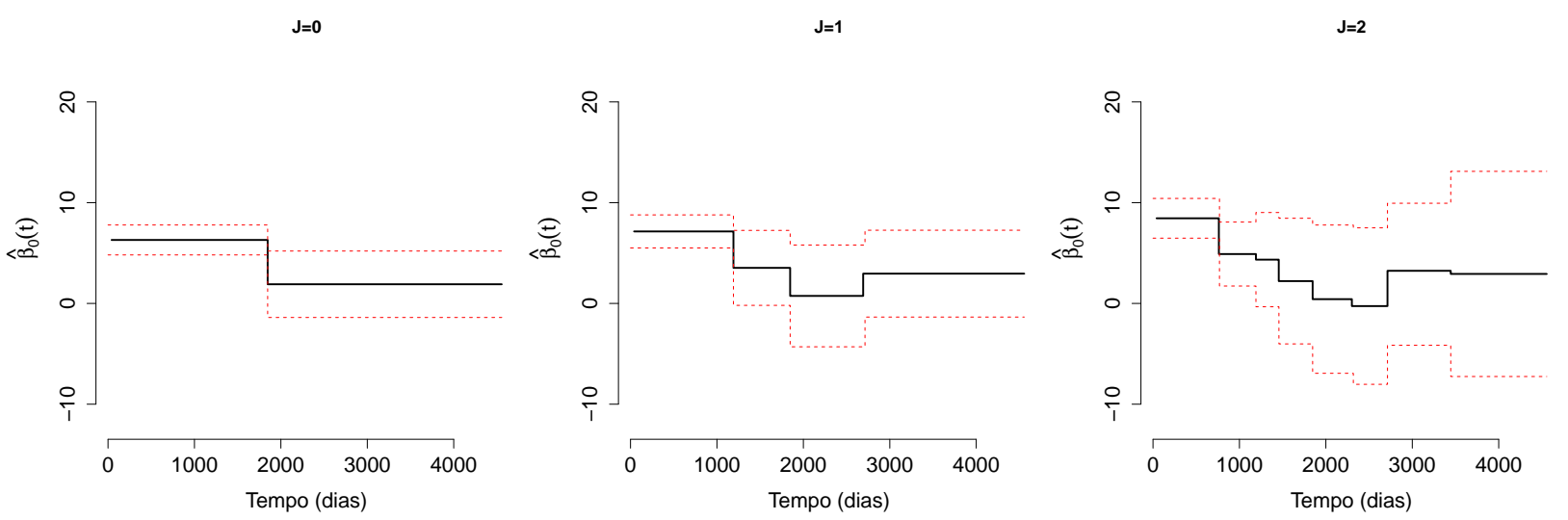

Figura 6.6: Ondaletas deformadas. Estimativa pontual (linha contínua) e intervalo de confiança pontual de 95\% (linha tracejada) para o efeito da variável $\log ($ protrombina) ao longo do tempo para $J=0,1$ e 2 , respectivamente.

Além do efeito da covariável ao longo do tempo, temos ainda o interesse em fazer predições da função de sobrevivência para um paciente específico, como mencionado nas Seções 3.3.3 e 4.1.3. Assim, para indivíduos com $\log$ (protrombina) $=2,361$ (mediana observada) e levando em conta os modelos selecionados pelo critério $\operatorname{AIC}(J=1)$, as curvas de sobrevivência estimadas via séries de Fourier e ondaletas de Haar são apresentadas, respectivamente, nas Figuras 6.7 e 6.8. Os intervalos de confiança pontuais de $95 \%$ são obtidos por meio dos percentis da reamostragem bootstrap, que é baseada em 1000 reamostras.

Os gráficos das Figuras 6.7 e 6.8 são bastante úteis. Por exemplo, para este particular paciente, um intervalo de confiança pontual para a probabilidade do paciente sobreviver mais que 3000 dias é $(0,531 ; 0,676)$ quando a estimação é obtida utilizando as ondaletas clássicas. Observe que o procedimento de predição é bem estável com respeito à escolha do método de transformação do tempo (clássico ou deformado) bem como a abordagem de estimação adotada. Por exemplo, para um paciente com $\log$ (protrombina) $=2,361$, a probabilidade de sobrevivência após 3000 dias varia de 0,534 a 0,671 para ondaletas deformadas, 0,530 a 0,668 utilizando as séries de Fourier e tempos clássicos e 0,535 a 0,670 quando a estimação é obtida por meio de séries de Fourier e tempos deformados, com $95 \%$ de confiança.

É importante mencionar que independente do procedimento de transformação dos tempos observados para o intervalo $[0,1)$, a estimativa pontual do efeito da covariável ao longo do tempo, assim como as curvas de sobrevivência, podem ser obtidas facilmente na escala de tempo original, o que é muito interessante na prática. No cenário em que os tempos são transformados de maneira clássica, a escala original de tempos é facilmente obtida multiplicando todos os tempos transformados pelo maior tempo observado da escala original. Por outro lado, se o método deformado é utilizado, a escala de tempo original é obtida aplicando a função inversa da distribuição empírica aos tempos transformados. 

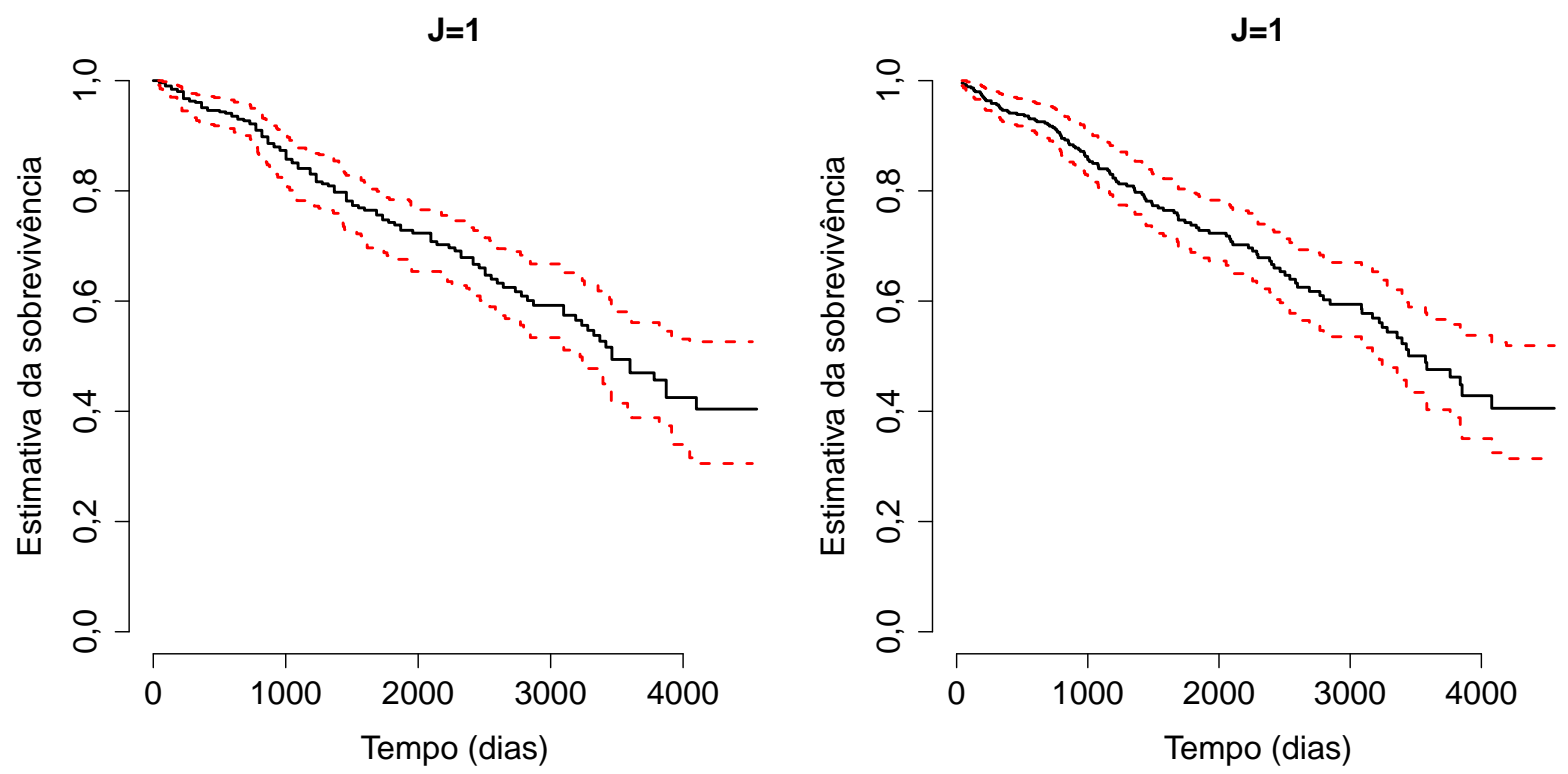

Figura 6.7: Séries de Fourier. Estimativa pontual (linha contínua) e intervalo de confiança pontual de 95\% (linha tracejada) obtido via bootstrap da função de sobrevivência para um paciente com $\log ($ protrombina $)=2,361$ estimado utilizando os tempos clássicos e deformados, respectivamente.
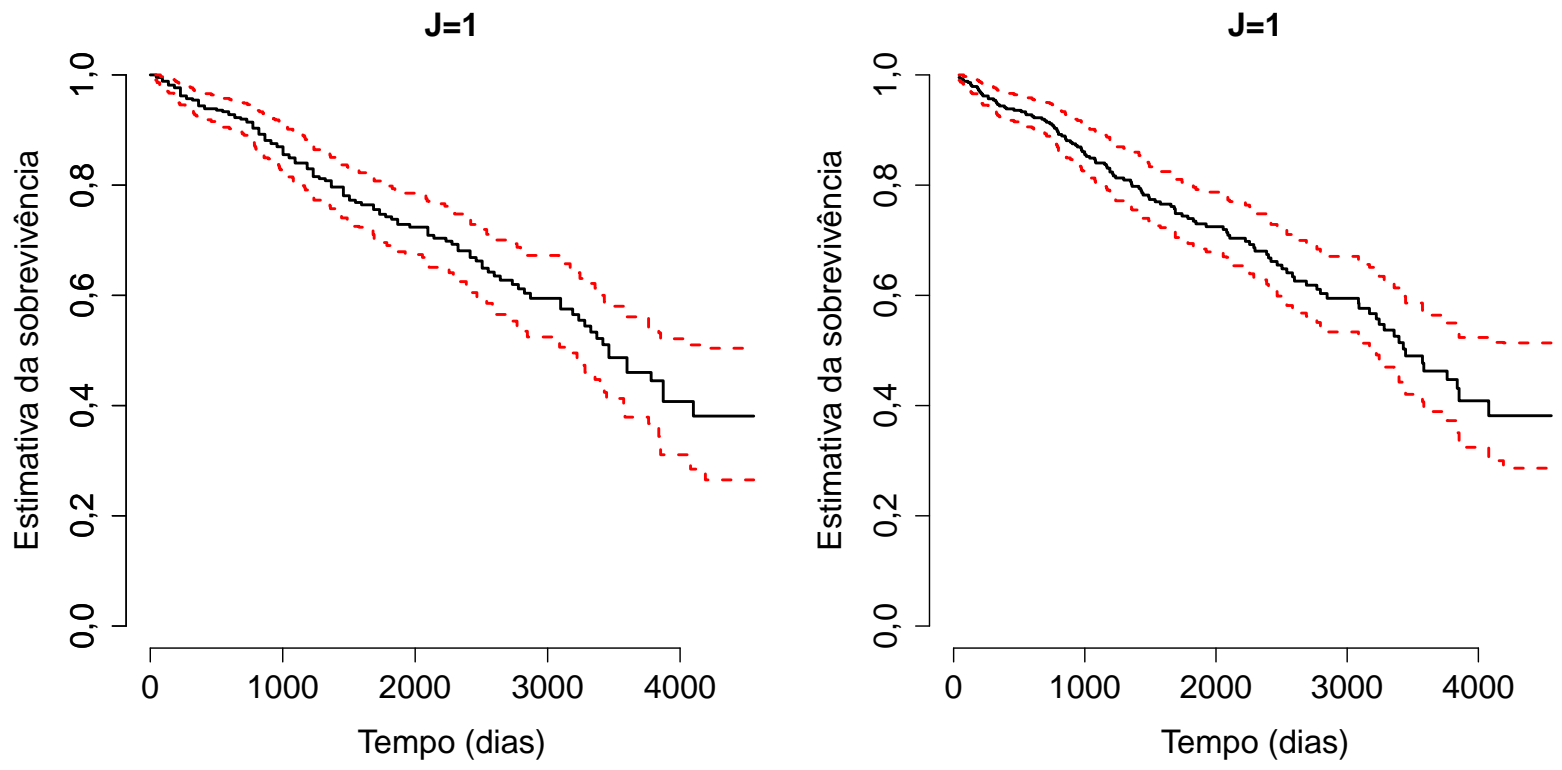

Figura 6.8: Ondaletas Haar. Estimativa pontual (linha contínua) e intervalo de confiança pontual de $95 \%$ (linha tracejada) obtido via bootstrap da função de sobrevivência para um paciente com $\log$ (protrombina) $=2,361$ estimado utilizando as ondaletas clássicas e deformadas, respectivamente. 


\subsection{Dados de câncer em estágio avançado}

O segundo conjunto de dados analisado refere-se ao tempo de sobrevida de pacientes diagnosticados com qualquer tipo de câncer em estágio avançado. $\mathrm{O}$ estudo foi realizado nas unidades de terapia intensiva (UTI) de dois hospitais públicos brasileiros especializados no tratamento de câncer: Instituto do Câncer do Estado de São Paulo (ICESP) e Hospital de Câncer de Barretos, no período de abril de 2009 a agosto de 2011. A amostra é composta por 773 pacientes, com $35 \%$ de observações censuradas. O tempo de vida observado baseia-se no tempo, em dias, até a morte ou censura do paciente desde a admissão na UTI (média $=334$, desvio padrão=316) com o maior tempo observado de 783 dias. Para cada paciente, foram registrados, o gênero, a idade, a concentração de bilirrubina total $(\mathrm{mg} / \mathrm{dl})$, níveis de ureia $(\mathrm{mg} / \mathrm{dl})$, sódio $(\mathrm{mg} / \mathrm{dl})$ e creatinina $(\mathrm{mg} / \mathrm{dl})$, indicador de cirrose, indicador de radioterapia, indicador de quimioterapia, bem como se realizou ou não cirurgia com o intuito de ser curativo ${ }^{1} / \operatorname{adjuvante~}^{2}$ ou paliativa ${ }^{3}$, além de outras variáveis.

A fim de ilustrar o nosso método, consideramos apenas a variável indicadora de cirurgia ( 0 : Não= 331; 1: Sim = 442) em que o objetivo principal é avaliar o efeito dessa covariável sobre a taxa de falha. Inicialmente, apresentamos na Figura 6.9 a curva de Kaplan-Meier estratificada pela covariável cirurgia, em que é possível observar maior probabilidade de sobrevivência para pacientes que foram submetidos a algum procedimento cirúrgico.

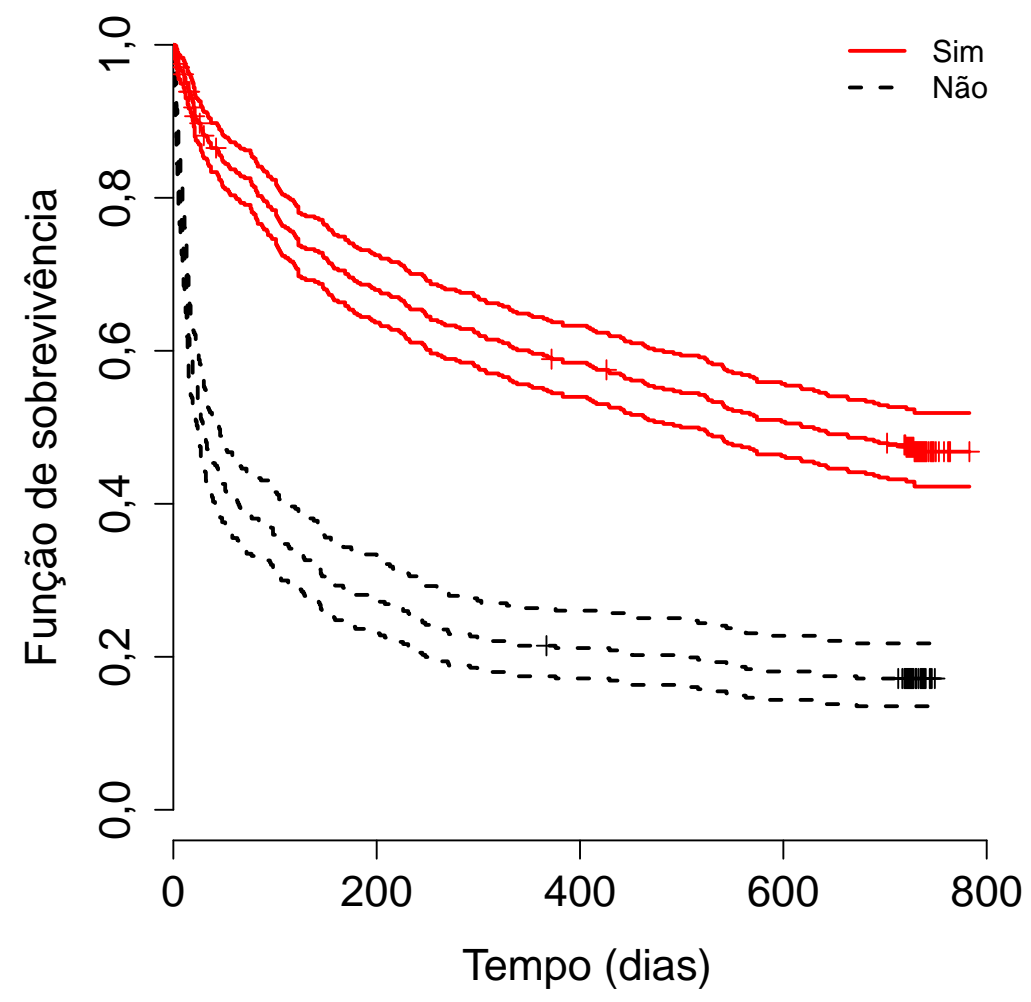

Figura 6.9: Curva de Kaplan-Meier estratificada pela covariável cirurgia juntamente com o intervalo de confiança pontual de $95 \%$.

\footnotetext{
${ }^{1}$ É o tipo mais comum de cirurgia de câncer em que o tumor cancerígeno e o tecido ao seu redor são totalmente removidos.

${ }^{2}$ Realizado após a cirurgia, o objetivo é destruir as células cancerígenas possivelmente remanescentes.

${ }^{3}$ Tem a finalidade de aliviar os efeitos colaterais causados por um tumor, melhorando a qualidade de vida do paciente oncológico.
} 
O resultado do ajuste do modelo de riscos proporcionais de Cox aos dados é apresentado na Tabela 6.6. De acordo com o resultado, pacientes que passaram por cirurgia apresentam menor risco de morte, como esperado.

Tabela 6.6: Resultados do ajuste do modelo de riscos proporcionais de Cox para os dados.

\begin{tabular}{ccccc}
\hline Covariável & Coeficiente & Risco relativo & Erro padrão & valor- $p$ \\
\hline Indicador de cirurgia & $-1,072$ & 0,342 & 0,091 & $<0,001$ \\
\hline
\end{tabular}

Para avaliar a suposição de riscos proporcionais do modelo de Cox, utilizamos o coeficiente de correlação de Pearson entre os resíduos padronizados de Schoenfeld e uma função $g(t)=t$, e também o método gráfico.

Na Tabela 6.7 é apresentado o resultado do teste de proporcionalidade dos riscos para o modelo de Cox, em que é possível observar fortes evidências de que a cirurgia tem um efeito variante ao longo do tempo, como pode ser observado ainda por meio do gráfico de resíduos de Schoenfeld apresentado na Figura 6.10.

Tabela 6.7: Teste da suposição de riscos proporcionais com $g(t)=t$.

\begin{tabular}{cccc}
\hline Covariável & $\rho$ & $\chi^{2}$ & valor- $p$ \\
\hline Indicador de cirurgia & $-0,264$ & 31,8 & $<0,001$ \\
\hline
\end{tabular}

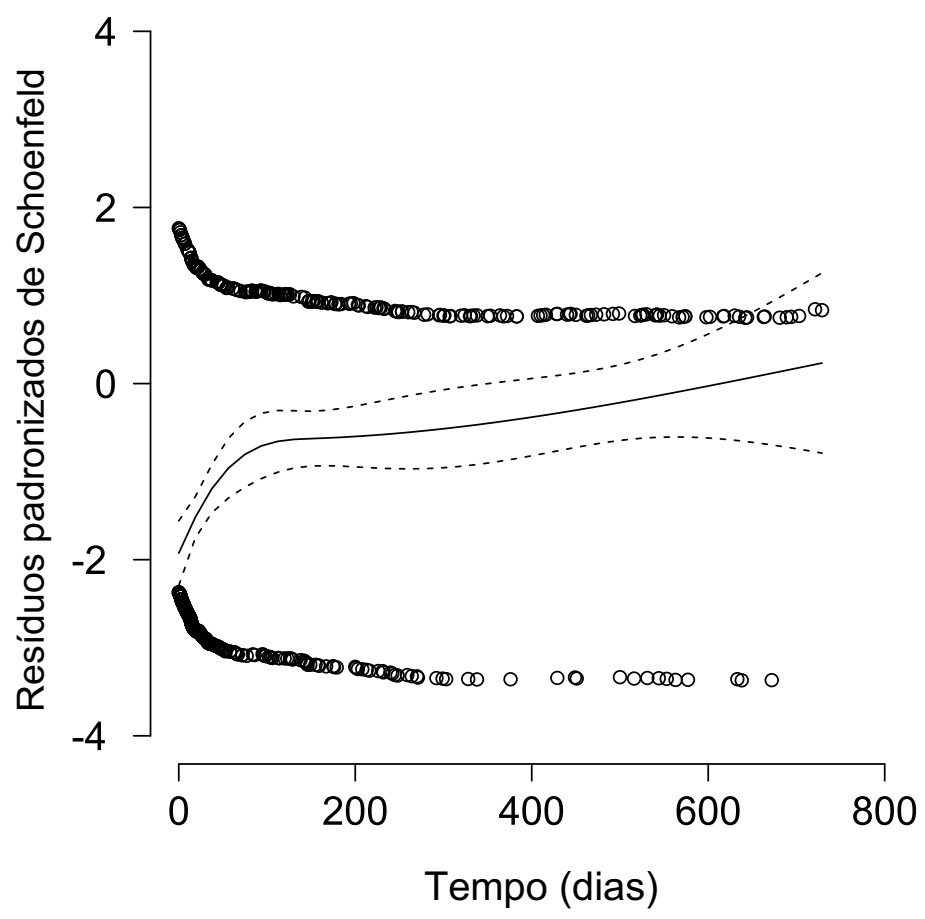

Figura 6.10: Estimativa de $\beta_{0}(t)$ basado nos resíduos padronizados de Schoenfeld.

Como observamos, a suposição de riscos proporcionais é violada e fazer o uso do modelo tradicional de Cox pode ser inadequado. Desta forma, a fim de esclarecer o processo de uma maneira mais apropriada, assumimos que o efeito da covariável varia ao longo do tempo. Além disso, temos também o interesse em conhecer e estimar o comportamento da função de risco basal na taxa de falha. Para isso, ajustamos aos dados os modelos de regressão tipo Cox via séries de Fourier (3.16) e ondaletas de Haar (4.9). Como 
mencionado, o número de coeficientes dos modelos aumentam à medida que o valor de $J$ cresce e, devido a essa razão, nas duas abordagens fixamos $J \in\{1,2\}$ (bases de Fourier) e $J \in\{0,1\}$ (bases de ondaletas Haar).

É importante mencionar que devido aos problemas obtidos na estimação dos parâmetros do modelo tipo Cox via séries de Fourier, o método de estimação alternativo em duas etapas foi utilizado. Por outro lado, no cenário em que as bases de ondaletas de Haar são consideradas, a estimação dos parâmetros em uma única etapa não apresentou problemas de otimização, e assim, esse método foi considerado.

As estimativas dos parâmetros, erros padrão e o critério AIC para os modelos ajustados via bases de Fourier e ondaletas de Haar são apresentadas, respectivamente, nas Tabelas 6.8 e 6.9. Vale ressaltar que no cenário em que o procedimento em duas etapas (bases de Fourier) é utilizado, o valor do critério de seleção AIC é obtido pela função de verossimilhança parcial (primeira etapa), ao passo que a estimação conjunta dos parâmetros (uma única etapa), o critério de informação AIC é calculado por meio da função de verossimilhança completa.

De acordo com o critério AIC, para as bases de Fourier, os menores valores de AIC são observados para $J=2$, independente do método de transformação dos tempos observados. Ao passo que para as bases de ondaletas de Haar, o modelo com nível de resolução $J=1$ se destaca como o melhor modelo ajustado, independente de ondaletas clássicas ou deformadas. Todavia, o menor valor de AIC, entre os modelos ajustados, ocorre para as ondaletas clássicas.

Tabela 6.8: Séries de Fourier. Estimativa dos parâmetros (erros padrão) e o critério AIC obtidos em duas etapas.

\begin{tabular}{|c|c|c|c|c|c|c|c|c|c|c|c|c|}
\hline & $J$ & $a_{0}$ & $a_{1}$ & $a_{2}$ & $b_{1}$ & $b_{2}$ & $c_{0}$ & $c_{1}$ & $c_{2}$ & $d_{1}$ & $d_{2}$ & AIC \\
\hline.$\frac{\pi}{\infty}$ & 1 & $\begin{array}{c}6,177 \\
(0,002)\end{array}$ & $\begin{array}{c}1,571 \\
(0,001)\end{array}$ & & $\begin{array}{c}2,505 \\
(0,001)\end{array}$ & & $\begin{array}{l}-1,250 \\
(0,294)\end{array}$ & $\begin{array}{l}-0,797 \\
(0,165)\end{array}$ & & $\begin{array}{l}-0,006 \\
(0,193)\end{array}$ & & 6068,30 \\
\hline$\frac{\sqrt[n]{0}}{0}$ & 2 & $\begin{array}{c}4,827 \\
(0,002)\end{array}$ & $\begin{array}{c}1,124 \\
(0,001)\end{array}$ & $\begin{array}{c}1,032 \\
(0,001)\end{array}$ & $\begin{array}{c}1,010 \\
(0,001)\end{array}$ & $\begin{array}{c}1,115 \\
(0,001)\end{array}$ & & & & $\begin{array}{l}-0,206 \\
(0,222)\end{array}$ & $\begin{array}{c}0,360 \\
(0,202)\end{array}$ & 6065,32 \\
\hline$\underset{\overparen{\Xi}}{\tilde{\Xi}}$ & 1 & $\begin{array}{c}7,728 \\
(0,002)\end{array}$ & $\begin{array}{l}-1,641 \\
(0,002)\end{array}$ & & $\begin{array}{c}1,613 \\
(0,001)\end{array}$ & & $\begin{array}{l}-2,151 \\
(0,286)\end{array}$ & $\begin{array}{l}-0,660 \\
(0,165)\end{array}$ & & $\begin{array}{l}-0,516 \\
(0,190)\end{array}$ & & 6051,82 \\
\hline อั & 2 & $\begin{array}{c}5,469 \\
(0,002)\end{array}$ & $\begin{array}{c}0,951 \\
(0,001)\end{array}$ & $\begin{array}{c}1,598 \\
(0,001)\end{array}$ & $\begin{array}{c}0,999 \\
(0,003)\end{array}$ & $\begin{array}{c}0,511 \\
(0,002)\end{array}$ & $\begin{array}{l}-1,411 \\
(0,511)\end{array}$ & $\begin{array}{c}-0,371 \\
(0,262)\end{array}$ & $\begin{array}{c}-0,334 \\
(0,192)\end{array}$ & $\begin{array}{l}-1,119 \\
(0,381)\end{array}$ & $\begin{array}{l}-0,397 \\
(0,259)\end{array}$ & 6051,56 \\
\hline
\end{tabular}

Tabela 6.9: Ondaletas Haar. Estimativa dos parâmetros (erros padrão) e o critério AIC obtidos em uma etapa.

\begin{tabular}{|c|c|c|c|c|c|c|c|c|c|c|}
\hline Ondaleta & $J$ & $a$ & $b_{0,0}$ & $b_{1,0}$ & $b_{1,1}$ & $c$ & $d_{0,0}$ & $d_{1,0}$ & $d_{1,1}$ & AIC \\
\hline \multirow{2}{*}{$\begin{array}{l}\tilde{U} \\
\tilde{U} \\
\tilde{U} \\
\tilde{U}\end{array}$} & 0 & $\begin{array}{c}2,727 \\
(0,168)\end{array}$ & $\begin{array}{c}2,240 \\
(0,168)\end{array}$ & & & $\begin{array}{c}-0,712 \\
(0,164)\end{array}$ & $\begin{array}{l}-0,731 \\
(0,164)\end{array}$ & & & 203,88 \\
\hline & 1 & $\begin{array}{c}2,312 \\
(0,146)\end{array}$ & $\begin{array}{c}1,861 \\
(0,146)\end{array}$ & $\begin{array}{c}2,106 \\
(0,186)\end{array}$ & $\begin{array}{c}0,115 \\
(0,090)\end{array}$ & $\begin{array}{l}-0,472 \\
(0,195)\end{array}$ & $\begin{array}{c}-0,602 \\
(0,195)\end{array}$ & $\begin{array}{l}-0,297 \\
(0,099)\end{array}$ & $\begin{array}{l}-0,177 \\
(0,257)\end{array}$ & 68,34 \\
\hline \multirow{2}{*}{ 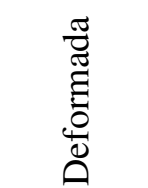 } & 0 & $\begin{array}{c}2,004 \\
(0,155)\end{array}$ & $\begin{array}{c}0,404 \\
(0,155)\end{array}$ & & & $\begin{array}{c}-0,764 \\
(0,112)\end{array}$ & $\begin{array}{c}-0,480 \\
(0,112)\end{array}$ & & & 623,52 \\
\hline & & $\begin{array}{c}1,801 \\
(0,130)\end{array}$ & $\begin{array}{c}0,700 \\
(0,130)\end{array}$ & $\begin{array}{c}-0,208 \\
(0,121)\end{array}$ & $\begin{array}{c}0,731 \\
(0,129)\end{array}$ & $\begin{array}{c}-0,322 \\
(0,784)\end{array}$ & $\begin{array}{l}-1,071 \\
(0,784)\end{array}$ & $\begin{array}{l}-0,340 \\
(0,083)\end{array}$ & $\begin{array}{c}-0,766 \\
(1,074)\end{array}$ & 524,38 \\
\hline
\end{tabular}

As estimativas do efeito da cirurgia ao longo do tempo obtidas via representação em bases de Fourier e ondaletas de Haar, juntamente com os intervalos de confiança pontuais de $95 \%$ são apresentados nas Figuras 6.11 a 6.14, respectivamente. Independente da abordagem, em geral as curvas estimadas para o efeito da covariável cirurgia são consistentes com a estimativa não paramétrica obtida por meio dos resíduos de Schoenfeld (Figura 6.10). No entanto, é importante mencionar que o procedimento de estimação via séries de Fourier e tempos deformados fornece estimativas instáveis para $t>700$, isso ocorre devido à alta 
frequência de observações censuradas e também às poucas falhas para $t>700$, como pode ser observado na Figura 6.9.

Avaliamos ainda se o efeito da covariável cirurgia sobre a taxa de falha pode ser considerado constante ao longo do tempo. O teste de hipótese de interesse é $H_{0}: c_{1}=\cdots=c_{J}=d_{1}=\cdots=d_{J}=0$ (bases de Fourier) e $H_{0}: d_{0,0}=\cdots=d_{J, 2^{J}-1}=0$ (bases de ondaletas de Haar) contra a hipótese alternativa de pelo menos um desses coeficientes ser diferente de 0 . Um resumo das estatísticas da razão de verossimilhanças (RV), escore e de Wald e os correspondentes valores- $p$ são apresentados na Tabela 6.10. Os resultados indicam que há um forte efeito da cirurgia sobre a taxa de falha, em especial, parece ser crescente ao longo do tempo, como pode ser visto principalmente quando as ondaletas de Haar são consideradas (Figuras 6.13 e 6.14).

Como é também de interesse, verificamos se o risco basal pode ser considerado constante ao longo do tempo. Neste caso, a hipótese de interesse é $H_{0}: a_{1}=\cdots=a_{J}=b_{1}=\cdots=b_{J}=0$ (bases de Fourier) e $H_{0}: b_{0,0}=\cdots=b_{J, 2^{J}-1}=0$ (bases de ondaletas de Haar) contra a hipótese alternativa de pelo menos um desses coeficientes ser diferente de 0 . Em todos os cenários encontramos indícios de risco basal variando ao longo do tempo, que parece diminuir ao longo do tempo.

Tabela 6.10: Testes de hipótese da suposição de riscos proporcionais.

\begin{tabular}{|c|c|c|c|c|c|c|c|c|c|}
\hline & \multirow{2}{*}{$J$} & \multirow{2}{*}{$\begin{array}{l}\text { Graus de } \\
\text { liberdade }\end{array}$} & \multicolumn{2}{|c|}{ RV } & \multicolumn{2}{|c|}{ Escore } & \multicolumn{2}{|c|}{ Wald } \\
\hline & & & & $X_{R V}^{2}$ & valor- $p$ & $X_{S C}^{2}$ & valor- $p$ & $X_{W}^{2}$ & valor- $p$ \\
\hline \multirow{4}{*}{ 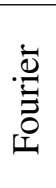 } & \multirow{2}{*}{ Clássica } & 1 & 2 & 24,724 & $<0,001$ & 24,489 & $<0,001$ & 23,484 & $<0,001$ \\
\hline & & 2 & 4 & 33,402 & $<0,001$ & 30,886 & $<0,001$ & 29,361 & $<0,001$ \\
\hline & \multirow{2}{*}{ Deformada } & 1 & 2 & 42,906 & $<0,001$ & 40,289 & $<0,001$ & 38,499 & $<0,001$ \\
\hline & & 2 & 4 & 47,165 & $<0,001$ & 42,969 & $<0,001$ & 40,225 & $<0,001$ \\
\hline \multirow{4}{*}{ 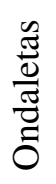 } & \multirow{2}{*}{ Clássica } & 0 & 1 & 23,988 & $<0,001$ & 22,689 & $<0,001$ & 19,968 & $<0,001$ \\
\hline & & 1 & 3 & 31,263 & $<0,001$ & 21,227 & $<0,001$ & 26,920 & $<0,001$ \\
\hline & \multirow{2}{*}{ Deformada } & 0 & 1 & 19,764 & $<0,001$ & 19,022 & $<0,001$ & 18,384 & $<0,001$ \\
\hline & & 1 & 3 & 40,158 & $<0,001$ & 8,651 & 0,034 & 33,117 & $<0,001$ \\
\hline
\end{tabular}

Utilizamos ainda os dados para ilustrar os métodos de predição da função de sobrevivência para a covariável cirurgia, como mencionado nas Seções 3.4.3 e 4.2.3. Considerando apenas os modelos selecionados via o critério $\operatorname{AIC}(J=2$ Fourier e $J=1$ ondaletas), apresentamos nas Figuras 6.15 e 6.16, respectivamente, as curvas de sobrevivência estimadas via bases de Fourier e ondaletas Haar, além dos correspondentes intervalos de confiança pontuais de $95 \%$ obtidos por meio da técnica de reamostragem bootstrap baseada em 1000 reamostras.

Os gráficos apresentados nas Figuras 6.15 e 6.16 são bem úteis. Por exemplo, se considerarmos as bases de ondaletas clássicas, a probabilidade de um paciente que passou por cirúrgica sobreviver mais de 600 dias varia de 0,532 a 0,448 , enquanto os que não passaram por cirurgia varia de 0,080 a 0,143 . Destacamos ainda se as ondaletas deformadas são adotadas, a probabilidade varia de 0,502 a 0,575) para os pacientes que passaram por cirurgia, por outro lado, os pacientes que não passaram por cirurgia varia de 0,155 a 0,241 .

No cenário em que as bases de Fourier são utilizadas, os intervalos de confiança obtidos via transformações clássicas e deformadas são, respectivamente, $\operatorname{Sim}=(0,455 ; 0,548)$, Não $=(0,148 ; 0,224)$ e $\operatorname{Sim}=(0,448 ; 0,548)$, Não $=(0,154 ; 0,220)$. Observe que o procedimento de predição é bem estável com respeito à escolha do método de transformação dos tempos observados (clássico ou deformado) bem como a base adotada. Além disso, as curvas estimadas, assim como os intervalos de confiança (bootstrap) estão bem próximas das curvas de Kaplan-Meier (Figura 6.9). 

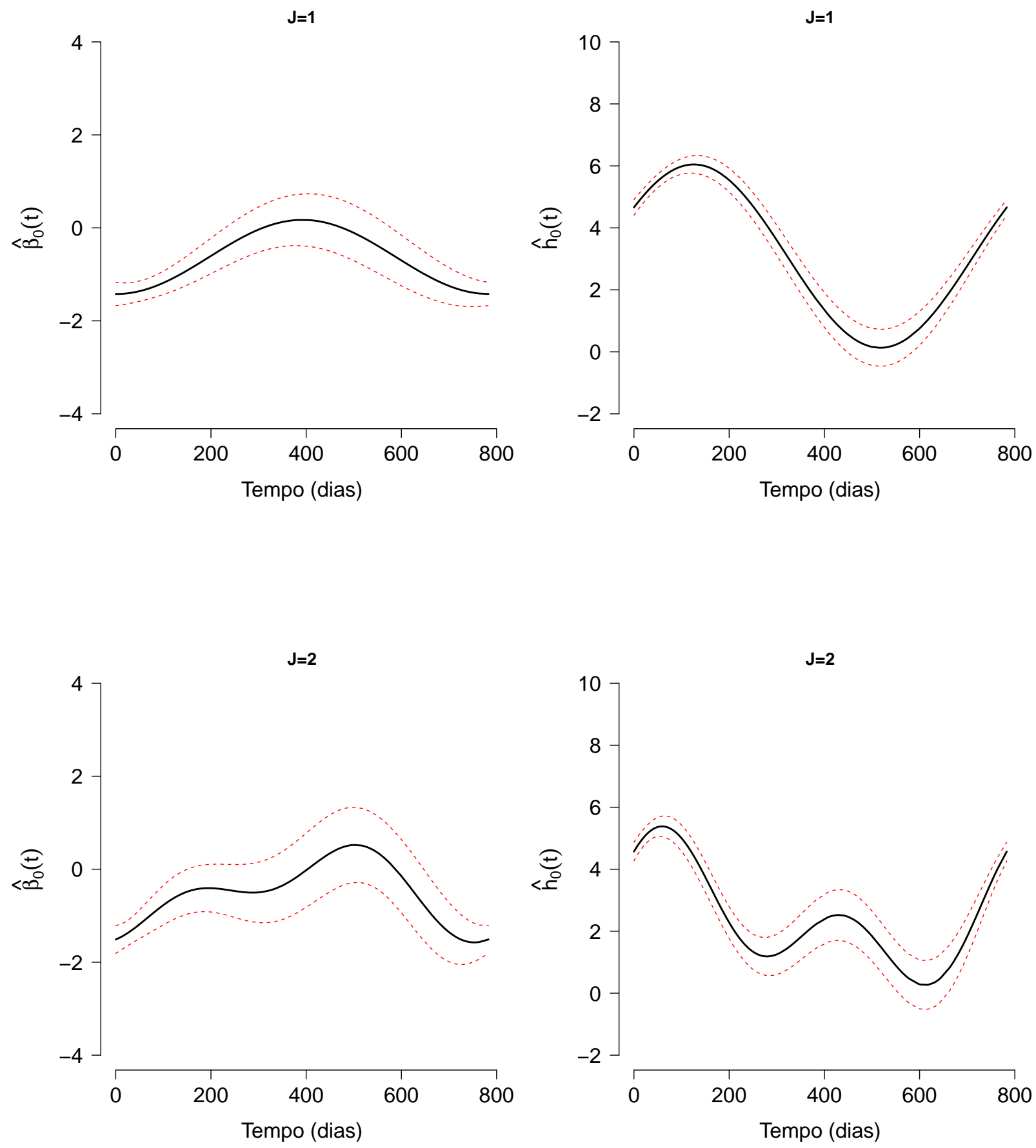

Figura 6.11: Séries de Fourier via transformação clássica. Estimativa pontual (linha contínua) e intervalo de confiança pontual de $95 \%$ (linha tracejada) para o efeito da cirurgia ao longo do tempo e função de risco basal, respectivamente. 

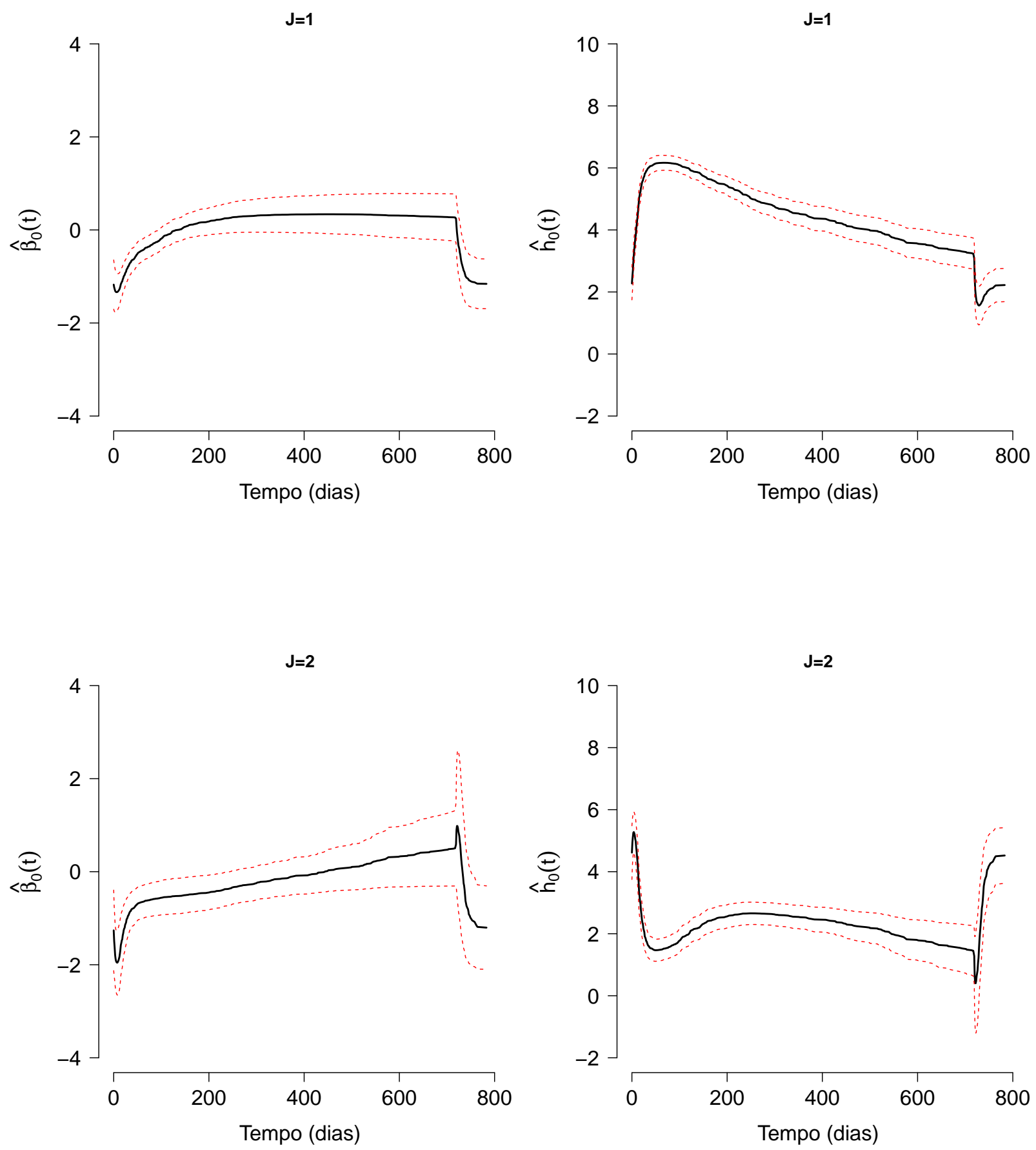

Figura 6.12: Séries de Fourier via transformação deformada. Estimativa pontual (linha contínua) e intervalo de confiança pontual de $95 \%$ (linha tracejada) para o efeito da cirurgia ao longo do tempo e função de risco basal, respectivamente. 

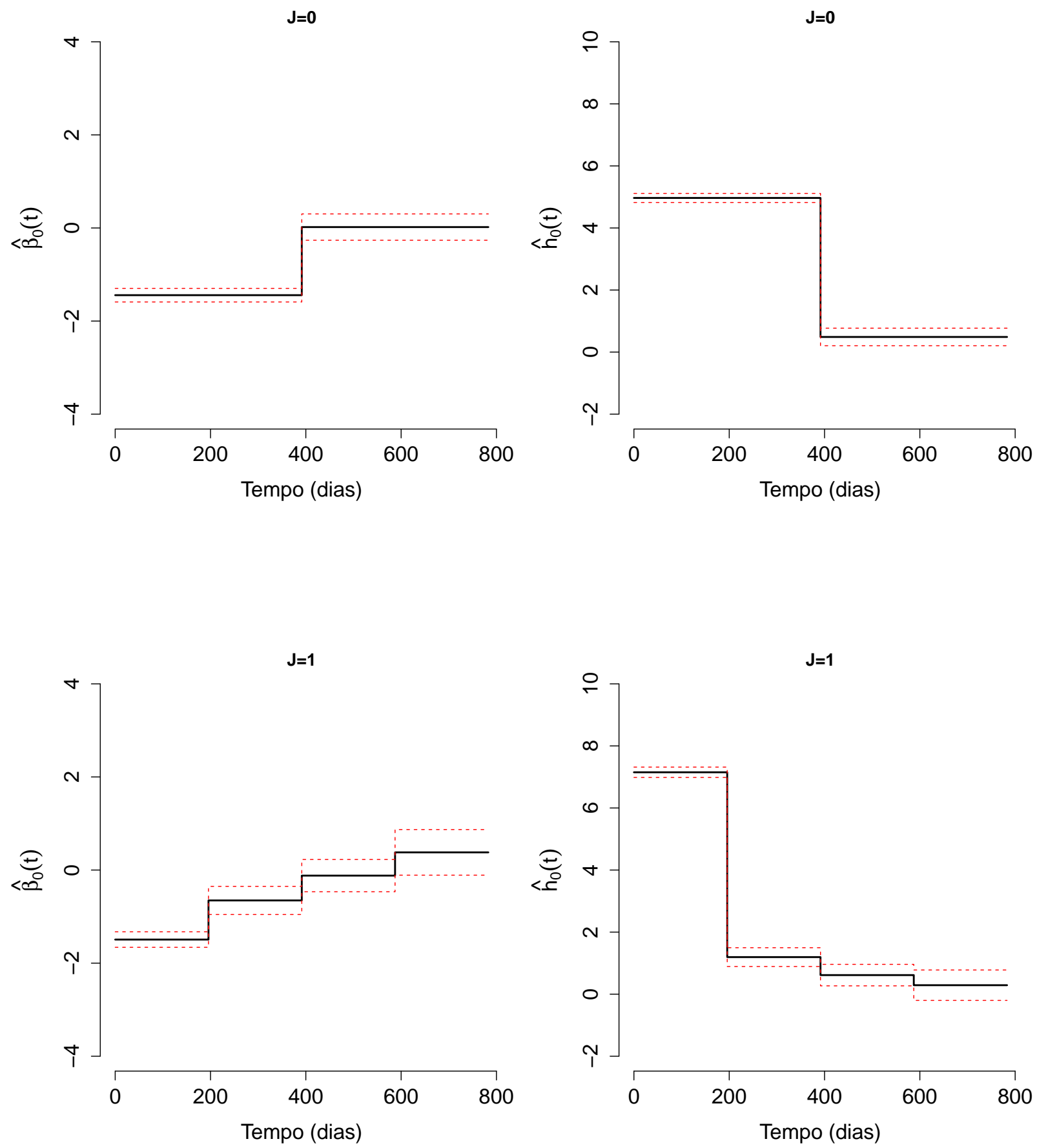

Figura 6.13: Ondaletas clássicas. Estimativa pontual (linha contínua) e intervalo de confiança pontual de $95 \%$ (linha tracejada) para o efeito da cirurgia ao longo do tempo e função de risco basal, respectivamente. 

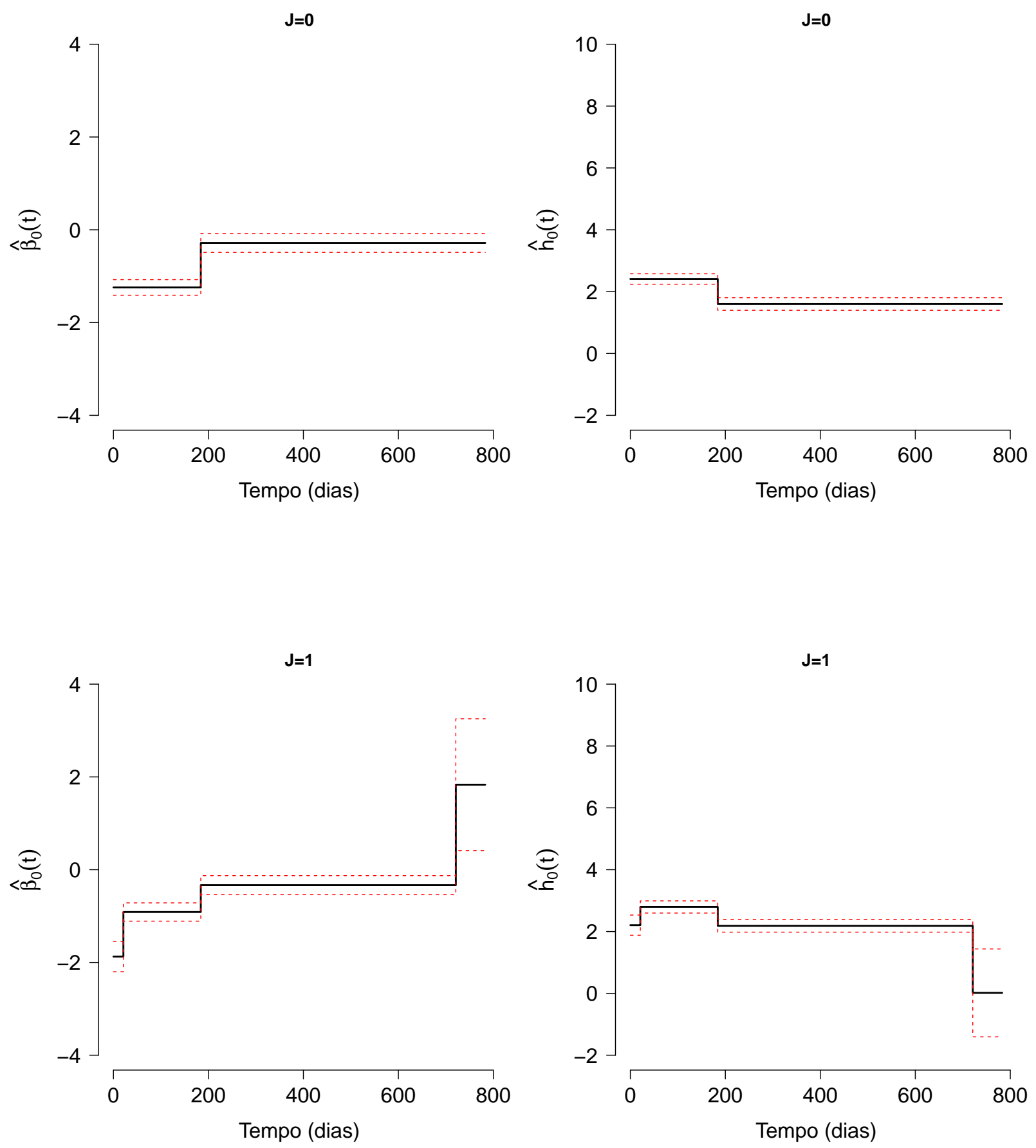

Figura 6.14: Ondaletas deformadas. Estimativa pontual (linha contínua) e intervalo de confiança pontual de $95 \%$ (linha tracejada) para o efeito da cirurgia ao longo do tempo e função de risco basal, respectivamente. 

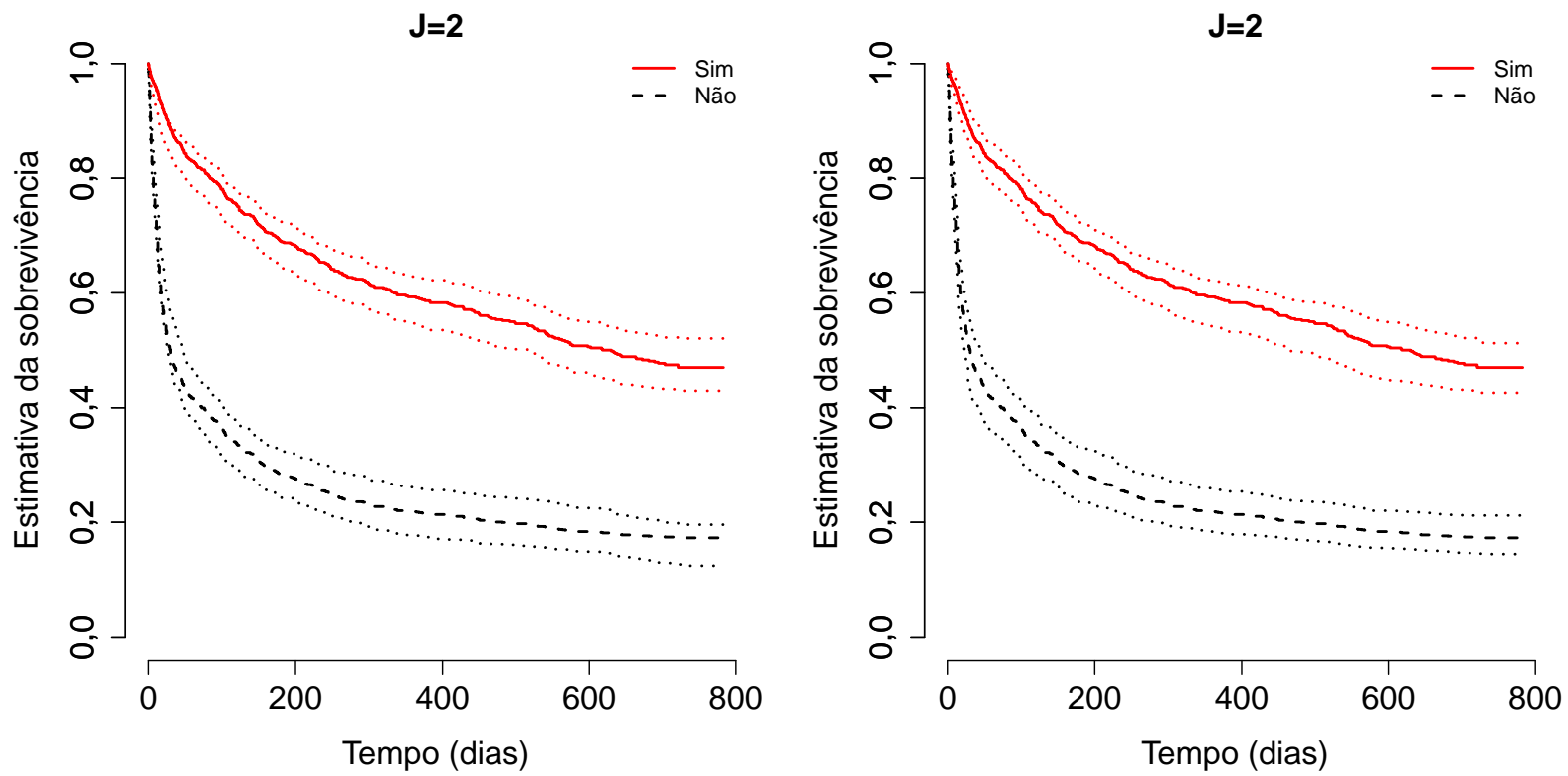

Figura 6.15: Séries de Fourier. Estimativa pontual e intervalo de confiança pontual de 95\% (tracejada) obtido via bootstrap da função de sobrevivência condicional, clássica e deformada, respectivamente.
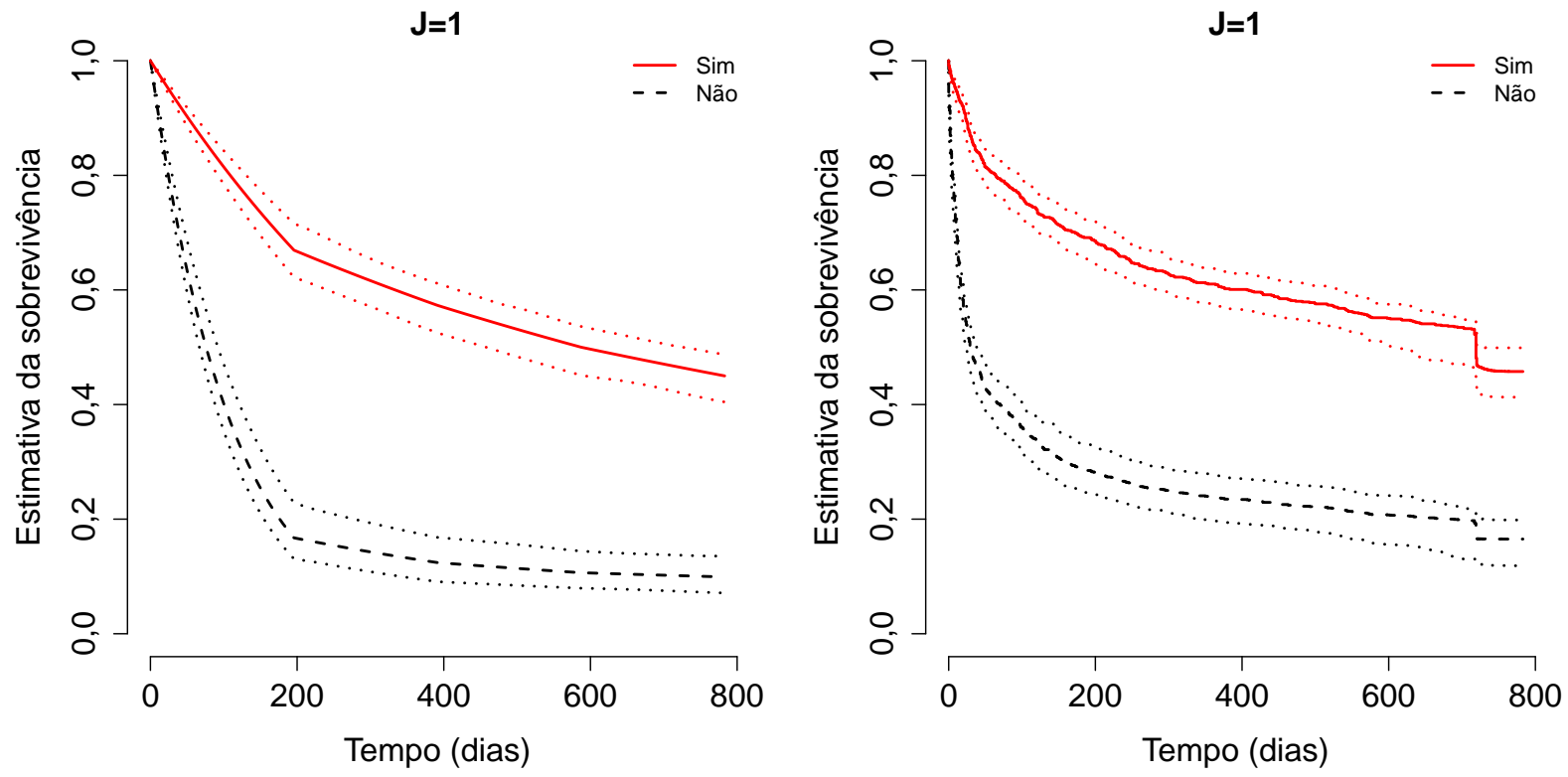

Figura 6.16: Ondaletas Haar. Estimativa pontual e intervalo de confiança pontual de 95\% (linha tracejada) obtido via bootstrap da função de sobrevivência condicional, clássica e deformada, respectivamente. 


\section{Capítulo 7}

\section{Considerações finais}

Neste trabalho, propomos um modelo de regressão tipo Cox em que o coeficiente da regressão é variante no tempo, permitindo assim desvios da proporcionalidade dos riscos para uma dada covariável. Abordamos o problema em dois cenários; semiparamétrico e paramétrico. Consideramos alguns procedimentos de inferência para estimar o efeito da covariável ao longo do tempo e também a função de risco basal via bases de Fourier e ondaletas de Haar. Além disso, para um indivíduo específico, propomos alguns métodos de estimação da função de sobrevivência. Devido à representação do coeficiente dependente do tempo e do risco basal (via séries de Fourier e ondaletas de Haar), por meio de testes de hipóteses, avaliamos se a proporcionalidade dos riscos se sustenta bem como se o risco basal pode ser considerado como constante ao longo do tempo. Estudos de simulações e aplicações a dados reais foram realizados para avaliar o desempenho dos estimadores. Além disso, métodos de seleção de modelos também foram estudados, em que avaliamos via simulações, os modelos mais adequados em cada situação. Procuramos comparar as abordagens propostas aqui com o método mais conhecido e aplicado na prática (baseado nos resíduos de Schoenfeld), e encontramos resultados bem interessantes que reforçam a ideia de que os métodos que propomos podem ser considerados bem competitivos.

As duas abordagens propostas foram comparadas entre si, e pelos estudos de simulação, verificamos que o uso de ondaletas de Haar apresentou melhores ajustes, fornecendo, em geral, os menores RMSE. Observamos ainda uma grande vantagem na escolha das ondaletas quando os critérios de seleção são utilizados. Além disso, os menores RMSE foram obtidos para as ondaletas de Haar. Destacamos ainda que o critério de seleção AIC parece bem indicado na escolha do valor de $J$. Já nas aplicações a dados reais, observamos que para o primeiro conjunto de dados analisado, a abordagem por séries de Fourier se destaca como o melhor modelo ajustado, no entanto as ondaletas de Haar também auxiliaram na interpretação do efeito da covariável ao longo do tempo e na predição da função de sobrevivência para um paciente específico. Para o segundo conjunto de dados, como o procedimento de estimação em duas etapas foi necessário (bases de Fourier), comparamos as abordagens apenas com relação à estimativa baseada nos resíduos de Schoenfeld. Neste cenário, as ondaletas de Haar fornecem os melhores ajustes. Destacamos ainda que a estimação por séries de Fourier e tempos deformados, estimativas instáveis para os tempos finais foram obtidas, inviabilizando assim, utilizar esse procedimento se o interesse estiver nos maiores tempos de vida. Assim como os diversos procedimentos conhecidos na literatura, cada abordagem aqui tem suas peculiaridades, características que devem ser levadas em consideração na escolha do método a ser utilizado.

Uma das principais vantagens em se utilizar as bases de Fourier está no fato de que, em geral, não são necessários muitos coeficientes para se estimar as funções de interesse e, além disso, a curva estimada será sempre contínua e suave. Por outro lado, é localizada apenas em frequência, ao contrário das ondaletas que 
são localizadas em tempo e frequência, simultaneamente.

Considerando o ajuste por meio de ondaletas de Haar, podemos mencionar como vantagem o fato da flexibilidade que a representação em séries de ondaletas apresenta, permitindo a detecção de mudanças abruptas e, em geral, não são necessários valores elevados de $J$ para se obter um bom ajuste, além de apresentar ortogonalidade entre as funções. Por outro lado, citamos como desvantagem o fato de a curva estimada ser constante por partes, apresentado assim pontos de descontinuidades, bem como a necessidade de transformar os tempos observados para o intervalo unitário. A transformação dos tempos foi obtida via o método clássico (dividindo todos os tempos pelo maior tempo observado) e deformado, o qual depende de uma função de distribuição. No entanto, como há uma infinidade de funções de distribuição para deformar os tempos, sua escolha pode conduzir a diferentes estimativas. Nesta tese, a função de distribuição empírica $(F)$ foi considerada devido à semelhança com a função de sobrevivência empírica. É importante ainda mencionar que a perda da ortonormalidade das bases quando se utiliza o método deformado não gera dificuldades ou consequências na estimação dos coeficientes.

Destacamos ainda que se o modelo totalmente paramétrico for empregado, sugerimos utilizar como estimativa inicial no processo de otimização dos parâmetros as estimativas de máxima verossimilhança parcial de $\beta_{0}(t)$ e também se basear na função de risco basal acumulada (obtida pelo estimador de Breslow generalizado). Neste caso, se a estimativa obtida para o risco basal acumulado for próxima de 0 , temos indicativos de problemas de otimização da função de verossimilhança, e nesse caso, tratar o problema em um cenário semiparamétrico pode ser uma alternativa.

É importante mencionar que as estatísticas de teste (Wald, razão de verossimilhanças e escore) foram utilizadas heuristicamente, pois conjecturamos que elas são assintoticamente equivalentes e que sob a hipótese nula, possuem a mesma distribuição. No estudo de simulação, as formas das densidades estimadas sugerem uma aproximação assintótica para a distribuição normal, resultado conhecido para os estimadores de máxima verossimilhança. Assim, as estatísticas de teste foram empregadas supondo que os resultados conhecidos (sob a hipótese nula) também se apliquem aos nossos modelos.

Para a programação dos comandos utilizamos a linguagem R (R Core Team, 2014) em que consideramos a função optim, via o método BFGS, para maximizar as funções de verossimilhança na estimação dos coeficientes. No cenário em que o modelo semiparamétrico ou paramétrico é empregado, as estimativas dos parâmetros foram obtidas rapidamente para níveis baixos $J \in\{0,1\}$, ao passo que para valores elevados $J \in\{2,3\}$, o procedimento de otimização numérica foi relativamente demorado, e, por esse motivo, na maioria dos casos, apenas 100 réplicas foram obtidas nos estudos de simulação.

\subsection{Trabalhos futuros}

Considerando essa linha de pesquisa, temos o interesse em prosseguir nos estudos iniciados aqui. Alguns trabalhos que pretendemos desenvolver posteriormente estão relacionados com a extensão para mais de uma covariável, consistência e normalidade assintótica dos estimadores, bem como obter a distribuição assintótica das estatística de teste de Wald, razão de verossimilhanças e escore (sob a hipótese nula) fazendo o uso da teoria de processos de contagem e martingais. Além disso, temos também o interesse em obter analiticamente as taxas de convergência para a distância média entre os estimadores e as verdadeiras funções, assim como construir bandas de confiança para $\beta_{0}(t), h_{0}(t)$ e demais funções dos tempos. Outro estudo que temos interesse é incorporar a possibilidade de empates nos valores observados, utilizando algumas propostas de modificação na função de verossimilhança parcial. Em um cenário paramétrico em que o risco 
basal é expandido em bases de Fourier, podemos empregar outros métodos numéricos de integração para aproximar a integral definida em (3.17). Devido aos problemas de otimização da função de verossimilhança (cenário paramétrico), pretendemos utilizar outros algoritmos de otimização, como o algoritmos simulated annealing e genético, assim como desenvolver uma abordagem bayesiana dos modelos propostos. Além dessas possibilidades, podem ser alternativas interessantes um estudo utilizando outros tipos de ondaletas, como, ondaletas adaptativas (maior número de coeficientes no início em relação ao final), assim como outras bases. Os polinômios de Legendre podem ser uma alternativa interessante. Pretendemos também incorporar covariáveis dependentes do tempo, assim como considerar no modelo uma mistura de coeficientes invariantes e variantes ao longo do tempo. Pode-se pensar ainda em aplicar esses procedimentos (via séries de Fourier e ondaletas) a outros modelos, como modelos de fragilidade e modelos de risco não proporcional com fração de cura, entre outros. 
CONSIDERAÇÕES FINAIS 


\section{Apêndice A}

\section{Geração de amostras}

Neste apêndice, descrevemos os procedimentos da geração de amostras do modelo de regressão de Cox com o coeficiente variante ao tempo. Inicialmente, apresentamos o cenário em que o coeficiente da regressão é expandido em bases de ondaletas de Haar e nível de resolução $J=0$, e posteriormente o caso em que $\beta_{0}(t)=\sqrt{t}$.

\section{A.1 Expansão em bases de ondaletas de Haar}

Neste primeiro cenário, adotamos uma expansão em bases de ondaletas de Haar para o coeficiente dependente do tempo, de modo que

$$
\beta_{0}(t)=c \phi_{0,0}(t)+d \psi_{0,0}(t)
$$

Observe que essa função pode ser reescrita como

$$
\beta_{0}(t)=\left\{\begin{array}{rr}
c+d, & \text { se } 0 \leq t<0,5 \\
c-d, & \text { se } 0,5 \leq t<1 \\
0, & \text { caso contrário. }
\end{array}\right.
$$

Para a geração dos tempos de vida, utilizamos apenas uma covariável, de forma balanceada, a fim de classificar as unidades amostrais em dois grupos, que pode assumir os valores 0 ou 1 . Além disso, supomos que a função de risco basal é constante ao longo do tempo, $h_{0}(t)=\lambda$. Desta forma, quando $X=0$ o risco condicional é $h(t \mid 0)=\lambda$, se $X=1$ o risco condicional é dado por

$$
h(t \mid 1)=\left\{\begin{array}{rll}
\lambda e^{c+d}, & \text { se } & 0 \leq t<0,5 \\
\lambda e^{c-d}, & \text { se } & 0,5 \leq t<1 \\
\lambda, & \text { se } & t \geq 1
\end{array}\right.
$$

e, consequentemente, a função de risco condicional acumulada é

$$
H(t \mid 1)=\left\{\begin{array}{rll}
\lambda t e^{c+d}, & \text { se } & 0 \leq t<0,5 \\
\frac{\lambda}{2} e^{c+d}+\lambda e^{c-d}\left(t-\frac{1}{2}\right), & \text { se } & 0,5 \leq t<1, \\
\frac{\lambda}{2} e^{c+d}+\frac{\lambda}{2} e^{c-d}+\lambda(t-1), & \text { se } & t \geq 1
\end{array}\right.
$$

Para gerar os conjuntos de dados, devemos seguir as etapas abaixo:

1. Fixar os parâmetros $\lambda, c$ e $d$, de acordo com o comportamento que se deseja.

2. Gerar $n$ (tamanho amostral) valores para a covariável $X$ por meio da distribuição Bernoulli com probabilidade de sucesso igual a 0,5 . 
3. Para observações em que $X=0$, os tempos de falha são gerados da distribuição $T \sim \operatorname{Exp}(\lambda)$, pois $h_{0}(t)=\lambda$ e $S(t)=e^{-\lambda t}$.

4. Para observações em que $X=1$, os tempos de falha são gerados utilizando o método da distribuição inversa. Gere $n$ valores de uma distribuição $\mathrm{U}[0,1]: u_{1}, u_{2}, \ldots, u_{n}$ e assim, $t_{j}^{*}=H^{-1}\left\{\log \left(u_{j}\right)\right\},(j=$ $1, \ldots, n)$, em que

$$
t_{j}^{*}=\left\{\begin{array}{rll}
-\frac{\log \left(u_{j}\right)}{\lambda e^{c+d}}, & \text { se } & 0 \leq-\log \left(u_{j}\right)<\frac{\lambda}{2} e^{c+d}, \\
-\frac{\log \left(u_{j}\right)}{\lambda \lambda e^{c-d}}-\frac{1}{2}\left(e^{2 d}-1\right), & \text { se } & \frac{\lambda}{2} e^{c+d} \leq-\log \left(u_{j}\right)<\frac{\lambda}{2}\left(e^{2 d}+1\right) e^{c-d}, \\
1-\frac{\log \left(u_{j}\right)}{\lambda}-\frac{1}{2}\left(e^{c+d}+e^{c-d}\right), & \text { se } & -\log \left(u_{j}\right) \geq \frac{\lambda}{2}\left(e^{2 d}+1\right) e^{c-d}
\end{array}\right.
$$

Os tempos de censura são gerados considerando o mecanismo de censura aleatória.

5. Quando $X=0$, temos $T \sim \operatorname{Exp}(\lambda)$ e o tempo de censura é gerado da distribuição uniforme, $C \sim \mathrm{U}[0, \tau]$, em que $\tau$ controla a proporção de censura na amostra.

Seja $p_{c}$ o percentual de censura na amostra que será utilizado na geração dos dados. Então,

$$
\begin{aligned}
p_{c}=P(T>C) & =P(C<T)=\mathbb{E}\{P(C<T \mid T=t)\} \\
& =\int_{0}^{\infty} P(C<T \mid T=t) f_{T}(t) d t=\int_{0}^{\tau} \frac{t}{\tau} f_{T}(t) d t+\int_{\tau}^{\infty} f_{T}(t) d t \\
& =\frac{1}{\lambda \tau}\left(1-e^{-\lambda \tau}\right),
\end{aligned}
$$

em que $f_{T}(\cdot)$ representa a função distribuição da variável aleatória $T$.

O valor de $\tau$ é encontrado resolvendo a equação não-linear (A.1). Para encontrar a raiz da equação o método da bissecção (Ruggiero e Lopes, 1997) é utilizado.

No caso em que $X=1$, o tempo de censura é gerado da distribuição uniforme e o parâmetro $\tau$ é ajustado de modo que se tenha o percentual de censura desejado na amostra.

Os dados observados consistem em $t_{j}=\min \left\{t_{j}^{*}, c_{j}\right\}, x_{j}$ e $\delta_{j}=\mathbb{I}\left(t_{j}^{*} \leq c_{j}\right),(j=1, \ldots, n)$.

\section{A.2 Função contínua e crescente}

Agora, consideramos o caso em que o coeficiente da regressão é uma função contínua e crescente, dada por

$$
\beta_{0}(t)=\sqrt{t}
$$

Para a geração dos dados, adotamos uma única covariável $X$ assumindo os valores 0 ou 1. Supomos ainda que a função de risco basal é constante, $h_{0}(t)=\lambda$. Assim, quando $X=0$ a função de risco condicional é $h(t \mid 0)=\lambda$, ao passo que quando $X=1$ o risco condicional é

$$
h(t \mid 1)=\lambda e^{\sqrt{t}}
$$

e, consequentemente, a função de risco condicional acumulada é

$$
H(t \mid 1)=2 \lambda\left\{e^{\sqrt{t}}(\sqrt{t}-1)+1\right\} .
$$

Para gerar os conjuntos de dados, devemos seguir as etapas abaixo:

1. Fixar um valor para $\lambda$;

2. Gerar $n$ (tamanho amostral) valores para a covariável $X$ por meio da distribuição Bernoulli com probabilidade de sucesso igual a 0,5 . 
3. Para observações em que $X=0$, os tempos de falha são gerados da distribuição $T \sim \operatorname{Exp}(\lambda)$.

4. Quando $X=1$, os tempos de falha são gerados utilizando o método da distribuição inversa. Assim, gere $n$ valores de uma distribuição $\mathrm{U}[0,1]: u_{1}, u_{2}, \ldots, u_{n}$ e os tempos de falha $\left(t_{j}^{*}\right)$ são obtidos resolvendo a equação não-linear

$$
\log \left(u_{j}\right)+2 \lambda\left\{e^{\sqrt{t^{*}}}\left(\sqrt{t^{*}}-1\right)+1\right\}=0 .
$$

Os tempos de censura são gerados da distribuição uniforme com parâmetro $\tau$, como realizado anteriormente. Os dados observados consistem em $t_{j}=\min \left\{t_{j}^{*}, c_{j}\right\}, x_{j}$ e $\delta_{j}=\mathbb{I}\left(t_{j}^{*} \leq c_{j}\right),(j=1, \ldots, n)$. 
APÊNDICE A 


\section{Apêndice B}

\section{Matriz de informação observada}

Neste apêndice, apresentamos a matriz de informação observada para os modelos propostos nos Capítulos 3 e 4 .

\section{B.1 Coeficiente dependente do tempo via séries de Fourier}

Seja $\theta=\left(\theta_{1}, \theta_{2}^{\mathrm{T}}\right)^{\mathrm{T}}$ o vetor de parâmetros do modelo (3.10), em que $\theta_{1}=c_{0}$ e $\theta_{2}=\left(c_{1}, \ldots, c_{J}, d_{1}, \ldots, d_{J}\right)^{\mathrm{T}}$. A matriz de informação observada $\mathbf{I}(\beta)$ é dada por

$$
\mathbf{I}(\theta)=-\frac{\partial^{2} \ell(\theta)}{\partial \theta \partial \theta^{\mathrm{T}}}=\left(\begin{array}{ll}
\mathbf{I}_{11} & \mathbf{I}_{12} \\
\mathbf{I}_{12} & \mathbf{I}_{22}
\end{array}\right)
$$

em que

$$
\begin{gathered}
\mathrm{I}_{11}=-\frac{\partial^{2} \ell(\beta)}{\partial \theta_{1}^{2}}, \quad \mathbf{I}_{12}=-\frac{\partial^{2} \ell(\theta)}{\partial \theta_{1} \partial \theta_{2}^{\mathrm{T}}}=-\left(\frac{\partial^{2} \ell(\theta)}{\partial c_{0} \partial c_{1}}, \ldots, \frac{\partial^{2} \ell(\theta)}{\partial c_{0} \partial c_{J}}, \frac{\partial^{2} \ell(\theta)}{\partial c_{0} \partial d_{1}}, \ldots, \frac{\partial^{2} \ell(\theta)}{\partial c_{0} \partial d_{J}}\right)^{\mathrm{T}} \mathrm{e} \\
\mathbf{I}_{22}=-\frac{\partial^{2} \ell(\boldsymbol{\theta})}{\partial \theta_{2} \partial \theta_{2}^{\mathrm{T}}}=-\left(\begin{array}{cccccc}
\frac{\partial^{2} \ell(\boldsymbol{\theta})}{\partial c_{1}^{2}} & \ldots & \frac{\partial^{2} \ell(\boldsymbol{\theta})}{\partial c_{1} \partial c_{J}} & \frac{\partial^{2} \ell(\boldsymbol{\theta})}{\partial c_{1} \partial d_{1}} & \ldots & \frac{\partial^{2} \ell(\boldsymbol{\theta})}{\partial c_{1} \partial d_{J}} \\
\vdots & \ddots & \vdots & \vdots & \ddots & \vdots \\
\frac{\partial^{2} \ell(\boldsymbol{\theta})}{\partial d_{J} \partial c_{1}} & \ldots & \frac{\partial^{2} \ell(\boldsymbol{\theta})}{\partial d_{J} \partial c_{J}} & \frac{\partial^{2} \ell(\boldsymbol{\theta})}{\partial d_{J} \partial d_{1}} & \ldots & \frac{\partial^{2} \ell(\boldsymbol{\theta})}{\partial d_{J}^{2}}
\end{array}\right) .
\end{gathered}
$$

Para a função de verossimilhança parcial (3.11) os elementos da matriz $\mathbf{I}(\theta)$ são dadas por

$$
\begin{aligned}
\frac{\partial^{2} \ell(\theta)}{\partial \theta_{1}^{2}} & =\frac{1}{4} \sum_{i=1}^{n} \delta_{i}\left(\frac{a\left(t_{i}\right) b\left(t_{i}\right)-c\left(t_{i}\right)^{2}}{a\left(t_{i}\right)^{2}}\right), \\
\frac{\partial^{2} \ell(\theta)}{\partial c_{0} \partial c_{k}} & =\frac{1}{2} \sum_{i=1}^{n} \delta_{i}\left(\frac{a\left(t_{i}\right) f\left(t_{i}\right)-e_{k}\left(t_{i}\right) c\left(t_{i}\right)}{a\left(t_{i}\right)^{2}}\right), \\
\frac{\partial^{2} \ell(\theta)}{\partial c_{k} \partial c_{j}} & =\sum_{i=1}^{n} \delta_{i}\left(\frac{a\left(t_{i}\right) d\left(t_{i}\right)-e_{k}\left(t_{i}\right) e_{j}\left(t_{i}\right)}{a\left(t_{i}\right)^{2}}\right), \\
\frac{\partial^{2} \ell(\theta)}{\partial c_{0} \partial d_{k}} & =\frac{1}{2} \sum_{i=1}^{n} \delta_{i}\left(\frac{a\left(t_{i}\right) g\left(t_{i}\right)-h_{k}\left(t_{i}\right) c\left(t_{i}\right)}{a\left(t_{i}\right)^{2}}\right), \\
\frac{\partial^{2} \ell(\theta)}{\partial d_{k} \partial c_{j}} & =\sum_{i=1}^{n} \delta_{i}\left(\frac{a\left(t_{i}\right) i\left(t_{i}\right)-e_{k}\left(t_{i}\right) h_{k}\left(t_{i}\right)}{a\left(t_{i}\right)^{2}}\right)
\end{aligned}
$$

$\mathrm{e}$

$$
\frac{\partial^{2} \ell(\theta)}{\partial d_{k} \partial d_{j}}=\sum_{i=1}^{n} \delta_{i}\left(\frac{a\left(t_{i}\right) j\left(t_{i}\right)-h_{k}\left(t_{i}\right) e_{j}\left(t_{i}\right)}{a\left(t_{i}\right)^{2}}\right),
$$




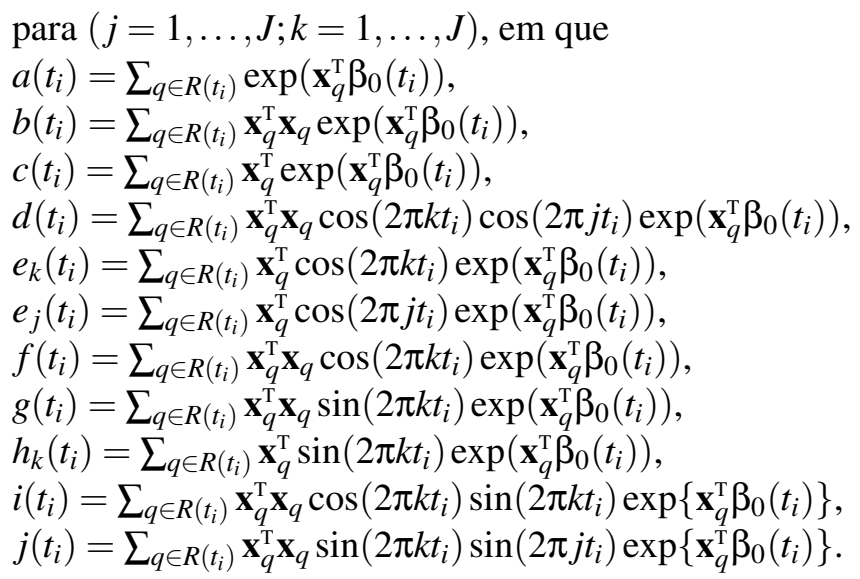

\section{B.2 $\beta_{0}(t)$ e $h_{0}(t)$ via bases de Fourier}

Seja $\theta=\left(\theta_{1}^{\mathrm{T}}, \theta_{2}^{\mathrm{T}}\right)^{\mathrm{T}}$ o vetor de parâmetros do modelo (3.16), em que $\theta_{1}=\left(a_{0}, c_{0}\right)^{\mathrm{T}}$ e $\theta_{2}=\left(a_{1}, \ldots, a_{J}, b_{1}, \ldots, b_{J}, c_{1}, \ldots, c_{J}, d_{1}, \ldots, d_{J}\right)^{\mathrm{T}}$. A matriz de informação observada $\mathbf{I}(\theta)$ é dada por

$$
\mathbf{I}(\boldsymbol{\theta})=-\frac{\partial^{2} \ell(\boldsymbol{\theta})}{\partial \theta \partial \theta^{\mathrm{T}}}=\left(\begin{array}{ll}
\mathbf{I}_{11} & \mathbf{I}_{12} \\
\mathbf{I}_{12} & \mathbf{I}_{22}
\end{array}\right),
$$

em que

$$
\begin{aligned}
& \mathbf{I}_{11}=-\frac{\partial^{2} \ell(\boldsymbol{\theta})}{\partial \theta_{1} \partial \theta_{1}^{T}}=-\left(\begin{array}{ll}
\frac{\partial^{2} \ell(\boldsymbol{\theta})}{\partial a_{0}^{2}} & \frac{\partial^{2} \ell(\boldsymbol{\theta})}{\partial a_{0} \partial c_{0}} \\
\frac{\partial^{2} \ell(\boldsymbol{\theta})}{\partial c_{0} \partial a_{0}} & \frac{\partial^{2} \ell(\boldsymbol{\theta})}{\partial c_{0}^{2}}
\end{array}\right)
\end{aligned}
$$

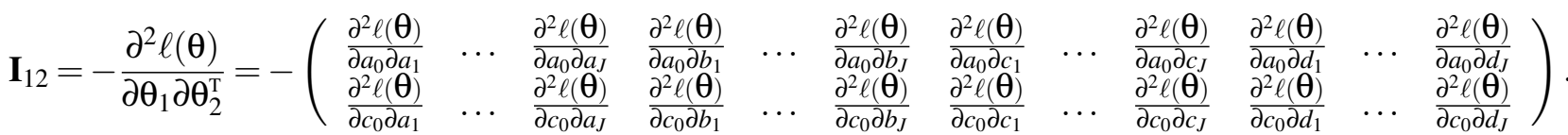

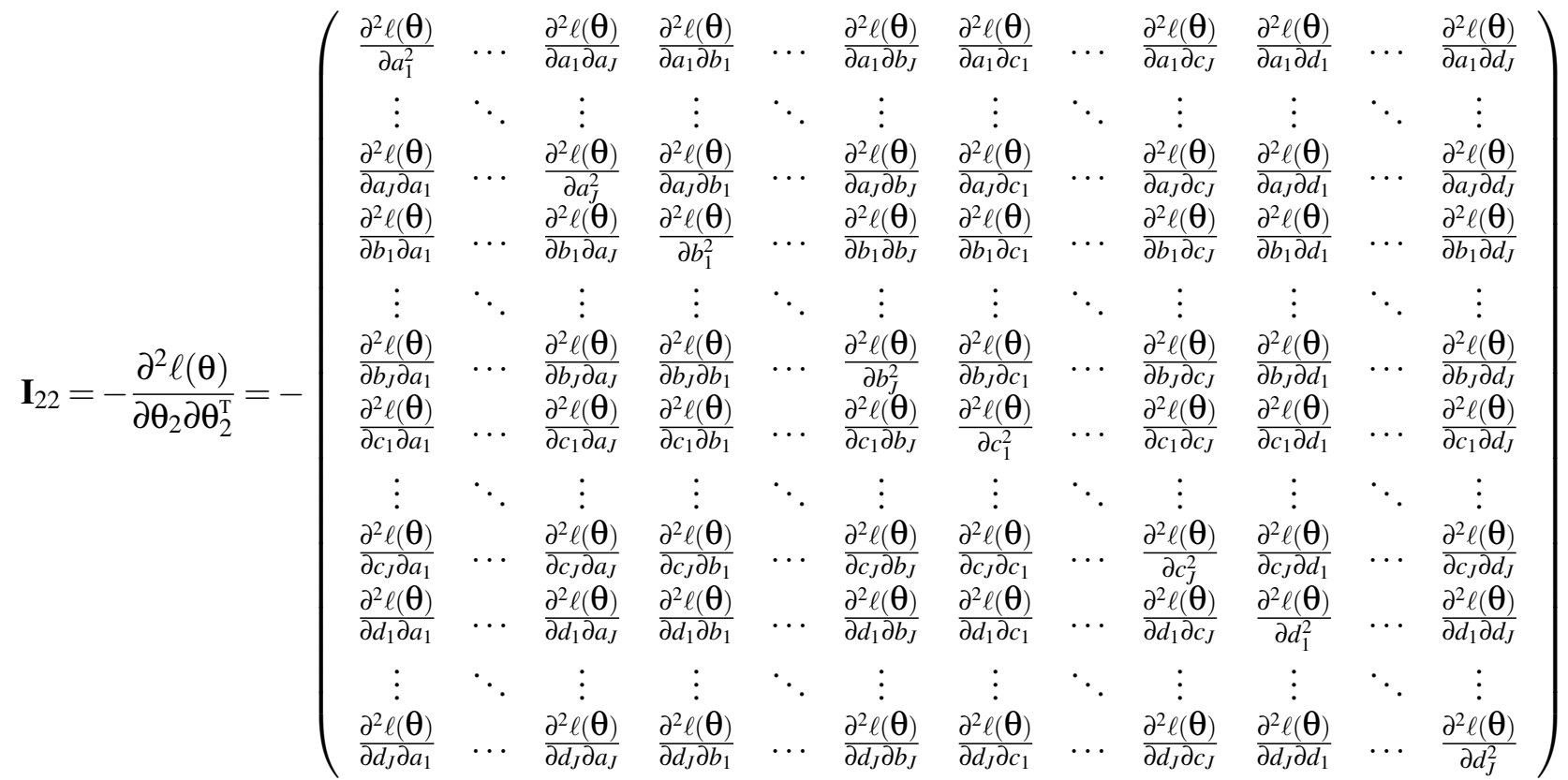


Para a função de verossimilhança parcial (3.17,) os elementos da matriz $\mathbf{I}(\theta)$ são dadas por

$$
\begin{aligned}
\frac{\partial^{2} \ell(\theta)}{\partial a_{0}^{2}} & =\frac{1}{4} \sum_{i=1}^{n} \frac{\delta_{i}}{h_{0}\left(t_{i}\right)}, \\
\frac{\partial^{2} \ell(\theta)}{\partial a_{0} \partial a_{k}} & =\frac{1}{2} \sum_{i=1}^{n} \frac{\delta_{i} \cos \left(2 \pi k t_{i}\right)}{h_{0}^{2}\left(t_{i}\right)}+\frac{\partial^{2}}{\partial a_{0} \partial a_{k}} \sum_{i=1}^{n} \int_{0}^{t_{i}} h_{0}(s) \exp \left(x_{i} \beta_{0}(s)\right) d s, \\
\frac{\partial^{2} \ell(\theta)}{\partial a_{0} \partial b_{k}} & =\frac{1}{2} \sum_{i=1}^{n} \frac{\delta_{i} \sin \left(2 \pi k t_{i}\right)}{h_{0}^{2}\left(t_{i}\right)}+\frac{\partial^{2}}{\partial a_{0} \partial b_{k}} \sum_{i=1}^{n} \int_{0}^{t_{i}} h_{0}(s) \exp \left(x_{i} \beta_{0}(s)\right) d s, \\
\frac{\partial^{2} \ell(\theta)}{\partial a_{j} \partial a_{k}} & =\sum_{i=1}^{n} \frac{\delta_{i} \cos \left(2 \pi k t_{i}\right) \cos \left(2 \pi j t_{i}\right)}{h_{0}^{2}\left(t_{i}\right)}+\frac{\partial^{2}}{\partial a_{j} \partial a_{k}} \sum_{i=1}^{n} \int_{0}^{t_{i}} h_{0}(s) \exp \left(x_{i} \beta_{0}(s)\right) d s, \\
\frac{\partial^{2} \ell(\theta)}{\partial a_{k} \partial b_{k}} & =\sum_{i=1}^{n} \frac{\delta_{i} \sin \left(2 \pi k t_{i}\right) \cos \left(2 \pi k t_{i}\right)}{h_{0}^{2}\left(t_{i}\right)}+\frac{\partial^{2}}{\partial a_{k} \partial b_{k}} \sum_{i=1}^{n} \int_{0}^{t_{i}} h_{0}(s) \exp \left(x_{i} \beta_{0}(s)\right) d s, \\
\frac{\partial^{2} \ell(\theta)}{\partial b_{j} \partial b_{k}} & =\sum_{i=1}^{n} \frac{\delta_{i} \sin \left(2 \pi k t_{i}\right) \sin \left(2 \pi j t_{i}\right)}{h_{0}^{2}\left(t_{i}\right)}+\frac{\partial^{2}}{\partial b_{j} \partial b_{k}} \sum_{i=1}^{n} \int_{0}^{t_{i}} h_{0}(s) \exp \left(x_{i} \beta_{0}(s)\right) d s, \\
\frac{\partial^{2} \ell(\theta)}{\partial c_{0}^{2}} & =\frac{\partial^{2}}{\partial c_{0}^{2}} \sum_{i=1}^{n} \int_{0}^{t_{i}} h_{0}(s) \exp \left(x_{i} \beta_{0}(s)\right) d s, \\
\frac{\partial^{2} \ell(\theta)}{\partial c_{0} \partial c_{k}} & =\frac{\partial^{2}}{\partial c_{0} \partial c_{k}} \sum_{i=1}^{n} \int_{0}^{t_{i}} h_{0}(s) \exp \left(x_{i} \beta_{0}(s)\right) d s, \\
\frac{\partial^{2} \ell(\theta)}{\partial c_{0} \partial d_{k}} & =\frac{\partial^{2}}{\partial c_{0} \partial d_{k}} \sum_{i=1}^{n} \int_{0}^{t_{i}} h_{0}(s) \exp \left(x_{i} \beta_{0}(s)\right) d s, \\
\frac{\partial^{2} \ell(\theta)}{\partial c_{j} \partial c_{k}} & =\frac{\partial^{2}}{\partial c_{j} \partial c_{k}} \sum_{i=1}^{n} \int_{0}^{t_{i}} h_{0}(s) \exp \left(x_{i} \beta_{0}(s)\right) d s, \\
\frac{\partial^{2} \ell(\theta)}{\partial c_{k} \partial d_{k}} & =\frac{\partial^{2}}{\partial c_{k} \partial d_{k}} \sum_{i=1}^{n} \int_{0}^{t_{i}} h_{0}(s) \exp \left(x_{i} \beta_{0}(s)\right) d s
\end{aligned}
$$

$\mathrm{e}$

$$
\frac{\partial^{2} \ell(\theta)}{\partial d_{j} \partial d_{k}}=\frac{\partial^{2}}{\partial d_{j} \partial d_{k}} \sum_{i=1}^{n} \int_{0}^{t_{i}} h_{0}(s) \exp \left(x_{i} \beta_{0}(s)\right\} d s
$$

para $(j=1, \ldots, J ; k=1, \ldots, J)$, em que

$$
h_{0}(s)=\frac{a_{0}}{2}+\sum_{k=1}^{J}\left\{a_{k} \cos (2 \pi k s)+b_{k} \sin (2 \pi k s)\right\} \quad \text { e } \quad \beta_{0}(s)=\frac{c_{0}}{2}+\sum_{k=1}^{J}\left\{c_{k} \cos (2 \pi k s)+d_{k} \sin (2 \pi k s)\right\} .
$$

\section{B.3 Coeficiente dependente do tempo via bases de ondaletas Haar}

Seja $\theta=\left(\theta_{1}, \theta_{2}^{\mathrm{T}}\right)^{\mathrm{T}}$ o vetor de parâmetros do modelo (4.1), em que $\theta_{1}=c$ e $\theta_{2}=\left(d_{0,0}, \ldots, d_{J, 2^{J}-1}\right)^{\mathrm{T}}$. A matriz de informação observada $\mathbf{I}(\theta)$ é dada por

$$
\mathbf{I}(\theta)=-\frac{\partial^{2} \ell(\theta)}{\partial \theta \partial \theta^{T}}=\left(\begin{array}{ll}
\mathbf{I}_{11} & \mathbf{I}_{12} \\
\mathbf{I}_{12} & \mathbf{I}_{22}
\end{array}\right)
$$

em que

$$
\mathrm{I}_{11}=-\frac{\partial^{2} \ell(\boldsymbol{\theta})}{\partial \theta_{1}^{2}}, \quad \mathbf{I}_{12}=-\frac{\partial^{2} \ell(\boldsymbol{\theta})}{\partial \theta_{1} \partial \theta_{2}^{\mathrm{T}}}=-\left(\frac{\partial^{2} \ell(\theta)}{\partial c \partial d_{0,0}}, \ldots, \frac{\partial^{2} \ell(\theta)}{\partial c \partial d_{J, 2^{J}-1}}\right)^{\mathrm{T}} \quad \mathrm{e}
$$




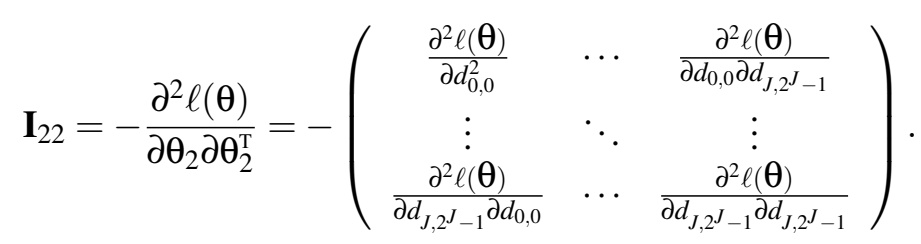

Para a função de verossimilhança parcial (4.2) os elementos da matriz $\mathbf{I}(\theta)$ são dadas por

$$
\begin{aligned}
\frac{\partial^{2} \ell(\theta)}{\partial \theta_{1}^{2}} & =\sum_{i=1}^{n} \delta_{i}\left(\frac{a\left(t_{i}\right) b\left(t_{i}\right)-c\left(t_{i}\right)^{2}}{a\left(t_{i}\right)^{2}}\right), \\
\frac{\partial^{2} \ell(\theta)}{\partial c \partial d_{j, k}} & =\sum_{i=1}^{n} \delta_{i}\left(\frac{a\left(t_{i}\right) d_{j, k}\left(t_{i}\right)-c\left(t_{i}\right) e_{j, k}\left(t_{i}\right)}{a\left(t_{i}\right)^{2}}\right)
\end{aligned}
$$

$\mathrm{e}$

$$
\frac{\partial^{2} \ell(\theta)}{\partial d_{j, k} \partial d_{l, m}}=\sum_{i=1}^{n} \delta_{i}\left\{\frac{g_{j, k, l, m}\left(t_{i}\right) a\left(t_{i}\right)-e_{j, k}\left(t_{i}\right) e_{l, m}\left(t_{i}\right)}{a\left(t_{i}\right)^{2}}\right\}
$$

para $\left(j, l=0, \ldots, J ; k, m=0, \ldots, 2^{J}-1\right)$, em que

$a\left(t_{i}\right)=\sum_{q \in R\left(t_{i}\right)} \exp \left(\mathbf{x}_{q}^{\mathrm{T}} \beta_{0}\left(t_{i}\right)\right)$,

$b\left(t_{i}\right)=\sum_{q \in R\left(t_{i}\right)} \mathbf{x}_{q}^{\mathrm{T}} \mathbf{x}_{q}\left\{\phi_{0,0}\left(t_{i}\right)\right\}^{2} \exp \left(\mathbf{x}_{q}^{\mathrm{T}} \beta_{0}\left(t_{i}\right)\right)$,

$c\left(t_{i}\right)=\sum_{q \in R\left(t_{i}\right)} \mathbf{x}_{q}^{\mathrm{T}} \phi_{0,0}\left(t_{i}\right) \exp \left(\mathbf{x}_{q}^{\mathrm{T}} \beta_{0}\left(t_{i}\right)\right)$,

$d_{j, k}\left(t_{i}\right)=\sum_{q \in R\left(t_{i}\right)} \mathbf{x}_{q}^{\mathrm{T}} \mathbf{x}_{q} \phi_{0,0}\left(t_{i}\right) \psi_{j, k}\left(t_{i}\right) \exp \left(\mathbf{x}_{q}^{\mathrm{T}} \beta_{0}\left(t_{i}\right)\right)$,

$e_{j, k}\left(t_{i}\right)=\sum_{q \in R\left(t_{i}\right)} \mathbf{x}_{q}^{\mathrm{T}} \psi_{j, k}\left(t_{i}\right) \exp \left(\mathbf{x}_{q}^{\mathrm{T}} \beta_{0}\left(t_{i}\right)\right)$,

$f_{j, k}\left(t_{i}\right)=\sum_{q \in R\left(t_{i}\right)} \mathbf{x}_{q}^{\mathrm{T}} \mathbf{x}_{q}\left\{\psi_{j, k}\left(t_{i}\right)\right\}^{2} \exp \left(\mathbf{x}_{q}^{\mathrm{T}} \beta_{0}\left(t_{i}\right)\right)$,

$g_{j, k, l, m}\left(t_{i}\right)=\sum_{q \in R\left(t_{i}\right)} \mathbf{x}_{q}^{\mathrm{T}} \mathbf{x}_{q} \psi_{j, k}\left(t_{i}\right) \psi_{l, m}\left(t_{i}\right) \exp \left(\mathbf{x}_{q}^{\mathrm{T}} \beta_{0}\left(t_{i}\right)\right)$.

\section{B.4 $\beta_{0}(t)$ e $h_{0}(t)$ via bases de ondaletas Haar}

Seja $\theta=\left(\theta_{1}^{\mathrm{T}}, \theta_{2}^{\mathrm{T}}\right)^{\mathrm{T}}$ o vetor de parâmetros do modelo (4.9), em que $\theta_{1}=(a, c)^{\mathrm{T}}$ e $\theta_{2}=\left(b_{0,0}, \ldots, b_{J, 2^{J}-1}, d_{0,0}, \ldots, d_{J, 2^{J}-1}\right)^{\mathrm{T}}$. A matriz de informação observada $\mathbf{I}(\theta)$ é dada por

$$
\mathbf{I}(\theta)=-\frac{\partial^{2} \ell(\theta)}{\partial \theta \partial \theta^{\mathrm{T}}}=\left(\begin{array}{ll}
\mathbf{I}_{11} & \mathbf{I}_{12} \\
\mathbf{I}_{12} & \mathbf{I}_{22}
\end{array}\right),
$$

em que

$$
\begin{aligned}
& \mathbf{I}_{11}=-\frac{\partial^{2} \ell(\theta)}{\partial \theta_{1} \partial \theta_{1}^{\mathrm{T}}}=-\left(\begin{array}{cc}
\frac{\partial^{2} \ell(\boldsymbol{\theta})}{\partial a^{2}} & \frac{\partial^{2} \ell(\boldsymbol{\theta})}{\partial \partial \partial c} \\
\frac{\partial^{2} \ell(\boldsymbol{\theta})}{\partial c \partial a} & \frac{\partial^{2} \ell(\boldsymbol{\theta})}{\partial c^{2}}
\end{array}\right) \\
& \mathbf{I}_{12}=-\frac{\partial^{2} \ell(\boldsymbol{\theta})}{\partial \theta_{1} \partial \theta_{2}^{\mathrm{T}}}=-\left(\begin{array}{cccccc}
\frac{\partial^{2} \ell(\boldsymbol{\theta})}{\partial a \partial b_{0,0}} & \cdots & \frac{\partial^{2} \ell(\boldsymbol{\theta})}{\partial a \partial b_{J, 2 J}-1} & \frac{\partial^{2} \ell(\boldsymbol{\theta})}{\partial a \partial d_{0,0}} & \cdots & \frac{\partial^{2} \ell(\boldsymbol{\theta})}{\partial a \partial d_{J, 2 J} J^{\prime}} \\
\frac{\partial^{2} \ell(\boldsymbol{\theta})}{\partial c \partial b_{0,0}} & \cdots & \frac{\partial^{2} \ell(\boldsymbol{\theta})}{\partial c \partial b_{J, 2} J_{-1}} & \frac{\partial^{2} \ell(\boldsymbol{\theta})}{\partial c \partial d_{0,0}} & \cdots & \frac{\partial^{2} \ell(\boldsymbol{\theta})}{\partial c \partial d_{J, 2} J_{-1}}
\end{array}\right) .
\end{aligned}
$$

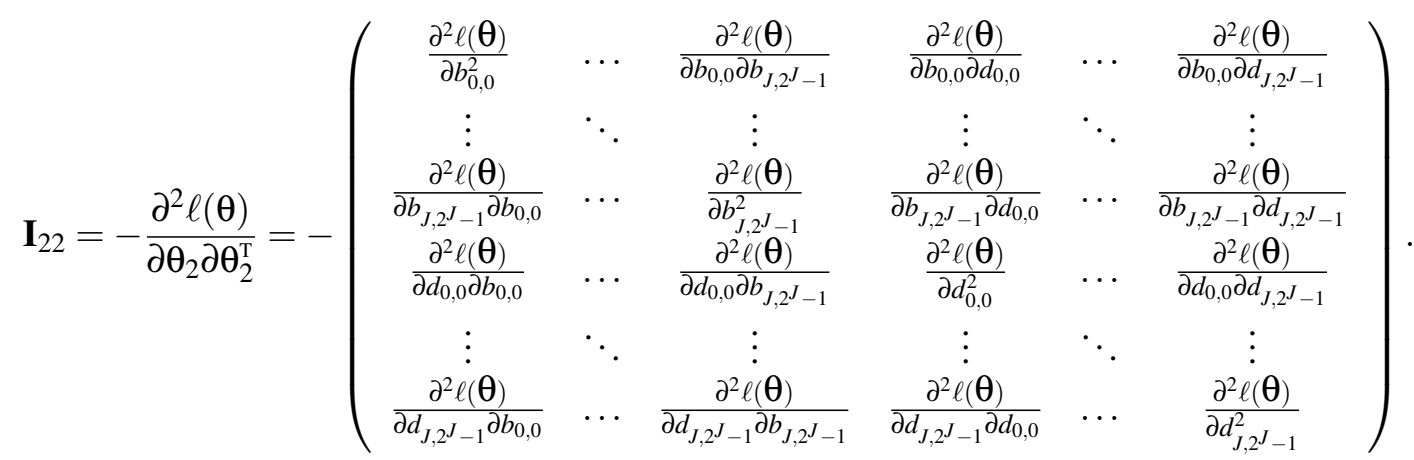


Para a função de verossimilhança parcial (4.10) os elementos da matriz $\mathbf{I}(\theta)$ são dadas por

$$
\begin{aligned}
\frac{\partial^{2} \ell(\theta)}{\partial a^{2}} & =\sum_{i=1}^{n} \frac{\delta_{i} \phi_{0,0}^{2}\left(t_{i}\right)}{h_{0}^{2}\left(t_{i}\right)} \\
\frac{\partial^{2} \ell(\theta)}{\partial a \partial b_{j, k}} & =\sum_{i=1}^{n} \frac{\delta_{i} \phi_{0,0}\left(t_{i}\right) \psi_{j, k}\left(t_{i}\right)}{h_{0}^{2}\left(t_{i}\right)}+\frac{\partial^{2}}{\partial a \partial b_{j, k}} \sum_{i=1}^{n} \int_{0}^{t_{i}} h_{0}(s) \exp \left(x_{i} \beta_{0}(s)\right) d s, \\
\frac{\partial^{2} \ell(\theta)}{\partial a \partial c} & =\frac{\partial^{2}}{\partial a \partial c} \sum_{i=1}^{n} \int_{0}^{t_{i}} h_{0}(s) \exp \left(x_{i} \beta_{0}(s)\right) d s, \\
\frac{\partial^{2} \ell(\theta)}{\partial a \partial d_{j, k}} & =\frac{\partial^{2}}{\partial a \partial d_{j, k}} \sum_{i=1}^{n} \int_{0}^{t_{i}} h_{0}(s) \exp \left(x_{i} \beta_{0}(s)\right) d s, \\
\frac{\partial^{2} \ell(\theta)}{\partial c^{2}} & =\frac{\partial^{2}}{\partial c^{2}} \sum_{i=1}^{n} \int_{0}^{t_{i}} h_{0}(s) \exp \left(x_{i} \beta_{0}(s)\right\} d s, \\
\frac{\partial^{2} \ell(\theta)}{\partial c \partial b_{j, k}} & =\frac{\partial^{2}}{\partial c \partial b_{j, k}} \sum_{i=1}^{n} \int_{0}^{t_{i}} h_{0}(s) \exp \left(x_{i} \beta_{0}(s)\right) d s, \\
\frac{\partial^{2} \ell(\theta)}{\partial c \partial d_{j, k}} & =\frac{\partial^{2}}{\partial c \partial d_{j, k}} \sum_{i=1}^{n} \int_{0}^{t_{i}} h_{0}(s) \exp \left(x_{i} \beta_{0}(s)\right) d s, \\
\frac{\partial^{2} \ell(\theta)}{\partial b_{j, k}^{2}} & =\frac{\partial^{2}}{\partial b_{j, k}^{2}} \sum_{i=1}^{n} \int_{0}^{t_{i}} h_{0}(s) \exp \left(x_{i} \beta_{0}(s)\right) d s, \\
\frac{\partial^{2} \ell(\theta)}{\partial b_{j, k} \partial d_{j, k}} & =\frac{\partial^{2}}{\partial b_{j, k} \partial d_{j, k}} \sum_{i=1}^{n} \int_{0}^{t_{i}} h_{0}(s) \exp \left(x_{i} \beta_{0}(s)\right) d s, \\
\frac{\partial^{2} \ell(\theta)}{\partial d_{j, k} \partial d_{l, m}} & =\frac{\partial^{2}}{\partial d_{j, k} \partial d_{l, m}} \sum_{i=1}^{n} \int_{0}^{t_{i}} h_{0}(s) \exp \left(x_{i} \beta_{0}(s)\right) d s, \\
\frac{\partial^{2} \ell(\theta)}{\partial b_{j, k} \partial b_{l, m}} & =\sum_{i=1}^{n} \frac{\delta_{i} \psi_{j, k}\left(t_{i}\right) \Psi_{l, m}\left(t_{i}\right)}{h_{0}^{2}\left(t_{i}\right)}+\frac{\partial^{2}}{\partial b_{j, k} \partial b_{l, m}} \sum_{i=1}^{n} \int_{0}^{t_{i}} h_{0}(s) \exp \left(x_{i} \beta_{0}(s)\right) d s
\end{aligned}
$$

$\mathrm{e}$

$$
\frac{\partial^{2} \ell(\theta)}{\partial d_{j, k}^{2}}=\frac{\partial^{2}}{\partial d_{j, k}^{2}} \sum_{i=1}^{n} \int_{0}^{t_{i}} h_{0}(s) \exp \left(x_{i} \beta_{0}(s)\right) d s,
$$

para $\left(j, l=0, \ldots, J ; k, m=0, \ldots, 2^{J}-1\right)$, em que

$$
h_{0}(s)=a \phi_{0,0}(s)+\sum_{j=0}^{J} \sum_{k=0}^{2^{j}-1} b_{j, k} \psi_{j, k}(s) \quad \text { e } \quad \beta_{0}(s)=c \phi_{0,0}(s)+\sum_{j=0}^{J} \sum_{k=0}^{2^{j}-1} d_{j, k} \psi_{j, k}(s) .
$$

No caso específico em que o nível de resolução adotado é $J=0$, a função de risco basal e o coeficiente dependente do tempo são, respectivamente,

$$
h_{0}(s)=a \phi_{0,0}(s)+b_{0,0} \psi_{0,0}(s) \quad \text { e } \quad \beta_{0}(s)=c \phi_{0,0}(s)+d_{0,0} \psi_{0,0}(s)
$$

e os elementos da matriz de informação observada são

$$
\begin{aligned}
\frac{\partial^{2} \ell(\theta)}{\partial a^{2}} & =\frac{\partial^{2} \ell(\theta)}{\partial b_{0,0}^{2}}=\sum_{i=1}^{n} \frac{\delta_{i} \phi_{0,0}^{2}\left(t_{i}\right)}{h_{0}^{2}\left(t_{i}\right)}, \\
\frac{\partial^{2} \ell(\theta)}{\partial a \partial b_{0,0}} & =\sum_{i=1}^{n} \frac{\delta_{i} \phi_{0,0}\left(t_{i}\right) \psi_{0,0}\left(t_{i}\right)}{h_{0}^{2}\left(t_{i}\right)}, \\
\frac{\partial^{2} \ell(\theta)}{\partial a \partial c} & =\sum_{I_{1}} t_{i} x_{i} \exp \left(x_{i}\left(c+d_{0,0}\right)\right)+\frac{1}{2} \sum_{I_{2}} x_{i} \exp \left(x_{i}\left(c+d_{0,0}\right)\right)+\sum_{I_{2}} x_{i}\left(t_{i}-0,5\right) \exp \left(x_{i}\left(c-d_{0,0}\right)\right),
\end{aligned}
$$




$$
\begin{aligned}
\frac{\partial^{2} \ell(\theta)}{\partial a \partial d_{0,0}}=\frac{\partial^{2} \ell(\theta)}{\partial c \partial b_{0,0}}= & \sum_{I_{1}} t_{i} x_{i} \exp \left(x_{i}\left(c+d_{0,0}\right)\right)+\frac{1}{2} \sum_{I_{2}} x_{i} \exp \left(x_{i}\left(c+d_{0,0}\right)\right) \\
& -\sum_{I_{2}} x_{i}\left(t_{i}-0,5\right) \exp \left(x_{i}\left(c-d_{0,0}\right)\right), \\
\frac{\partial^{2} \ell(\theta)}{\partial c^{2}}=\frac{\partial^{2} \ell(\theta)}{\partial d_{0}^{2}}= & \sum_{I_{1}} t_{i} x_{i}^{2}\left(a+b_{0,0}\right) \exp \left(x_{i}\left(c+d_{0,0}\right)\right)+\frac{1}{2} \sum_{I_{2}} x_{i}^{2}\left(a+b_{0,0}\right) \exp \left(x_{i}\left(c+d_{0,0}\right)\right) \\
& +\sum_{I_{2}} x_{i}^{2}\left(t_{i}-0,5\right)\left(a-b_{0,0}\right) \exp \left(x_{i}\left(c-d_{0,0}\right)\right), \\
\frac{\partial^{2} \ell(\theta)}{\partial c \partial d_{0,0}}= & \sum_{I_{1}} t_{i} x_{i}^{2}\left(a+b_{0,0}\right) \exp \left(x_{i}\left(c+d_{0,0}\right)\right)+\frac{1}{2} \sum_{I_{2}} x_{i}^{2}\left(a+b_{0,0}\right) \exp \left(x_{i}\left(c+d_{0,0}\right)\right) \\
& -\sum_{I_{2}} x_{i}^{2}\left(t_{i}-0,5\right)\left(a-b_{0,0}\right) \exp \left(x_{i}\left(c-d_{0,0}\right)\right)
\end{aligned}
$$

e

$$
\frac{\partial^{2} \ell(\theta)}{\partial b_{0,0} \partial d_{0,0}}=\sum_{I_{1}} t_{i} x_{i} \exp \left(x_{i}\left(c+d_{0,0}\right)\right)+\frac{1}{2} \sum_{I_{2}} x_{i} \exp \left(x_{i}\left(c+d_{0,0}\right)\right)+\sum_{I_{2}} x_{i}\left(t_{i}-0,5\right) \exp \left(x_{i}\left(c-d_{0,0}\right)\right),
$$

em que

$$
I_{1}=\left\{i: 0 \leq t_{i}<0,5\right\} \quad \text { e } \quad I_{2}=\left\{i: 0,5 \leq t_{i}<1\right\}
$$




\title{
Referências Bibliográficas
}

\begin{abstract}
Abrahamowicz et al. (1996) Michal Abrahamowicz, Todd Mackenzie e John M. Esdaile. Time-dependent hazard ratio: modeling and hypothesis testing with application in lupus nephritis. Journal of the American Statistical Association, 91:1432-1439. Citado na pág. 1, 45
\end{abstract}

Akaike (1974) Hirotugu Akaike. A new look at the statistical model identification. IEEE Transactions on Automatic Control, 19:716-723. Citado na pág. 45

Andersen e Gill (1982) Per Kragh Andersen e Richard D. Gill. Cox's regression model for counting processes: A large sample study. The Annals of Statistics, 10:1100-1120. Citado na pág. 11, 14, 25

Antoniadis et al. (1994) A Antoniadis, G Gregoire e IW McKeague. Wavelet methods for curve estimation. Journal of the American Statistical Association, 89(428):1340-1353. Citado na pág. 2, 5

Antoniadis et al. (1999) Anestis Antoniadis, Gérard Grégoire e Guy Nason. Density and hazard rate estimation for right-censored data by using wavelet methods. Journal of the Royal Statistical Society B, 61:63-84. Citado na pág. 2, 5

Bachmann et al. (2000) George Bachmann, Lawrence Narici e Edward Beckenstein. Fourier and wavelet analysis. Springer, New York. Citado na pág. 13

Breslow (1972) Norman E. Breslow. Contribution to the discussion of the paper by D.R. Cox. Journal of the Royal Statistical Society B, 34:216-217. Citado na pág. 2, 11, 16

Cai e Brown (1998) T. Tony Cai e Lawrence D. Brown. Wavelet shrinkage for nonequispaced samples. The Annals of Statistics, 26:1783-1799. Citado na pág. 7

Cai e Sun (2003) Zongwu Cai e Yanqing Sun. Local linear estimation for time-dependent coefficients in Cox’s regression models. Scandinavian Journal of Statistics, 30:93-111. Citado na pág. 1

Campos Filho (2007) Frederico Ferreira Campos Filho. Algoritmos numéricos. LTC, Rio de Janeiro. Citado na pág. 12

Chesneau et al. (2013) Christophe Chesneau, Isha Dewan e Hassan Doosti. Nonparametric estimation of a two dimensional continuous discrete density function by wavelets. Statistical Methodology, 18:64-78. Citado na pág. 2, 5

Cox e Oakes (1984) David R. Cox e David Oakes. Analysis of survival data, volume 21. Chapman \& Hall, New York. Citado na pág. 10

Cox (1972) D.R. Cox. Regression models and life-tables (with discussion). Journal of the Royal Statistical Society B, 34:187-220. Citado na pág. 1, 2, 9, 10, 11, 16

Cox (1975) D.R. Cox. Partial likelihood. Biometrika, 62:269-276. Citado na pág. 10, 11

Cutillo et al. (2013) Luisa Cutillo, Italia De Feis, Christina Nikolaidou e Theofanis Sapatinas. Wavelet density estimation for weighted data. Journal of Statistical Planning and Inference, 146:1-19. Citado na pág. 2,5 
Daubechies (1992) Ingrid Daubechies. Ten lectures on wavelets. Regional Conference Series in Applied Mathematics. Philadelphia: Society for Industrial and Applied Mathematics. Citado na pág. 5, 7

Davison e Hinkley (1997) A.C. Davison e D.V. Hinkley. Bootstrap Methods and Their Application. Cambridge University Press, Cambridge. Citado na pág. 28

Donoho (1993) David L. Donoho. Nonlinear wavelet methods for recovery of signals, densities, and spectra from indirect and noisy data. Em Proceedings of Symposia in Applied Mathematics, volume 47, páginas 173-205. Providence: American Mathematical Society. Citado na pág. 2, 5

Donoho e Johnstone (1995) David L. Donoho e Iain M. Johnstone. Adapting to unknown smoothness via wavelet shrinkage. Journal of the American Statistical Association, 90:1200-1224. Citado na pág. 2, 5

Donoho et al. (1995) David L. Donoho, Iain M. Johnstone, Gérard Kerkyacharian e Dominique Picard. Wavelet shrinkage: asymptopia? Journal of the Royal Statistical Society B, 57:301-369. Citado na pág. 2, 5

Donoho et al. (1998) David L. Donoho, Iain M. Johnstone et al. Minimax estimation via wavelet shrinkage. The Annals of Statistics, 26:879-921. Citado na pág. 2, 5

Efron e Tibshirani (1993) B. Efron e R.J. Tibshirani. An Introduction to the Bootstrap. Chapman \& Hall, New York. Citado na pág. 28

Efron (1977) Bradley Efron. The efficiency of Cox's likelihood function for censored data. Journal of the American Statistical Association, 72:557-565. Citado na pág. 11

Farewel e Prentice (1980) V.T. Farewel e Ross L. Prentice. The approximation of partial likelihood with emphasis on case-control studies. Biometrika, 67:273-278. Citado na pág. 11

Fleming e Harrington (1991) Thomas R. Fleming e David P. Harrington. Counting processes and survival analysis. Wiley, New York. Citado na pág. 49

Gamerman (1991) Dani Gamerman. Dynamic Bayesian models for survival data. Applied Statistics, 40: 63-79. Citado na pág. 1

Gamerman e Lopes (2006) Dani Gamerman e Hedibert F. Lopes. Markov chain Monte Carlo: stochastic simulation for Bayesian inference. CRC Press. Citado na pág. 12, 18

Grambsch e Therneau (1994) Patricia M. Grambsch e Terry M. Therneau. Proportional hazards tests and diagnostics based on weighted residuals. Biometrika, 81:515-526. Citado na pág. 1, 49, 50

Gray (1992) Robert J. Gray. Flexible methods for analyzing survival data using splines, with applications to breast cancer prognosis. Journal of the American Statistical Association, 87:942-951. Citado na pág. 1, 16

Gray (1994) Robert J. Gray. Spline-based tests in survival analysis. Biometrics, 50:640-652. Citado na pág. 16

Haar (1910) Alfred Haar. Zur theorie der orthogonalen funktionensysteme. Mathematische Annalen, 69: 331-371. Citado na pág. 6

Hall e Patil (1995a) Peter Hall e Prakash Patil. Formulae for mean integrated squared error of nonlinear wavelet-based density estimators. The Annals of Statistics, 23:905-928. Citado na pág. 2, 5

Hall e Patil (1995b) Peter Hall e Prakash Patil. On wavelet methods for estimating smooth functions. Bernoulli, 1:41-58. Citado na pág. 2, 5

Hall e Patil (1996) Peter Hall e Prakash Patil. On the choice of smoothing parameter, threshold and truncation in nonparametric regression by non-linear wavelet methods. Journal of the Royal Statistical Society $B, 58: 361-377$. Citado na pág. 2,5 
Hastie e Tibshirani (1993) Trevor Hastie e Robert Tibshirani. Varying-coefficient models (with discussion). Journal of the Royal Statistical Society B, 55:757-796. Citado na pág. 1

Hess (1994) Kenneth R. Hess. Assessing time-by-covariate interactions in proportional hazards regression models using cubic spline functions. Statistics in Medicine, 13:1045-1062. Citado na pág. 1

Härdle et al. (1998) Wolfgang Härdle, Gèrard Kerkyacharian e Dominique Picard. Wavelets, approximation, and statistical applications. Lecture Notes in Statistics. Springer, New York. Citado na pág. 5

Huang e Liu (2006) Jianhua Z. Huang e Linxu Liu. Polynomial spline estimation and inference of proportional hazards regression models with flexible relative risk form. Biometrics, 62:793-802. Citado na pág. 16

Huang e Shen (2004) Jianhua Z. Huang e Haipeng Shen. Functional coefficient regression models for non-linear time series: A polynomial spline approach. Scandinavian Journal of Statistics, 31:515-534. Citado na pág. 45

Kalbfleisch e Prentice (1973) John D. Kalbfleisch e Ross L. Prentice. Marginal likelihoods based on Cox's regression and life model. Biometrika, 60:267-278. Citado na pág. 2, 16

Kerkyacharian e Picard (2004) Gérard Kerkyacharian e Dominique Picard. Regression in random design and warped wavelets. Bernoulli, 10:1053-1105. Citado na pág. 7

Klein e Moeschberger (2003) John P. Klein e Melvin L. Moeschberger. Survival analysis: Techniques for censored and truncated data. Springer, New York. Citado na pág. 12, 16, 17

Lin et al. (2006) Jiang Lin, Daowen Zhang e Marie Davidian. Smoothing spline-based score tests for proportional hazards models. Biometrics, 62:803-812. Citado na pág. 1, 16

Mallat (2008) Stephane Mallat. A wavelet tour of signal processing: The sparse way (3rd ed.). Academic press, New York. Citado na pág. 5

Martinussen et al. (2002) Torben Martinussen, Thomas H. Scheike e Ib. M. Skovgaard. Efficient estimation of fixed and time-varying covariate effects in multiplicative intensity models. Scandinavian Journal of Statistics, 29:57-74. Citado na pág. 1

Marzec e Marzec (1997) Leszek Marzec e Pawel Marzec. On fitting Cox's regression model with timedependent coefficients. Biometrika, 84:901-908. Citado na pág. 1

Morettin (1999) Pedro A. Morettin. Ondas e ondaletas: Da análise de Fourier à análise de ondaletas. Edusp, São Paulo. Citado na pág. 5, 13

Morettin e Toloi (2006) Pedro A. Morettin e Clélia Toloi. Análise de séries temporais. Edgard Blücher, São Paulo. Citado na pág. 13

Murphy (1993) Susan A. Murphy. Testing for a time dependent coefficient in Cox's regression model. Scandinavian Journal of Statistics, 20:35-50. Citado na pág. 1

Murphy e Sen (1991) Susan Allbritton Murphy e Pranab K. Sen. Time-dependent coefficients in a Cox-type regression model. Stochastic Processes and their Applications, 39:153-180. Citado na pág. 1

Nason (2008) G.P. Nason. Wavelet Methods in Statistics with R. Springer. Citado na pág. 5

Nason e Silverman (1994) Guy P. Nason e Bernard W. Silverman. The discrete wavelet transform in S. Journal of Computational and Graphical Statistics, 3:163-191. Citado na pág. 2, 5

Pedroso-de Lima e Sen (1999) Antonio C. Pedroso-de Lima e Pranab K. Sen. Time-dependent coefficients in a multi-event model for survival analysis. Journal of Statistical Planning and Inference, 75:393-414. Citado na pág. 1 
Peto (1972) R. Peto. Contribuição à discussão do artigo de DR Cox. Journal of the Real Statistics Society $B, 34: 205-207$. Citado na pág. 11

Pettitt e Bin Daud (1990) A.N. Pettitt e I. Bin Daud. Investigating time dependence in Cox's proportional hazards model. Applied Statistics, 39:313-329. Citado na pág. 1

R Core Team (2014) R Core Team. R: A Language and Environment for Statistical Computing. R Foundation for Statistical Computing, Vienna, Austria, 2014. Citado na pág. 35, 66

Ruggiero e Lopes (1997) Márcia A. Gomes Ruggiero e Vera Lúcia da Rocha Lopes. Cálculo numérico: aspectos teóricos e computacionais. Pearson, São Paulo. Citado na pág. 70

Saegusa et al. (2014) Takumi Saegusa, Chongzhi Di e Ying Qing Chen. Hypothesis testing for an extended Cox model with time-varying coefficients. Biometrics, 70:619-628. Citado na pág. 1, 16

Sargent (1997) Daniel J. Sargent. A flexible approach to time-varying coefficients in the Cox regression setting. Lifetime Data Analysis, 3:13-25. Citado na pág. 1

Schoenfeld (1982) David Schoenfeld. Partial residuals for the proportional hazards regression model. Biometrika, 69:239-241. Citado na pág. 1, 11

Schwarz (1978) Gideon Schwarz. Estimating the dimension of a model. The Annals of Statistics, 6:461464. Citado na pág. 45

Sen et al. (2010) Pranab K. Sen, Julio M. Singer e Antonio C. Pedroso-de Lima. From finite sample to asymptotic methods in statistics. Cambridge University Press, New York. Citado na pág. 16

Shumway e Stoffer (2010) Robert H. Shumway e David S. Stoffer. Time series analysis and its applications. Springer, New York. Citado na pág. 6

Sleeper e Harrington (1990) Lynn A. Sleeper e David P. Harrington. Regression splines in the Cox model with application to covariate effects in liver disease. Journal of the American Statistical Association, 85: 941-949. Citado na pág. 16, 45

Tian et al. (2005) Lu Tian, David Zucker e L.J. Wei. On the Cox model with time-varying regression coefficients. Journal of the American Statistical Association, 100:172-183. Citado na pág. 1, 17

Tsiatis (1981) Anastasios A. Tsiatis. A large sample study of Cox's regression model. The Annals of Statistics, 9:93-108. Citado na pág. 11

Vidakovic (1999) Brani Vidakovic. Statistical modeling by wavelets. Wiley, New York. Citado na pág. 5, 13

Winnett e Sasieni (2003) Angela Winnett e Peter Sasieni. Iterated residuals and time-varying covariate effects in Cox regression. Journal of the Royal Statistical Society B, 65:473-488. Citado na pág. 1

Zucker e Karr (1990) David M. Zucker e Alan F. Karr. Nonparametric survival analysis with timedependent covariate effects: a penalized partial likelihood approach. The Annals of Statistics, 18:329-353. Citado na pág. 1 